\title{
A DIAGRAM ALGEBRA FOR SOERGEL MODULES CORRESPONDING TO SMOOTH SCHUBERT VARIETIES
}

\begin{abstract}
ANTONIO SARTORI
ABSTRACT. Using combinatorial properties of symmetric polynomials, we compute explicitly the Soergel modules for some permutations whose corresponding Schubert varieties are rationally smooth. We build from them diagram algebras whose module categories are equivalent to the subquotient categories of the BGG category $\mathcal{O}\left(\mathfrak{g l}_{n}\right)$ which show up in categorification of $\mathfrak{g l}(1 \mid 1)$ representations. We construct diagrammatically the graded cellular structure and the properly stratified structure of these algebras.
\end{abstract}

\section{Contents}

1. Introduction

2. Symmetric polynomials

3. Some canonical bases elements

4. Soergel modules

5. The diagram algebra

6. Category $\mathcal{O}$

Acknowledgments

References

\section{INTRODUCTION}

The BGG category $\mathcal{O}(\mathfrak{g})$, introduced in BGG76, is a fundamental tool for studying the representation theory of a reductive Lie algebra $\mathfrak{g}$. Its combinatorial properties are quite surprising: the Kazhdan-Lusztig conjecture, for example, relates decomposition numbers in the category $\mathcal{O}(\mathfrak{g})$ with certain polynomials which appear naturally in the theory of Hecke algebras KL79.

The explicit structure of this category remained obscure until the fundamental work of Soergel Soe90, who constructed a fully faithful functor $\mathbb{V}$ from the additive category of projective objects of $\mathcal{O}(\mathfrak{g})$ to a category of modules (which we call Soergel modules) over a polynomial ring. In principle, this gives a way to do explicit computations in the category $\mathcal{O}(\mathfrak{g})$ (see Str03] for some examples in small cases); however, determining Soergel modules and their homomorphisms is not affordable in general. In this paper, we present such a description for some subquotient categories of $\mathcal{O}_{0}=\mathcal{O}_{0}\left(\mathfrak{g l}_{n}\right)$, which are denoted by $\mathcal{Q}_{k}(\mathfrak{m})$ in Sar13. These are a

Received by the editors October 14, 2013 and, in revised form, December 4, 2013.

2010 Mathematics Subject Classification. Primary 16W50; Secondary 13F20, 05E05, 17B10.

Key words and phrases. Diagram algebra, symmetric polynomials, category $\mathcal{O}$, Soergel modules, Khovanov algebra.

This work has been supported by the Graduiertenkolleg 1150, funded by the Deutsche Forschungsgemeinschaft. 
particular case of the "generalized parabolic subcategories" introduced in [FKM02] and developed further in Maz04. Our interest for these particular categories is motivated by the fact that they can be used to categorify tensor powers of the vector representation of $\mathfrak{g l}(1 \mid 1)$ (see [Sar13]).

The central role played by category $\mathcal{O}$ in categorification makes the ability to do explicit computations crucial. Khovanov homology Kho00, which is ultimately an $\mathfrak{s l}_{2}$-categorification through category $\mathcal{O}$, has proven very useful in its application to low dimensional topology, because of its explicit description. A diagrammatic approach to understand some parabolic subcategories of the category $\mathcal{O}\left(\mathfrak{g l}_{n}\right)$ has been successfully carried out by Brundan and Stroppel: in a series of four papers ( $\mathrm{BS} 11 \mathrm{a}], \mathrm{BS10}$, , BS11b], BS12b]) they define diagrammatic algebras (called generalized Khovanov algebras) which are isomorphic to the endomorphism rings of projective generators of the parabolic category $\mathcal{O}^{\mathfrak{p}}\left(\mathfrak{g l}_{n}\right)$, where $\mathfrak{p} \subseteq \mathfrak{g l}_{n}$ is a maximal parabolic subalgebra. They are able to interpret diagrammatically translation functors, and hence compute explicitly homomorphism spaces between them. Using the machinery they have constructed, they can connect the category $\mathcal{O}\left(\mathfrak{g l}_{n}\right)$ to the category of finite dimensional representations of the super Lie algebra $\mathfrak{g l}(r \mid s)$; they also find interesting connections to representations of the walled Brauer algebra BS12a.

The goal of this paper is to develop a similar approach in the case of the categories $Q_{k}(m)$. Our main result is a diagrammatic/combinatorial definition of graded algebras $A_{n, k}$ whose module categories are equivalent to $Q_{k}(\mathrm{~m})$.

The main tools of our construction are Soergel modules and combinatorics of symmetric polynomials. Soergel modules for the symmetric group $\mathbb{S}_{n}$ (or for $\mathfrak{g l}_{n}$ ) are modules $\mathrm{C}_{z}$ over the polynomial ring in $n$ variables $R=\mathbb{C}\left[x_{1}, \ldots, x_{n}\right]$ and are labeled by permutations $z \in \mathbb{S}_{n}$. By definition, they are the indecomposable direct summands of modules of the type

$$
B \otimes_{B^{s_{i}}} B \otimes \cdots \otimes B \otimes_{B^{s_{i}}} B \otimes_{B} \mathbb{C}
$$

where $B=R /\left(R_{+}\right)^{\mathbb{S}_{n}} R$ is the ring of the coinvariants and $B^{s}$ are the invariants under a simple reflection $s \in \mathbb{S}_{n}$. In particular, if we choose a reduced expression $s_{i_{r}} \cdots s_{i_{1}}$ for $z$, then $\mathrm{C}_{z}$ is the indecomposable direct summand of (1.1) containing $1 \otimes \cdots \otimes 1$.

Although they could seem apparently harmless, identifying explicitly the direct summands in (1.1) is quite tricky, even in some small examples. Describing homomorphism spaces between such summands is in general hard. A significant simplification occurs when the Soergel module $C_{z}$ happens to be cyclic: in this case it is enough to determine the annihilator of $\mathrm{C}_{z}$, which is the same as the annihilator of (1.1). Moreover, in order to describe the homomorphisms between two cyclic $R$-modules it is enough to study the quotient ideal between the corresponding annihilators.

The condition of a Soergel module being cyclic arises naturally since it is equivalent to the rational smoothness of the corresponding Schubert variety in the full flag variety (cf. [KL79, Appendix]); an easy combinatorial criterion for determining which Schubert varieties are rationally smooth is given in GR02. It is quite surprising that all the Soergel modules needed for understanding the categories $Q_{k}(n)$ satisfy this condition.

We point out that some work has been done to understand Soergel bimodules Soe92, which are the equivariant version of Soergel modules and are obtained by 
dropping the last $\otimes_{B} \mathbb{C}$ in (1.1) (see [Wil11, [EK09]; we would also like to mention that Soergel bimodules have been proved useful for the recent algebraic proof [EW12] of the Kazhdan-Lusztig conjecture). On the other side, as far as we know, this is the first attempt to describe explicitly Soergel modules. We believe that Soergel modules are in some sense more difficult than Soergel bimodules because of the lack of symmetry of (1.1).

Despite the deep background, the computations required throughout the paper are elementary, although quite involved. In order to keep the paper readable also for non-experts, we decided to start the paper with combinatorial properties of symmetric polynomials and postpone the introduction of category $\mathcal{O}$ after the definition of the diagram algebra.

Overview of the paper. Let us now explain in more detail the structure and the results of the paper. We start in Section 2 by studying quotients of the polynomial ring $R$ modulo ideals $I_{\boldsymbol{b}}$ generated by some complete symmetric polynomials in a subset of the variables. More precisely, if $\boldsymbol{b}=\left(b_{1}, \ldots, b_{n}\right)$ is a non-increasing sequence of positive integers, then we let

$$
I_{\boldsymbol{b}}=\left(h_{b_{1}}\left(x_{1}\right), h_{b_{2}}\left(x_{1}, x_{2}\right), \ldots, h_{b_{n}}\left(x_{1}, \ldots, x_{n}\right)\right),
$$

where the $h_{i}$ 's are complete symmetric polynomials of degree $i$ in the variables indicated. This definition will prove very helpful to describe the Soergel modules we are interested in. To study such ideals, we will use the powerful machinery of Groebner bases, which we will briefly review.

In Section 3 we introduce the Hecke algebra and define the Kazhdan-Lusztig polynomials. Then we compute explicitly some Kazhdan-Lusztig basis elements, which we will use later to determine the dimension of the corresponding Soergel modules.

In Section 4 we briefly recall the definition of Soergel modules and then compute explicitly some of them. In particular, we compute all Soergel modules $\mathrm{C}_{w_{k} z}$ where $z$ is in the set $D$ of shortest coset representative for $\left(\mathbb{S}_{k} \times \mathbb{S}_{n-k}\right) \backslash \mathbb{S}_{n}$ and $w_{k}$ is the longest element of $\mathbb{S}_{k}$. Our strategy is the following: first, we show that the Soergel module $\mathrm{C}_{w_{k} z}$ is cyclic. Then for every such $z$ we choose a clever reduced expression $s_{i_{r}} \cdots s_{i_{1}}$ and we determine some partial symmetric polynomials which lie in the annihilator of the module (1.1). We deduce that they are enough to generate the whole annihilator by comparing the dimensions, using the results we collected in the previous sections. Our first main result is:

Theorem 1.1 (see Theorem 4.10). For each $z \in D$ there is an associated $\boldsymbol{b}$ sequence $\boldsymbol{b}^{z}$ such that the Soergel module $C_{w_{k} z}$ is isomorphic to $R / I_{\boldsymbol{b}^{z}}$.

Our next main result is the explicit description of the spaces of homomorphisms between these Soergel modules modulo the morphisms which factor through some "wrong" Soergel modules (see Theorem 4.17). This is motivated by the fact that the categories $Q_{k}(\mathrm{~m})$ are subquotient categories of $\mathcal{O}_{0}$; hence they are described by algebras which are the endomorphism rings of some projective modules of $\mathcal{O}_{0}$ modulo morphisms which factor through some "wrong" projective modules (see Section 6 for the details).

To make the statement of Theorem 4.17 clear and usable, in Section 5 we introduce diagrams which describe the corresponding homomorphism spaces. Putting together all the homomorphism spaces and translating into our diagram language 
we obtain diagram algebras $A_{n, k}$. These algebras resemble the generalized Khovanov algebras of [BS11a. Recall that the generalized Khovanov algebras categorify tensor powers of $\mathfrak{s l}_{2}$-representations, and the diagrams which build these algebras are the same diagrams of [FK97] describing $\mathfrak{s l}_{2}$-intertwiners. Unsurprisingly, the same happens for our algebras $A_{n, k}$ : the diagrams which we use here are essentially the same diagrams which we introduce in the related paper Sar13 for describing $\mathfrak{g l}(1 \mid 1)$-intertwiners, and indeed the resulting algebras categorify tensor powers of $\mathfrak{g l}(1 \mid 1)$-representations.

We remark that the algebras $A_{n, k}$ are graded, the grading coming from the one on polynomial rings. Since the algebras $A_{n, k}$ describe categories which come from category $\mathcal{O}$, we could deduce from abstract Lie theory that they enjoy very nice properties, such as cellularity [GL96] and proper stratifiedness [FKM02] (which is a generalization of quasi-hereditariness). In the second part of Section 5 we show how these properties can be proved independently by constructing explicitly cellular, standard and projective modules using our diagrams, and we obtain:

Theorem 1.2 (see Proposition 5.18 and Theorem 5.24). For all $n \in \mathbb{N}, 0 \leq k \leq n$ the algebra $A_{n, k}$ is graded cellular and properly stratified.

In Section 6 we finally explain in detail the relation with category $\mathcal{O}$ which underlies all of the paper. We recall from [Sar13] the definition of the categories $Q_{k}(\mathrm{~m})$ and we state our main result:

Theorem 1.3 (see Theorem 6.7). The category $\mathcal{Q}_{k}(\mathrm{~m})$ from Sar13 is equivalent to $\operatorname{gmod}-A_{n, k}$.

This gives an explicit description of the categories $Q_{k}(\mathfrak{m})$ categorifying $\mathfrak{g l}(1 \mid 1)$ and makes it possible to compute examples of that categorification. One of the main applications is the computation of the endomorphism rings of the functors $\mathcal{E}_{k}$ and $\mathcal{F}_{k}$ from Sar13. (see Theorem 6.11).

We remark that there is a connection between the algebra $A_{n, k}$ and the cohomology of closed attracting varieties in the Springer fiber of hook type sitting inside the full flag variety; see Sar14]. In particular, we conjecture that it is possible to construct a convolution product on these cohomology rings as in SW12] so that one can recover the full algebra $A_{n, k}$ using geometry.

\section{Symmetric POLYNOMials}

In this section we are going to study some rings obtained as quotients of a polynomial ring modulo an ideal generated by complete symmetric functions in some subsets of variables. We start recalling some easy standard facts about symmetric polynomials.

We let $R=\mathbb{C}\left[x_{1}, \ldots, x_{n}\right]$ be a polynomial ring. We consider it as a graded ring with $\operatorname{deg} x_{i}=2$ for every $i$.

2.1. Complete symmetric polynomials. The complete symmetric polynomials are defined as

$$
h_{j}\left(x_{1}, \ldots, x_{n}\right)=\sum_{1 \leq i_{1} \leq \cdots \leq i_{j} \leq n} x_{i_{1}} \cdots x_{i_{j}}
$$

for every $j \geq 1$ so that for example $h_{2}\left(x_{1}, x_{2}\right)=x_{1}^{2}+x_{1} x_{2}+x_{2}^{2}$. We also set $h_{0}\left(x_{1}, \ldots, x_{n}\right)=1$, while if $n=0$ (i.e., we have zero variables), we let $h_{i}()=0$ for 
every $i \geq 1$. The symmetric group $\mathbb{S}_{n}$ acts on $R$ permuting the variables, and the polynomials $h_{i}\left(x_{1}, \ldots, x_{n}\right)$ are invariant under this action; in fact, they generate the whole algebra $R^{\mathbb{S}^{n}}$ of invariant polynomials (see [Ful97, Section 6]).

We will consider complete symmetric polynomials in some subset of the variables of $R$. The following formula helps us to decompose complete symmetric polynomials in $k$ variables as complete symmetric polynomials in $\ell$ and $k-\ell$ variables, for every $\ell=1, \ldots, k-1$ :

$$
h_{j}\left(x_{1}, \ldots, x_{k}\right)=\sum_{n=0}^{j} h_{n}\left(x_{1}, \ldots, x_{\ell}\right) h_{j-n}\left(x_{\ell+1}, \ldots, x_{k}\right) .
$$

Another formula allows us to express complete symmetric polynomials in $k-1$ variables in terms of complete symmetric polynomials in $k$ variables:

$$
h_{j}\left(x_{1}, \ldots, x_{k-1}\right)=h_{j}\left(x_{1}, \ldots, x_{k}\right)-x_{k} h_{j-1}\left(x_{1}, \ldots, x_{k}\right) .
$$

Both (2.2) and (2.3) can be checked easily by comparing which monomials appear on both sides.

For $1 \leq i \leq n-1$ let $R^{s_{i}}$ be the subring of $R$ consisting of polynomials invariant under the simple transposition $s_{i}$. We recall from Dem73 the definition of the classical Demazure operator $\partial_{i}: R \rightarrow R^{s_{i}}\langle 2\rangle$, given by

$$
\partial_{i}: f \longmapsto \frac{f-s_{i}(f)}{x_{i}-x_{i+1}}
$$

The operator $\partial_{i}$ is linear, vanishes on $R^{s_{i}}$ and satisfies $\partial_{i}(f g)=f \partial_{i} g$ whenever $f \in R^{s_{i}}$. Also let $P_{i}: R \rightarrow R$ be defined by $P_{i}(f)=f-x_{i} \partial_{i}(f)$. It is easy to show that $P_{i}$ also has values in $R^{s_{i}}$. The operators $\partial_{i}$ and $P_{i}$ can be used to define the decomposition $R \cong R^{s_{i}} \oplus x_{i} R^{s_{i}}$ as an $R^{s_{i}}$-module by

$$
f \mapsto P_{i} f \oplus x_{i} \partial_{i} f .
$$

Demazure operators have the nice property of sending complete symmetric polynomials to other complete symmetric polynomials:

Lemma 2.1. For all $j \geq 1$ we have

$$
\partial_{k} h_{j}\left(x_{1}, \ldots, x_{k}\right)=h_{j-1}\left(x_{1}, x_{2}, \ldots, x_{k+1}\right) .
$$

We omit the proof, which is a straightforward computation.

2.2. Ideals generated by complete symmetric polynomials. We are going to study quotients rings of $R$ generated by some of the $h_{i}$ 's. Let

$$
\mathscr{B}^{\prime}=\left\{\boldsymbol{b}=\left(b_{1}, \ldots, b_{n}\right) \in \mathbb{N}^{n} \mid b_{i} \geq b_{i+1} \geq b_{i}-1\right\} .
$$

In other words, $\mathscr{B}^{\prime}$ is the set of weakly decreasing sequences of positive numbers such that the difference between two consecutive items is at most one. For every sequence $\boldsymbol{b} \in \mathscr{B}^{\prime}$ let $I_{\boldsymbol{b}} \subset R$ be the ideal generated by

$$
h_{b_{1}}\left(x_{1}\right), h_{b_{2}}\left(x_{1}, x_{2}\right), \ldots, h_{b_{n}}\left(x_{1}, \ldots, x_{n}\right) .
$$

Set also $R_{\boldsymbol{b}}=R / I_{\boldsymbol{b}}$.

We will shortly recall the definition of Groebner bases, which are a useful tool for studying ideals in polynomial rings; for a complete reference see CLO07, Chapter 2]. Let us fix a lexicographic monomial order on $R$ with

$$
x_{n}>x_{n-1}>\cdots>x_{1} .
$$


With respect to this ordering, each polynomial $p \in R$ has a leading term $\operatorname{LT}(p)$. Given an ideal $I \subseteq R$, let $\operatorname{LT}(I)=\{\operatorname{LT}(p) \mid p \in I\}$ be the set of leading terms of elements of $I$ and let $\langle\operatorname{LT}(I)\rangle$ be the ideal they generate. We recall that a finite subset $\left\{p_{1}, \ldots, p_{r}\right\}$ of an ideal $I$ of $R$ is called a Groebner basis if the leading terms of the $p_{1}, \ldots, p_{r}$ generate $\langle\operatorname{LT}(I)\rangle$. Then we have:

Lemma 2.2. The polynomials (2.8) are a Groebner basis for $I_{\boldsymbol{b}}$ with respect to the order (2.9).

Proof. By [CLO07, Theorem 2.9.3 and Proposition 2.9.4] it is enough to check that the leading monomials of the polynomials (2.8) are pairwise relatively prime. This is obvious.

Proposition 2.3. Let $\boldsymbol{b} \in \mathscr{B}^{\prime}$. The quotient ring $R_{\boldsymbol{b}}=R / I_{\boldsymbol{b}}$ has dimension $b_{1} \cdots b_{n}$, and $a \mathbb{C}$-basis is given by

$$
\left\{\boldsymbol{x}^{j}=x_{1}^{j_{1}} \cdots x_{n}^{j_{n}} \mid 0 \leq j_{i}<b_{i}\right\} .
$$

Proof. By the theory of Groebner bases (cf. [CLO07, Proposition 2.6.1]), any $f \in R$ can be written uniquely as $f=g+r$, with $g \in I_{b}$ and $r$ such that no term of $r$ is divisible by any of the leading terms of the Groebner basis (2.8); that is, $r$ is a linear combination of the monomials (2.10). This means exactly that the monomials (2.10) are a basis of $R_{b}$.

Example 2.4. Let $\boldsymbol{b}=(1, \ldots, 1)$. Then $x_{i}=h_{1}\left(x_{1}, \ldots, x_{i}\right)-h_{1}\left(x_{1}, \ldots, x_{i-1}\right)$ lies in $I_{\boldsymbol{b}}$ for each $i$; hence $I_{\boldsymbol{b}}=\left(x_{1}, \ldots, x_{n}\right)$ and $R_{\boldsymbol{b}} \cong \mathbb{C}$ is one dimensional.

Example 2.5. Let $\boldsymbol{b}=(n, n-1, \ldots, 1)$. Then it is easy to show that the ideal $I_{\boldsymbol{b}}$ is the ideal generated by the symmetric polynomials in $n$ variables with zero constant term and $R_{\boldsymbol{b}}$ is the ring of the coinvariants $R /\left(R_{+}^{\mathbb{S}_{n}}\right)$, isomorphic to the cohomology of the full flag variety of $\mathbb{C}^{n}$ (see [Ful97, §10.2, Proposition 3]). As given by Proposition 2.3, it has dimension $n$ ! and it is well-known that a monomial basis is given by

$$
\left\{x_{1}^{j_{1}} \cdots x_{n}^{j_{n}} \mid 0 \leq j_{i} \leq n-i\right\} .
$$

2.3. Morphisms between quotient rings. Next, we are going to determine all $R$-module homomorphisms between rings $R_{\boldsymbol{b}}$.

Proposition 2.6. Let $\boldsymbol{b}, \boldsymbol{b}^{\prime} \in \mathscr{B}^{\prime}$, and let $c_{i}=\max \left\{b_{i}^{\prime}-b_{i}, 0\right\}$. Then a $\mathbb{C}$-basis of $\operatorname{Hom}_{R}\left(R_{\boldsymbol{b}}, R_{\boldsymbol{b}^{\prime}}\right)$ is given by

$$
\left\{1 \mapsto x_{1}^{j_{1}} \cdots x_{n}^{j_{n}} \mid c_{i} \leq j_{i}<b_{i}^{\prime}\right\} .
$$

The proof consists of several lemmas.

Lemma 2.7. Let $\boldsymbol{b} \in \mathscr{B}^{\prime}$. Then $h_{a}\left(x_{1}, \ldots, x_{i}\right) \in I_{\boldsymbol{b}}$ for every $a \geq b_{i}$.

Proof. We prove by induction on $\ell \geq 0$ that $h_{b_{i}+\ell}\left(x_{1}, \ldots, x_{i}\right) \in I_{\boldsymbol{b}}$ for every $i=$ $1, \ldots, n$. For $\ell=0$ the statement follows from the definition. For the inductive step, choose an index $i$ and pick $j<i$ maximal such that $b_{j}=b_{i}+1$ (or let $j=0$ if such an index does not exist) and write using iteratively (2.3):

$$
\begin{aligned}
h_{b_{i}+\ell}\left(x_{1}, \ldots, x_{i}\right)= & h_{b_{i}+\ell}\left(x_{1}, \ldots, x_{j}\right)+x_{j+1} h_{b_{i}+\ell-1}\left(x_{1}, \ldots, x_{j+1}\right) \\
& +\cdots+x_{i-1} h_{b_{i}+\ell-1}\left(x_{1}, \ldots, x_{i-1}\right)+x_{i} h_{b_{i}+\ell-1}\left(x_{1}, \ldots, x_{i}\right) .
\end{aligned}
$$

Since $b_{i}+\ell=b_{j}+\ell-1$, the terms on the right all lie in $I_{\boldsymbol{b}}$ by the inductive hypothesis. 
Lemma 2.8. Let $\boldsymbol{b}=\left(b_{1}, \ldots, b_{n}\right) \in \mathscr{B}^{\prime}$ and

$$
\boldsymbol{b}^{\prime}=\left(b_{1}, \ldots, b_{i-1}, b_{i}+1, b_{i+1}, \ldots, b_{n}\right)
$$

for some $i$. Suppose that also $\boldsymbol{b}^{\prime} \in \mathscr{B}^{\prime}$. Then $I_{\boldsymbol{b}^{\prime}} \subset I_{\boldsymbol{b}}$ while $x_{i} I_{\boldsymbol{b}} \subseteq I_{\boldsymbol{b}^{\prime}}$.

Proof. It follows directly from Lemma 2.7 that $I_{\boldsymbol{b}^{\prime}} \subset I_{\boldsymbol{b}}$. For the other assertion, since $h_{b_{j}}\left(x_{1}, \ldots, x_{j}\right) \in I_{\boldsymbol{b}^{\prime}}$ for every $j \neq i$, we only need to prove that $x_{i} h_{b_{i}}\left(x_{1}, \ldots, x_{i}\right) \in I_{\boldsymbol{b}^{\prime}}$. By (2.3) we have

$$
x_{i} h_{b_{i}}\left(x_{1}, \ldots, x_{i}\right)=h_{b_{i}+1}\left(x_{1}, \ldots, x_{i}\right)-h_{b_{i}+1}\left(x_{1}, \ldots, x_{i-1}\right) .
$$

Since we suppose $\boldsymbol{b}^{\prime} \in \mathscr{B}^{\prime}$, it follows that $b_{i-1}=b_{i}+1$; hence the r.h.s. of (2.14) lies in $I_{b^{\prime}}$.

We will call two sequences $\boldsymbol{b}, \boldsymbol{b}^{\prime} \in \mathscr{B}^{\prime}$ that satisfy the hypothesis of Lemma 2.8 (without regarding the order) near each other.

Lemma 2.9. Let $\boldsymbol{b}, \boldsymbol{b}^{\prime} \in \mathscr{B}^{\prime}$ and set $c_{i}=\max \left\{b_{i}^{\prime}-b_{i}, 0\right\}$. Then $x_{1}^{c_{1}} \cdots x_{n}^{c_{n}} I_{\boldsymbol{b}} \subseteq I_{\boldsymbol{b}^{\prime}}$.

Proof. We can find a sequence $\boldsymbol{b}=\boldsymbol{b}^{(0)}, \boldsymbol{b}^{(1)}, \ldots, \boldsymbol{b}^{(N)}=\boldsymbol{b}^{\prime}$ with $\boldsymbol{b}^{(k)} \in \mathscr{B}^{\prime}$ for each $k$ and $N=\sum_{i}\left|b_{i}-b_{i}^{\prime}\right|$ such that $\boldsymbol{b}^{(i)}$ and $\boldsymbol{b}^{(i+1)}$ are near each other. Then the claim follows applying iteratively Lemma 2.8 .

Lemma 2.10. Let $\boldsymbol{b}, \boldsymbol{b}^{\prime} \in \mathscr{B}^{\prime}$. Let $c_{i}=\max \left\{b_{i}^{\prime}-b_{i}, 0\right\}$. Suppose $p \in R$ is such that $p I_{\boldsymbol{b}} \subseteq I_{\boldsymbol{b}^{\prime}}$. Then $x_{1}^{c_{1}} \cdots x_{n}^{c_{n}} \mid p$.

Proof. We prove the claim by induction on the leading term of $p$, using the lexicographic order (2.9). Let $p^{\prime}$ be the leading term of $p$ and pick an index $1 \leq i \leq n$. By assumption, $p h_{b_{i}}\left(x_{1}, \ldots, x_{i}\right) \in I_{\boldsymbol{b}^{\prime}}$. By the theory of Groebner bases, the leading term of $p h_{b_{i}}\left(x_{1}, \ldots, x_{i}\right)$ is divisible by $x_{1}^{b_{1}^{\prime}} \cdots x_{n}^{b_{n}^{\prime}}$, and this leading term is just $p^{\prime} x_{i}^{b_{i}}$. It follows immediately that $x_{1}^{c_{1}} \cdots x_{n}^{c_{n}} \mid p^{\prime}$. By Lemma 2.9 we then know that $p^{\prime} I_{\boldsymbol{b}} \subseteq I_{\boldsymbol{b}^{\prime}}$, hence also $\left(p-p^{\prime}\right) I_{\boldsymbol{b}} \subseteq I_{\boldsymbol{b}^{\prime}}$. By induction, we may assume that $x_{1}^{c_{1}} \cdots x_{n}^{c_{n}} \mid\left(p-p^{\prime}\right)$, and we are done.

Proof of Proposition 2.6. It follows from Lemma 2.9 that the elements of (2.12) indeed define morphisms $R_{\boldsymbol{b}} \rightarrow R_{\boldsymbol{b}^{\prime}}$. By Proposition 2.3 they are linearly independent, and by Lemma 2.10 they are a set of generators.

2.4. Duality. The category of finite dimensional $R$-modules has a duality, given by

$$
M^{*}=\operatorname{Hom}_{\mathbb{C}}(M, \mathbb{C}) .
$$

In fact, the vector space $M^{*}$ is endowed with an $R$-action by setting $(r \cdot f)(m)=$ $f(r \cdot m)$ for all $f \in M^{*}, m \in M, r \in R$ (since $R$ is commutative). If $M$ is graded, the dual inherits a grading declaring $\left(M^{*}\right)_{j}=\left(M_{-j}\right)^{*}$.

Now consider some $\boldsymbol{b} \in \mathscr{B}^{\prime}$. The monomial basis (2.10) of $R_{\boldsymbol{b}}$ has a unique element of maximal degree $b=2\left(b_{1}+\cdots+b_{n}-n\right)$, namely $\boldsymbol{x}^{\boldsymbol{b}-\mathbf{1}}$ where $\mathbf{1}=(1, \ldots, 1)$ and $\boldsymbol{b}-\mathbf{1}$ is the sequence $\left(b_{1}-1, \ldots, b_{n}-1\right)$. We define a symmetric bilinear form $(\cdot, \cdot)$ on $R_{b}$ by letting

$$
\left(\boldsymbol{x}^{\boldsymbol{j}}, \boldsymbol{x}^{\boldsymbol{j}^{\prime}}\right)= \begin{cases}1 & \text { if } \boldsymbol{j}+\boldsymbol{j}^{\prime}=\boldsymbol{b}-\mathbf{1} \\ 0 & \text { otherwise }\end{cases}
$$


on the monomial basis (2.10), where sequences are added termwise. Since this form is clearly non-degenerate, we get an isomorphism of graded $R$-modules:

$$
R_{\boldsymbol{b}} \cong R_{\boldsymbol{b}}^{*}\langle-b\rangle \text { for every } \boldsymbol{b} \in \mathscr{B}^{\prime} .
$$

The degree shift comes out because the bilinear form pairs the degree $i$ component of $R_{\boldsymbol{b}}$ with its component of degree $b-i$.

By the properties of a duality, we have

$$
\operatorname{Hom}_{R}\left(R_{\boldsymbol{b}}, R_{\boldsymbol{b}^{\prime}}\right) \cong \operatorname{Hom}_{R}\left(R_{\boldsymbol{b}^{\prime}}^{*}, R_{\boldsymbol{b}}^{*}\right) \cong \operatorname{Hom}_{R}\left(R_{\boldsymbol{b}^{\prime}}, R_{\boldsymbol{b}}\right)\left\langle b^{\prime}-b\right\rangle
$$

for any $\boldsymbol{b}, \boldsymbol{b}^{\prime} \in \mathscr{B}^{\prime}$. It is not difficult to see that the composite isomorphism is given explicitly by

$$
\Theta:(1 \mapsto p) \longmapsto\left(1 \mapsto \frac{\boldsymbol{x}^{\boldsymbol{b}-1}}{\boldsymbol{x}^{\boldsymbol{b}^{\prime}-1}} p\right)
$$

2.5. Schubert polynomials. We recall some basic facts about Schubert polynomials, referring to Mac91 for more details. Let $w \in \mathbb{S}_{n}$ be a permutation; then the operator $\partial_{w}=\partial_{i_{1}} \cdots \partial_{i_{r}}$, where $w=s_{i_{1}} \cdots s_{i_{r}}$ is some reduced expression, does not depend on the particular chosen reduced expression and is hence well-defined. Let $w_{n} \in \mathbb{S}_{n}$ be the longest element. Then one defines the Schubert polynomial

$$
\mathfrak{S}_{w}\left(x_{1}, \ldots, x_{n}\right)=\partial_{w^{-1} w_{n}} x_{1}^{n-1} x_{2}^{n-2} \cdots x_{n-1}
$$

for each $w \in \mathbb{S}_{n}$. The Schubert polynomials give a basis of $R /\left(R_{+}^{\mathbb{S}_{n}}\right)$. It follows from the definition that $\operatorname{deg} \mathfrak{S}_{w}\left(x_{1}, \ldots, x_{n}\right)=2 \ell(w)$.

For our purposes, it will be more convenient to have a monomial basis of $R /\left(R_{+}^{\mathbb{S}_{n}}\right)$, indexed by permutations $w \in \mathbb{S}_{n}$.

Definition 2.11. For each $w \in \mathbb{S}_{n}$ we define $\mathfrak{S}_{w}^{\prime}\left(x_{1}, \ldots, x_{n}\right)$ to be the leading term of $\mathfrak{S}_{w}\left(x_{1}, \ldots, x_{n}\right)$ in the lexicographic order (2.9).

Being the leading terms of a basis of $R /\left(R_{+}^{\mathbb{S}_{n}}\right)$, it follows by the theory of Groebner bases (see 2.2 ) that also the monomials $\mathfrak{S}_{w}^{\prime}\left(x_{1}, \ldots, x_{n}\right)$ give a basis.

Remark 2.12. We already noticed in Example 2.5 that $R /\left(R_{+}^{\mathbb{S}_{n}}\right) \cong R_{\boldsymbol{b}}$ for $\boldsymbol{b}=$ $(n, n-1, \ldots, 1)$. Hence we already have a monomial basis of $R /\left(R_{+}^{\mathbb{S}_{n}}\right)$ given by Proposition 2.3. In fact, this basis coincides with the basis $\left\{\mathfrak{S}_{w}^{\prime}\left(x_{1}, \ldots, x_{n}\right) \mid w \in\right.$ $\left.\mathbb{S}_{n}\right\}$; the advantage of using Schubert polynomials is that they give us a way to index these basis elements through permutations.

There is an easy way to construct the monomials $\mathfrak{S}_{w}^{\prime}\left(x_{1}, \ldots, x_{n}\right)$ (cf. [BJS93]): let $c_{i}=\#\left\{j<w^{-1}(i) \mid w(j)>i\right\}$; then $\mathfrak{S}_{w}^{\prime}\left(x_{1}, \ldots, x_{n}\right)=x_{1}^{c_{1}} \cdots x_{n-1}^{c_{n-1}}$.

Example 2.13. The following table contains the Schubert polynomials and the polynomials $\mathfrak{S}_{w}^{\prime}$ in the case $n=3$.

\begin{tabular}{c|c|c}
$w \in \mathbb{S}_{3}$ & $\mathfrak{S}_{w}$ & $\mathfrak{S}_{w}^{\prime}$ \\
\hline$e$ & 1 & 1 \\
$s$ & $x_{1}$ & $x_{1}$ \\
$t$ & $x_{1}+x_{2}$ & $x_{2}$ \\
$s t$ & $x_{1} x_{2}$ & $x_{1} x_{2}$ \\
$t s$ & $x_{1}^{2}$ & $x_{1}^{2}$ \\
$w_{3}$ & $x_{1}^{2} x_{2}$ & $x_{1}^{2} x_{2}$
\end{tabular}




\section{Some Canonical Bases elements}

In this section we recall the definition of the bar involution and the canonical basis of the Hecke algebra for the symmetric group $\mathbb{S}_{n}$. We will then compute some canonical basis elements in the Hecke algebra for some special permutations of the symmetric group $\mathbb{S}_{n}$. We will need these expressions to compute the dimension of Soergel modules in the next section.

We remark that all actions of $\mathbb{S}_{n}$ will be from the right.

3.1. Hecke algebra. Let $s_{1}, \ldots, s_{n-1}$ denote the simple reflections which generate the symmetric group $\mathbb{S}_{n}$. For $w \in \mathbb{S}_{n}$ we let $\ell(w)$ be the length of $w$. Moreover, we denote by $\prec$ the Bruhat order on $\mathbb{S}_{n}$.

The Hecke algebra of the symmetric group $W=\mathbb{S}_{n}$ is the unital associative $\mathbb{C}(v)$-algebra $\mathcal{H}_{n}$ generated by $\left\{H_{i} \mid i=1, \ldots, n-1\right\}$ with relations

$$
\begin{aligned}
H_{i} H_{j} & =H_{j} H_{i} \quad \text { if }|i-j|>2, \\
H_{i} H_{i+1} H_{i} & =H_{i+1} H_{i} H_{i+1}, \\
H_{i}^{2} & =\left(v^{-1}-v\right) H_{i}+1 .
\end{aligned}
$$

Note that we use Soergel's normalization [Soe97] instead of Lusztig's. It follows from (3.1c) that the elements $H_{i}$ are invertible with $H_{i}^{-1}=H_{i}+v-v^{-1}$. For $w \in \mathbb{S}_{n}$ such that $w=s_{i_{1}} \cdots s_{i_{r}}$ is a reduced expression, we define $H_{w}=H_{i_{1}} \cdots H_{i_{r}}$. Thanks to (3.1b), this does not depend on the chosen reduced expression. The elements $H_{w}$ for $w \in W$ form a basis of $\mathcal{H}_{n}$, called standard basis, and we have

$$
H_{w} H_{i}= \begin{cases}H_{w s_{i}} & \text { if } \ell\left(w s_{i}\right)>\ell(w), \\ H_{w s_{i}}+\left(v^{-1}-v\right) H_{w} & \text { otherwise. }\end{cases}
$$

We can define on $\mathcal{H}_{n}$ a bar involution by $\overline{H_{w}}=H_{w^{-1}}^{-1}$ and $\bar{v}=v^{-1}$; in particular $\overline{H_{i}}=H_{i}+v-v^{-1}$. We also have a bilinear form $\langle-,-\rangle$ on $\mathcal{H}_{n}$ such that the standard basis elements are orthonormal:

$$
\left\langle H_{w}, H_{w^{\prime}}\right\rangle=\delta_{w, w^{\prime}} \quad \text { for all } w, w^{\prime} \in W .
$$

By standard arguments one can prove the following:

Proposition 3.1 ([KL79], in the normalization of [Soe97]). There exists a unique basis $\left\{C_{w} \mid w \in W\right\}$ of $\mathcal{H}_{n}$ consisting of bar-invariant elements such that

$$
C_{w}=H_{w}+\sum_{w^{\prime} \prec w} \mathcal{P}_{w^{\prime}, w}(v) H_{w^{\prime}}
$$

with $\mathcal{P}_{w^{\prime}, w} \in v \mathbb{Z}[v]$ for every $w^{\prime} \prec w$.

The basis $C_{w}$ is called the Kazhdan-Lusztig basis. We will also call it the canonical basis of $\mathcal{H}_{n}$.

Remark 3.2. There is an inductive way to construct the canonical basis elements. First, note that $C_{e}=H_{e}$. Then set $C_{i}=H_{i}+v$ : since $C_{i}$ is bar invariant, we must have $C_{s_{i}}=C_{i}$. Now suppose $w=w^{\prime} s_{i} \succ w^{\prime}$; then $C_{w^{\prime}} C_{i}$ is bar invariant and is equal to $H_{w}$ plus a $\mathbb{Z}\left[v, v^{-1}\right]$-linear combination of some $H_{w^{\prime \prime}}$ for $w^{\prime \prime} \prec w$. It follows that

$$
C_{w^{\prime}} C_{i}=C_{w}+p \quad \text { for some } p \in \bigoplus_{w^{\prime \prime} \prec w} \mathbb{Z} C_{w^{\prime \prime}}
$$


3.2. Combinatorics. Let us fix an integer $0 \leq k \leq n$. If $s_{1}, \ldots, s_{n-1}$ are the simple reflections in $\mathbb{S}_{n}$, let $W_{k}$ be the subgroup generated by $s_{1}, \ldots, s_{k-1}$ and $W_{k}^{\perp}$ be the subgroup generated by $s_{k+1}, \ldots, s_{n-1}$. Notice that $\mathbb{S}_{k} \times \mathbb{S}_{n-k} \cong W_{k} \times W_{k}^{\perp} \subseteq$ $\mathbb{S}_{n}$. Let $w_{k}$ be the longest element of $W_{k}$, and let $D=D_{n, k}$ be the set of shortest coset representatives $\left(W_{k} \times W_{k}^{\perp} \backslash \mathbb{S}_{n}\right)^{\text {short }}$. The set $D$ is in natural bijection with $\wedge \vee$-sequences consisting of $k \wedge$ 's and $n-k \vee$ 's, by mapping the identity $e \in \mathbb{S}_{n}$ to the sequence

$$
e=\underbrace{\wedge \cdots \wedge}_{k} \underbrace{\vee \cdots \vee}_{n-k}
$$

and letting $\mathbb{S}_{n}$ act by permutation of positions; in order to obtain a bijection with right coset representatives we regard this as a right action. From now on, we identify an element $z \in D$ with the corresponding $\wedge \vee$-sequence.

There are a few ways to encode an element $z \in D$, which we are now going to explain.

The position sequences: In an $\wedge \vee$-sequence $z \in D$, we number the $\wedge$ 's (resp. the $\checkmark$ 's) from 1 to $k$ (resp. from 1 to $n-k$ ) from the left to the right. Moreover, we number the positions of a $\wedge \vee$-sequence from 1 to $n$ from the left to the right. We let $\wedge_{i}^{z}$ be the position of the $i$-th $\wedge$ and $\vee_{j}^{z}$ be the position of the $j$-th $\vee$ in $z$. For example, in the sequence

$$
z=\wedge \vee \vee \wedge \vee \wedge \wedge
$$

we have $\wedge_{2}^{z}=4$ and $\vee_{1}^{z}=2$. Notice that both the sequences $\left(\wedge_{1}^{z}, \ldots, \wedge_{k}^{z}\right)$ and $\left(\vee_{1}^{z}, \ldots, \vee_{n-k}^{z}\right)$ uniquely determine $z$.

The $\wedge$-distance sequence: We set

$$
z_{i}^{\wedge}=\wedge_{i}^{z}-i \quad \text { for } i=1, \ldots, k,
$$

so that

$$
\left(\wedge_{1}^{z}, \ldots, \wedge_{k}^{z}\right)=\left(1+z_{1}^{\wedge}, \ldots, k+z_{k}^{\wedge}\right) .
$$

In other words, $z_{i}^{\wedge}$ measures how many steps the $i$-th $\wedge$ of the initial sequence $e$ has been moved to the right by the permutation $z$. This defines a bijection $z \mapsto \boldsymbol{z}^{\wedge}$ between $D$ and the set

$$
\left\{\boldsymbol{z}^{\wedge}=\left(z_{1}^{\wedge}, \ldots, z_{k}^{\wedge}\right) \mid 0 \leq z_{1}^{\wedge} \leq \cdots \leq z_{k}^{\wedge} \leq n-k\right\} .
$$

Define the permutation

$$
t_{i, \ell}^{\wedge}=s_{i} s_{i+1} \cdots s_{i+\ell-1}
$$

for all $i=1, \ldots, n-1$ and $\ell=1, \ldots, n-i$ (and set $t_{i, 0}^{\wedge}=e$ ). Then we have a reduced expression for $z$ :

$$
z=t_{k, z_{k}}^{\wedge} \cdots t_{1, z_{1}^{\wedge}}^{\wedge}
$$

The $\vee$-distance sequence: Analogously, set

$$
z_{i}^{\vee}=i-\vee_{k-i}^{z} \quad \text { for } i=k+1, \ldots, n
$$

so that

$$
\left(\vee_{1}^{z}, \ldots, \vee_{n-k}^{z}\right)=\left(k+1-z_{k+1}^{\vee}, \ldots, n-z_{n-k}^{\vee}\right)
$$


In other words, $z_{i}^{\vee}$ measures how many steps the $(i-k)$-th $\vee$ of $e$ has been moved to the left by the permutation $z$. This defines a bijection $z \mapsto \boldsymbol{z}^{\vee}$ between $D$ and the set

$$
\left\{\boldsymbol{z}^{\vee}=\left(z_{k+1}^{\vee}, \ldots, z_{n}^{\vee}\right) \mid k \geq z_{k+1}^{\vee} \geq \cdots \geq z_{n}^{\vee} \geq 0\right\}
$$

Define

$$
t_{k+i, \ell}^{\vee}=s_{k+i-1} s_{k+i-2} \cdots s_{k+i-\ell}
$$

for $i=1, \ldots, n-k$ and $\ell=1, \ldots, k$ (and set $t_{k+i, 0}^{\vee}=e$ ). Then we have another reduced expression for $z$ :

$$
z=t_{k+1, z_{k+1}^{\vee}}^{\vee} \cdots t_{n, z_{n}^{\vee}}^{\vee}
$$

The $\boldsymbol{b}$-sequence: Finally we want to assign to the element $z \in D$ its $\boldsymbol{b}$-sequence $\boldsymbol{b}^{z}$. Let

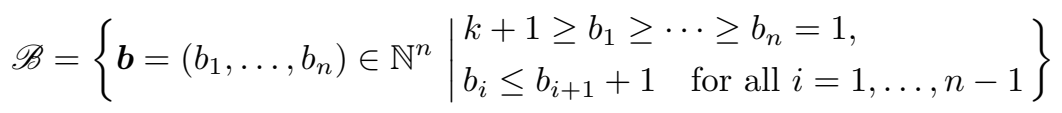

and define $\boldsymbol{b}^{z} \in \mathscr{B}$ by

$$
b_{i}^{z}=\#\left\{j \mid \wedge_{j}^{z}>i\right\}+1 .
$$

In other words, $b_{i}^{z}-1$ is the number of $\wedge$ 's on the right of position $i$. It is clear that $\boldsymbol{b}^{z}$ uniquely determines the element $z \in D$. In fact, this defines a bijection between $D$ and $\mathscr{B}$.

Example 3.3. Let $n=8, k=4$ and consider the element $z=s_{4} s_{5} s_{6} s_{3} \in D$. The corresponding $\wedge \vee$-sequence and the $\boldsymbol{b}$-sequences are:

$$
\mathbf{b}^{z}=4 \wedge \begin{aligned}
& \wedge 3 \\
& \mathbf{b}^{2}
\end{aligned}
$$

We also have $\boldsymbol{z}^{\wedge}=(0,0,1,3)$ and $\boldsymbol{z}^{\vee}=(2,1,1,0)$.

3.3. Canonical basis elements. One of the rare examples of explicitly known canonical basis elements is the following (cf. [Soe97, Prop. 2.9]):

Lemma 3.4. Let $w_{k}$ be the longest element of $\mathbb{S}_{k}$. Then the canonical basis element $C_{w_{k}}$ is given by

$$
C_{w_{k}}=\sum_{w^{\prime} \in \mathbb{S}_{k}} v^{\ell\left(w_{k}\right)-\ell\left(w^{\prime}\right)} H_{w^{\prime}} .
$$

This expression holds both in $\mathcal{H}_{k}$ and in $\mathcal{H}_{n} \supset \mathcal{H}_{k}$ for any $n>k$.

In fact, formula (3.19) generalizes verbatim to the case of the longest element of any parabolic subgroup of $\mathbb{S}_{n}$.

In the next proposition we will generalize (3.19) and give explicit formulas for the canonical basis elements $C_{w_{k} z}$ for $z \in D$. But first we introduce the following notation: we set

$$
\sum_{w^{\prime} \in \mathbb{S}_{k}}^{(v)} f\left(w^{\prime}\right)=\sum_{w^{\prime} \in S_{k}} v^{-\ell\left(w^{\prime}\right)} f\left(w^{\prime}\right) \quad \text { and } \quad \sum_{i=0}^{h} g(i)=\sum_{i=0}^{h} v^{-i} g(i)
$$

for whatever functions $f$ defined on $\mathbb{S}_{n}$ and $g$ defined on $\{0, \ldots, h\}$. 
Proposition 3.5. Let $z \in D$, with $z=t_{k+1, z_{k+1}^{\vee}}^{\vee} \cdots t_{n, z_{n}^{\vee}}^{\vee}$. Then

$$
C_{w_{k} z}=\sum_{w^{\prime} \in \mathbb{S}_{k}}^{(v)} \sum_{i_{k+1}=0}^{z_{k+1}^{\vee}} \cdots \sum_{i_{n}=0}^{z_{n}^{\vee}}(v) v^{\ell\left(w_{k} z\right)} H_{w^{\prime} t_{k+1, i_{k+1}}^{\vee} \cdots t_{n, i_{n}}^{\vee}} .
$$

Proof. First, we note that all words $w^{\prime} t_{k+1, i_{k+1}}^{\vee} \cdots t_{n, i_{n}}^{\vee}$ that appear in the expression on the right are actually reduced words. This is clear if we look at the action of this permutation on the string

$$
\wedge_{1} \cdots \wedge_{k} \vee_{k+1} \cdots \vee_{n}
$$

from the right: the length of the permutation is the cardinality of the set $X$ of the couples of symbols of this string that have been inverted. To $X$ belong $\ell\left(w^{\prime}\right)$ couples consisting of two $\wedge$ 's; moreover, every $\vee_{k+\alpha}$ appears in $X$ exactly $i_{\alpha}$ times coupled with some $\wedge$ or some $\vee_{k+\beta}$ for $\beta<\alpha$. Hence the length of the permutation $w^{\prime} t_{k+1, i_{k+1}}^{\vee} \cdots t_{n, i_{n}}^{\vee}$ is exactly $\ell\left(w^{\prime}\right)+i_{k+1}+\cdots+i_{n}$, and therefore this is a reduced expression.

Now, in the r.h.s. of (3.21) the coefficient of $H_{w_{k} z}$ is one, while the coefficient of every other basis element $H_{w^{\prime}} t_{k+1, i_{k+1}}^{\vee} \cdots t_{n, i_{n}}^{\vee}$ is divisible by $v$. Hence the only thing we have to prove is that the r.h.s of (3.21) is bar invariant.

We proceed by induction on the length of $z$, the case $z=0$ being given by Lemma 3.4. Let $h$ be the greatest index such that $z_{h}^{\vee} \neq 0$. Hence we have $z=$ $t_{k+1, z_{k+1}^{\vee}}^{\vee} \cdots t_{h, z_{h}^{\vee}}^{\vee}$. First suppose that $z_{h}^{\vee} \geq 2$. Set $z^{\prime}=t_{k+1, z_{k+1}^{\vee}}^{\vee} \cdots t_{h, z_{h}^{\vee}-1}^{\vee}$ and $j=h-z_{h}^{\vee}$ so that $z=z^{\prime} s_{j}$. We compute:

$$
\begin{aligned}
& C_{w_{k} z^{\prime}} C_{j}=\left(\sum_{w^{\prime} \in \mathbb{S}_{k}}^{(v)} \sum_{i_{k+1}=0}^{z_{k+1}^{\vee}} \cdots \sum_{i_{h}=0}^{z_{h}^{\vee}-1} v^{\ell\left(w_{k} z^{\prime}\right)} H_{w^{\prime} t_{k+1, i_{k+1}}^{\vee} \cdots t_{h, i_{h}}^{\vee}}\right)\left(H_{j}+v\right) \\
& =\sum_{w^{\prime} \in \mathbb{S}_{k}}^{(v)} \sum_{i_{k+1}=0}^{z_{k+1}^{\vee}} \cdots \sum_{i_{h-1}=0}^{z_{h-1}^{\vee}} v^{\ell\left(w_{k} z^{\prime}\right)-z_{h}^{\vee}+1} H_{w^{\prime} t_{k+1, i_{k+1}}^{\vee} \cdots t_{h-1, i_{h-1}}^{\vee} t_{h, z_{h}^{\vee}}^{\vee}} \\
& +\left(\sum_{w^{\prime} \in \mathbb{S}_{k}}^{(v)} \sum_{i_{k+1}=0}^{z_{k+1}^{\vee}} \cdots \sum_{i_{h}=0}^{(v)} z_{h}^{\vee}-2\right. \\
& \quad+\sum_{w^{\prime} \in \mathbb{S}_{k}}^{(v)} \sum_{i_{k+1}=0}^{\ell\left(w_{k} z^{\prime}\right)} H_{w^{\prime} t_{k+1, i_{k+1}}^{\vee} \cdots t_{h, i_{h}}^{\vee}}^{(v)} \cdots \sum_{i_{h}=0}^{\vee}{ }^{\vee}-1
\end{aligned}
$$

The element $C_{w_{k} z^{\prime}} C_{j}$ is obviously bar-invariant. Moreover, the sum of (3.24) and (3.26) gives exactly the r.h.s. of (3.21) for $C_{w_{k} z}$; hence we only need to prove that (3.25) is bar invariant. But it is easy to check that in (3.25) the term $H_{j}$ on the right acts as $v^{-1}$; hence (3.25) is equal to the r.h.s. of (3.21) for $C_{w_{k} z^{\prime \prime}}$, where $z^{\prime \prime}=t_{k+1, z_{k+1}^{\vee}}^{\vee} \cdots t_{h, z_{h}^{\vee}-2}^{\vee}$, and this is bar-invariant by induction.

Now suppose instead that $z_{h}^{\vee}=1$, and set $z^{\prime}=t_{k+1, z_{k+1}^{\vee}}^{\vee} \cdots t_{h-1, z_{h-1}^{\vee}}^{\vee}$ so that $z=z^{\prime} s_{h-1}$. Then a computation shows that $C_{w_{k} z^{\prime}} C_{h-1}$ is equal to the r.h.s. of (3.21) for $C_{w_{k} z}$, which is therefore bar-invariant (see [Sar14 for more details). 
Let $z \in D$, with $z=t_{k+1, z_{k+1}^{\vee}}^{\vee} \cdots t_{n, z_{n}^{\vee}}^{\vee}$, and suppose that for some index $j$ we have $z_{j}^{\vee}=z_{j+1}^{\vee}$. Then in a similar way as above it is possible to compute explicitly the canonical basis element $C_{s_{j} w_{k} z}$. We refer to Sar14 for a detailed computation and we state instead the following corollary, which is all we will use later:

Corollary 3.6. Let $z \in D$, with $z=t_{k+1, z_{k+1}^{\vee}}^{\vee} \cdot t_{n, z_{n}^{\vee}}^{\vee}$. Suppose that for some index $j$ we have $z_{j}^{\vee}=z_{j+1}^{\vee}$. Then the canonical basis element $C_{s_{j} w_{k} z}$ is a sum of

$$
k !\left(z_{k+1}^{\vee}+1\right) \cdots\left(z_{j}^{\vee}+1\right)\left(z_{j+1}^{\vee}+2\right)\left(z_{j+2}^{\vee}+1\right) \cdots\left(z_{n}^{\vee}+1\right)
$$

standard basis elements with monomial coefficients in $v$.

\section{Soergel modules}

In this section we will describe some Soergel modules as quotient rings $R_{\boldsymbol{b}}$ (defined in Section 2). The strategy is the following: we prove that the ideal $I_{\boldsymbol{b}}$ is contained in the annihilator and we then use a dimension argument comparing the dimension of $R_{\boldsymbol{b}}$ (Proposition 2.3) and of the Soergel module (given by the corresponding canonical basis element computed in Section 3). We compute then the homomorphism spaces between these Soergel modules (\$4.3).

Fix a positive integer $n$ and let $R=\mathbb{C}\left[x_{1}, \ldots, x_{n}\right]$ be the polynomial ring in $n$ variables. For $0 \leq \ell \leq n$ let $J_{\ell}$ be the ideal generated by the non-constant symmetric polynomials in $\ell$ variables $x_{1}, \ldots, x_{\ell}$. Let moreover $B=\mathbb{C}\left[x_{1}, \ldots, x_{n}\right] / J_{n}$. For a simple reflection $s_{i} \in \mathbb{S}_{n}$, let $B^{s_{i}}$ denote the invariants under $s_{i}$. In the following, we will abbreviate $\otimes_{B^{s_{i}}}$ by $\otimes_{i}$ while $\otimes$ will be simply $\otimes_{B}$. We let also $B_{i}=B \otimes_{i} B$.

4.1. Soergel modules. We now define Soergel modules for the symmetric group $\mathbb{S}_{n}$ by recursion on the Bruhat ordering. First we set $C_{e}=\mathbb{C}$. Then let $w \in \mathbb{S}_{n}$ be a permutation and choose some reduced expression $w=s_{i_{1}} \cdots s_{i_{r}}$ where $s_{i_{1}}, \ldots, s_{i_{r}} \in$ $\mathbb{S}_{n}$ are simple reflections. Then we have:

Theorem 4.1 ( Soe90]). The $B$-module $B_{i_{r}} \otimes \cdots \otimes B_{i_{1}} \otimes \mathbb{C}$ has a unique indecomposable direct summand $\mathrm{C}_{w}$ which is not isomorphic to some $\mathrm{C}_{w^{\prime}}$ for $w^{\prime} \prec w$. This is the unique indecomposable summand containing $1 \otimes \cdots \otimes 1$. Up to isomorphism, $\mathrm{C}_{w}$ does not depend on the particular reduced expression chosen for $w$.

We call the $\mathbf{C}_{w}$ 's for $w \in \mathbb{S}_{n}$ Soergel modules.

Example 4.2. Consider a simple reflection $s_{i} \in \mathbb{S}_{n}$. According to the theorem, the indecomposable object $\mathrm{C}_{i}=\mathrm{C}_{s_{i}}$ is a summand of $B_{i} \otimes \mathbb{C}$. But it is immediate to check that the two dimensional $B$-module $B_{i} \otimes \mathbb{C}$ is indecomposable, hence $\mathrm{C}_{i}=$ $B_{i} \otimes \mathbb{C}$. This module is in fact isomorphic to $R /\left(x_{1}, \ldots, x_{i-1}, x_{i}+x_{i+1}, x_{i+2}, \ldots, x_{n}\right)$.

Notice that since $B$ is a quotient of $R$ we have $\operatorname{Hom}_{B}(M, N) \cong \operatorname{Hom}_{R}(M, N)$ for all $M, N \in B$-mod. In other words, the category of $B$-modules embeds as a full subcategory into the category of $R$-modules. Hence, it is harmful to consider $B$-modules as $R$-modules.

To compute Soergel modules we will need to know their dimension. This is given from the Kazhdan-Lusztig conjecture:

Theorem 4.3. For all $w \in \mathbb{S}_{n}$ we have

$$
\operatorname{dim}_{\mathbb{C}} \mathrm{C}_{w}=\sum_{z \preceq w} \mathcal{P}_{z, w}(1),
$$

where the $\mathcal{P}_{z, w}$ 's are the Kazhdan-Lusztig polynomials (3.4). 
Proof. For the proof, we need some facts about the BGG category $\mathcal{O}$ (see Hum08] and Section 6 below); we use the notation from $\$ 6.2$. By Theorem 6.3 we have $\mathrm{C}_{w} \cong \operatorname{Hom}_{B}\left(B, \mathrm{C}_{w}\right) \cong \operatorname{Hom}_{\mathcal{O}}\left(P\left(w_{0} \cdot 0\right), P(w \cdot 0)\right)$. Hence

$$
\operatorname{dim}_{\mathbb{C}} \mathrm{C}_{w}=\operatorname{dim}_{\mathbb{C}} \operatorname{Hom}_{\mathcal{O}}\left(P\left(w_{0} \cdot 0\right), P(w \cdot 0)\right)=\left[P(w \cdot 0): L\left(w_{0} \cdot 0\right)\right],
$$

where the latter denotes the multiplicity of the simple module $L\left(w_{0} \cdot 0\right)$ in some composition series of $P(w \cdot 0)$. Since $P(w \cdot 0)$ has a Verma filtration, and since $\left[M(z \cdot 0): L\left(w_{0} \cdot 0\right)\right]=1$ for all Verma modules $M(z \cdot 0)$, we have further that $\left[P(w \cdot 0): L\left(w_{0} \cdot 0\right)\right]=\sum_{z \in \mathbb{S}_{n}}(P(w \cdot 0): M(z \cdot 0))$, where $(P(w \cdot 0): M(z \cdot 0))$ denotes the multiplicity of $M(z \cdot 0)$ in some Verma filtration of $P(w \cdot 0)$. The Kazhdan-Lusztig conjecture KL79] (see [EW12 for a proof) states precisely that $(P(w \cdot 0): M(z \cdot 0))=\mathcal{P}_{z, w}(1)$, and this concludes the proof (notice that $\mathcal{P}_{z, w}(1)=0$ unless $z \preceq w)$.

4.2. Some Soergel modules. We determine now explicitly some modules $C_{w}$. In the following, we will use the notation introduced in $\$ 3.2$. We recall the following well-known fact:

Lemma 4.4. As a $\mathbb{C}$-vector space, a basis of $B \otimes_{i_{1}} B \otimes \cdots \otimes_{i_{r-1}} B \otimes_{i_{r}} \mathbb{C}$ is given by

$$
\left\{x_{i_{1}}^{\varepsilon_{1}} \otimes \cdots \otimes x_{i_{r}}^{\varepsilon_{r}} \otimes 1 \mid \varepsilon_{j} \in\{0,1\}\right\} .
$$

Proof. The claim follows since each polynomial $f \in R$ can be written uniquely as $f=P_{i}(f)+x_{i} \partial_{i}(f)$, with $P_{i}(f), \partial_{i}(f) \in R^{s_{i}}$; cf. (2.5).

A key tool to determine the Soergel modules $\mathrm{C}_{w_{k} z}$ is given by the next proposition; unfortunately it is based on a lemma which uses facts about the BGG category $\mathcal{O}$, and that we hence postpone to Section 6 .

Proposition 4.5. For all $z \in D$ the module $\mathrm{C}_{w_{k} z}$ is cyclic. In particular, $\mathrm{C}_{w_{k} z} \cong$ $R / \operatorname{Ann}_{R} \mathrm{C}_{w_{k} z} \cong B / \operatorname{Ann}_{B} \mathrm{C}_{w_{k} z}$.

Proof. By Proposition 3.5, $H_{e}$ appears exactly once with coefficient $v^{\ell\left(w_{k} z\right)}$ in the canonical basis element $C_{w_{k}} z$. By Lemma 6.4 this implies that $\mathrm{C}_{w_{k} z}$ is cyclic.

Lemma 4.6. For every $z \in D$ the dimension of $\mathrm{C}_{w_{k} z}$ over $\mathbb{C}$ is given by

$$
\operatorname{dim}_{\mathbb{C}} C_{w_{k} z}=k !\left(z_{k+1}^{\vee}+1\right) \cdots\left(z_{n}^{\vee}+1\right)=b_{1}^{z} \cdots b_{n}^{z} .
$$

Proof. The first equality follows directly from Theorem 4.3 and Proposition 3.5 . We want to show the second equality. As in Example 3.3. we imagine the $\boldsymbol{b}$-sequence written on top of the $\wedge \vee$-sequence for $z$. Over the $\wedge$ 's we have the numbers between 1 and $k$, each appearing once: hence their contribute is $k$ !. Over the $j$-th $\vee$, we have a number measuring how many $\wedge$ 's are on its right, plus one: this coincides with how many times this $\vee$ has been moved to the left plus one, that is, $z_{k+j}^{\vee}+1$. The claim follows immediately.

Lemma 4.7. The module $\mathrm{C}_{w_{k}}$ is isomorphic to $R /\left(J_{k}, x_{k+1}, \ldots, x_{n}\right)$.

Proof. Let $J^{\prime}=\left(J_{k}, x_{k+1}, \ldots, x_{n}\right)$. By Proposition 4.5, the module $\mathrm{C}_{w_{k}}$ is cyclic over $B$. Choose any reduced expression $s_{i_{1}} \cdots s_{i_{N}}$ for $w_{k}$ and build the corresponding module $B_{w_{k}}=B_{i_{N}} \otimes \cdots \otimes B_{i_{1}} \otimes \mathbb{C}$. Since all polynomials of $J^{\prime}$ are symmetric in the first $k$ variables, we have $J^{\prime} \subseteq \operatorname{Ann}_{R} B_{w_{k}} \subseteq \operatorname{Ann}_{R} C_{w_{k}}$; hence $C_{w_{k}}$ is a quotient of $R / J^{\prime}$. Notice that $J^{\prime}=I_{\boldsymbol{b}^{e}}$ for $e \in \mathbb{S}_{n}$ the identity element. By Lemma 4.6 and Proposition 2.3, $\operatorname{dim}_{\mathbb{C}} C_{w_{k}}=\operatorname{dim}_{\mathbb{C}} R / J^{\prime}$, hence $C_{w_{k}}=R / J^{\prime}$. 
As we said, we will use the same notation of $\$ 3.2$. For $t_{i, \ell}^{\wedge}($ see (3.10) $)$, let

$$
B_{t_{i, \ell}}=B_{i+\ell-1} \otimes B_{i+\ell-2} \otimes \cdots \otimes B_{i}
$$

and for $z=t_{k, z_{k}^{\wedge}}^{\wedge} \cdots t_{1, z_{1}^{\wedge}}^{\wedge}$ let

$$
B_{z}^{\wedge}=B_{t_{1, z_{1}}^{\wedge}} \otimes \cdots \otimes B_{t_{k, z_{\hat{k}}}} .
$$

Moreover, for $t_{i, \ell}^{\vee}($ see (3.15) $)$, let

$$
B_{t_{i, \ell}^{\vee}}=B_{i-\ell} \otimes B_{i-\ell+1} \otimes \cdots \otimes B_{i-1},
$$

and for $z=t_{k+1, z_{k+1}^{\vee}}^{\vee} \cdots t_{n, z_{n}^{\vee}}^{\vee}$ let

$$
B_{z}^{\wedge}=B_{t_{n, z_{n}^{\vee}}^{\vee}} \otimes \cdots \otimes B_{t_{k+1, z_{k+1}^{\vee}}^{\vee}} .
$$

From Soergel Theorem 4.1 and Proposition 4.5, it follows that $C_{w_{k} z}$ is isomorphic both to the $B$-submodule of $B_{z}^{\wedge} \otimes \mathrm{C}_{w_{k}}$ generated by $\underline{1}=1 \otimes \cdots \otimes 1$ and to the $B$-submodule of $B_{z}^{\vee} \otimes \mathrm{C}_{w_{k}}$ generated by $\underline{1}=1 \otimes \cdots \otimes 1$.

The following lemma is the crucial step to determine the annihilator of $C_{w_{k} z}$.

Lemma 4.8. Let $z \in D$, and let $m$ be the number of non-zero $z_{i}^{\wedge}$ 's. Then

$$
h_{\ell}\left(x_{1}, \ldots, x_{k-m+z_{k-m+1}^{\wedge}}\right) \in \operatorname{Ann~}_{w_{k} z}
$$

for all $\ell>m$.

Proof. Let us prove the assertion by induction on the sum $N$ of the $z_{i}^{\wedge}$ 's (that is, up to a shift, the length of $z$ ). If $N=0$, then also $m=0$, and $h_{\ell}\left(x_{1}, \ldots, x_{k}\right) \in$ Ann $C_{w_{k}}=\left(J_{k}, x_{k+1}, \ldots, x_{n}\right)$ for every $\ell>1$ by Lemma 4.7 .

For the inductive step, let $i=k-m+z_{k-m+1}^{\wedge}$, write $z=z^{\prime} s_{i}$ and compute in $B \otimes_{i}\left(B_{z^{\prime}}^{\wedge} \otimes \mathrm{C}_{w_{k}}\right):$

$$
\begin{aligned}
h_{\ell+1} & \left(x_{1}, \ldots, x_{i}\right) \cdot(1 \otimes 1) \\
\quad & =\left(P_{i}\left(h_{\ell+1}\left(x_{1}, \ldots, x_{i}\right)\right)+x_{i} \partial_{i}\left(h_{\ell+1}\left(x_{1}, \ldots, x_{i}\right)\right)\right) \cdot 1 \otimes 1 \\
& =1 \otimes\left(P_{i}\left(h_{\ell+1}\left(x_{1}, \ldots, x_{i}\right)\right) \cdot 1\right)+x_{i} \otimes\left(\partial_{i}\left(h_{\ell+1}\left(x_{1}, \ldots, x_{i}\right)\right) \cdot 1\right) .
\end{aligned}
$$

Since $C_{w_{k} z}$ is a summand of $B \otimes_{i}\left(B_{z^{\prime}}^{\vee} \otimes C_{w_{k}}\right)$, it is sufficient to show that (4.10) is zero. In fact, we prove that both terms $P_{i}\left(h_{\ell+1}\left(x_{1}, \ldots, x_{i}\right)\right)$ and $\partial_{i}\left(h_{\ell+1}\left(x_{1}, \ldots, x_{i}\right)\right)$ act as 0 on $B_{z^{\prime}}^{\wedge}$.

Let us start considering the second term. Let $y \in D$ be determined by $y_{i}^{\wedge}=z_{i}^{\wedge}$ for $i \neq k-m+1, k-m+2$, while $y_{k-m+1}^{\wedge}=0$ and $y_{k-m+2}^{\wedge}=z_{k-m+1}^{\wedge}$. Notice that our chosen reduced expression for $z$ splits as $z^{\prime}=y z^{\prime \prime}$, so that

$$
B_{z^{\prime}}^{\wedge}=B_{i-1} B_{i-2} \cdots B_{k-m+1} B_{j} B_{j-1} \cdots B_{i+2} B_{y}^{\wedge}=B_{z^{\prime \prime}} B_{y}^{\wedge}
$$

for $j=k-m+1+z_{k-m+2}^{\wedge}$, where we omitted the tensor product signs. By (2.6), $\partial_{i}\left(h_{\ell+1}\left(x_{1}, \ldots, x_{i}\right)\right)=h_{\ell}\left(x_{1}, \ldots, x_{i+1}\right)$; being symmetric in the variables $x_{a}$ for $a \neq i, i+1$, this passes through $B_{z^{\prime \prime}}$ and acts on $B_{y}^{\wedge} \otimes \mathrm{C}_{w_{k}}$. By induction, this action is zero.

Now let us consider the action of the term $P_{i}\left(h_{\ell+1}\left(x_{1}, \ldots, x_{i}\right)\right)$. Write

$$
\begin{aligned}
P_{i}\left(h_{\ell+1}\left(x_{1}, \ldots, x_{i}\right)\right) & =h_{\ell+1}\left(x_{1}, \ldots, x_{i}\right)-x_{i} \partial_{i} h_{\ell+1}\left(x_{1}, \ldots, x_{i}\right) \\
& =h_{\ell+1}\left(x_{1}, \ldots, x_{i}\right)-x_{i} h_{\ell}\left(x_{1}, \ldots, x_{i+1}\right) .
\end{aligned}
$$


Of these two summands, the second acts as zero exactly as before. For the first one, write $y^{\prime} s_{i+1}=y$ so that $B_{y}^{\wedge}=B \otimes_{i+1} B_{y^{\prime}}^{\wedge}$. Then $h_{\ell+1}\left(x_{1}, \ldots, x_{i}\right)$ commutes with the first tensor product and by induction acts as zero on $B_{y^{\prime}}^{\wedge}$.

Proposition 4.9. Let $z \in D$ with corresponding $\boldsymbol{b}$-sequence $\boldsymbol{b}^{z}$. Then the complete symmetric polynomial $h_{b_{i}^{z}}\left(x_{1}, \ldots, x_{i}\right)$ lies in $\mathrm{Ann}_{w_{k} z}$ for all $i=1, \ldots, n$.

Proof. We divide the indices $i \in\{1, \ldots, n\}$ corresponding to the positions in the $\wedge \vee$-sequence of $z$ in three subsets: we call an index $i$ such that $z_{i}^{\wedge}=0$ initial, we call an index $i$ for which $b_{i}^{z}=1$ final, and we call all other indices in between in the middle:

$$
\underbrace{\wedge \cdots \wedge}_{\text {initial }} \underbrace{\vee \times \cdots \times}_{\text {middle }} \underbrace{\wedge \vee \cdots \vee}_{\text {final }}
$$

where $\mathrm{a} \times$ stands for a $\wedge$ or a $\vee$. Notice that it can happen that an index $i$ is both initial and final if and only if there are no $\vee$ 's, that is, $k=n$. Since in this case we already know the claim, we can exclude it.

If $i$ is final, then $w_{k} z \in \mathbb{S}_{i} \subset \mathbb{S}_{n}$ (where $\mathbb{S}_{i}$ is the subgroup generated by the first $i-1$ simple transpositions) and obviously $h_{1}\left(x_{1}, \ldots, x_{i}\right)$ annihilates $\mathrm{C}_{w_{k} z}$.

If $z$ is not the identity (in which case there are no indexes in the middle), then $i=k-h+z_{k-h+1}^{\wedge}$ is in the middle, and the statement of Lemma 4.8 is that $h_{b_{i}^{z}}\left(x_{1}, \ldots, x_{i}\right) \in \operatorname{Ann} C_{w_{k} z}$. For the other indexes in the middle, we can use Lemma 4.8 after letting $h_{b_{i}^{z}}\left(x_{1}, \ldots, x_{i}\right)$ commute with some initial tensor symbols of $B_{z}^{\wedge}$.

If $i$ is initial, then $z$ is a permutation in the subgroup of $\mathbb{S}_{n}$ generated by $s_{i+1}, \ldots, s_{n-1}$; hence $h_{b_{i}^{z}}\left(x_{1}, \ldots, x_{i}\right)$, when acting on $B_{z}^{\wedge} \otimes \mathrm{C}_{w_{k}}$, can pass through $B_{z}^{\wedge}$, and we only need to prove that $h_{b_{i}^{z}}\left(x_{1}, \ldots, x_{i}\right) \in \mathrm{Ann}_{w_{k}}$. In fact, renaming the indexes this follows from the following general statement: $h_{a}\left(x_{1}, \ldots, x_{\ell}\right) \in J_{k}$ for every $1 \leq \ell \leq k$ and $a>k-\ell$. This well-known fact can easily be proved by (reversed) induction on $h$ : if $h=k$ the claim is obvious; for the inductive step, just use (2.3).

We now identify the Soergel modules with the rings $R_{b}=R / I_{b}$ defined in $\$ 2.2$.

Theorem 4.10. Let $z \in D$ with corresponding $\boldsymbol{b}$-sequence $\boldsymbol{b}^{z}$. Then $\mathrm{Ann}_{\mathrm{w}_{k} z}=$ $I_{\boldsymbol{b}^{z}}$ and $\mathrm{C}_{w_{k} z} \cong R_{\boldsymbol{b}^{z}}$. A basis of $R_{\boldsymbol{b}^{z}}$ is given by

$$
\left\{x_{1}^{c_{1}} \cdots x_{n-1}^{c_{n-1}} \cdot \underline{1} \mid 0 \leq c_{i}<b_{i}^{z}\right\} .
$$

Proof. Let $\boldsymbol{b}=\boldsymbol{b}^{z}$. By Proposition 4.9, $I_{\boldsymbol{b}} \subseteq$ Ann $C_{w_{k} z}$, so we have a surjective map $R / I_{\boldsymbol{b}} \rightarrow R /\left(\operatorname{Ann} \mathrm{C}_{w_{k} z}\right)$. By Proposition 2.3 and Lemma 4.6 their dimensions agree, hence $I_{\boldsymbol{b}}=\mathrm{Ann}_{\mathrm{w}_{k} z}$. The basis of $R_{\boldsymbol{b}}$ is given by Proposition 2.3

Translating Proposition 2.6. we can determine the homomorphism spaces between the Soergel modules $\mathrm{C}_{w_{k} z}$ :

Corollary 4.11. Let $z, z^{\prime} \in D$ with $\boldsymbol{b}$-sequences $\boldsymbol{b}^{z}, \boldsymbol{b}^{z^{\prime}}$. Let $c_{i}=\max \left\{b_{i}^{z^{\prime}}-b_{i}^{z}, 0\right\}$ for $i=1, \ldots, n-1$. Then a $\mathbb{C}$-basis of $\operatorname{Hom}_{R}\left(\mathrm{C}_{w_{k} z}, \mathrm{C}_{w_{k} z^{\prime}}\right)$ is given by

$$
\left\{1 \mapsto x_{1}^{j_{1}} \cdots x_{n-1}^{j_{n-1}} \mid c_{i} \leq j_{i}<b_{i}^{z^{\prime}}\right\} .
$$

We will need in the following some other Soergel modules corresponding to elements $w^{\prime} \in \mathbb{S}_{n}$ which differ from some $w_{k} z$ only by a simple reflection, as in the following proposition. 
Proposition 4.12. Let $z \in D$ with corresponding $\boldsymbol{b}$-sequence $\boldsymbol{b}^{z}$. Suppose $z_{j}^{\vee}=$ $z_{j+1}^{\vee}$ for some index $j$. Let $\ell=j-z_{j}^{\vee}$, so that $s_{j} z=z s_{\ell}$. Then $\mathrm{C}_{s_{j} w_{k} z}$ is the quotient of $R$ modulo the ideal generated by the complete symmetric polynomials

$$
h_{a_{i}}\left(x_{1}, \ldots, x_{i}\right) \quad \text { for } i=1, \ldots, n,
$$

where $a_{i}=\boldsymbol{b}_{i}^{z}$ for $i \neq \ell$ while $a_{\ell}=\boldsymbol{b}_{\ell+1}^{z}$.

Notice that the sequence $\boldsymbol{a}=\left(a_{1}, \ldots, a_{n}\right)$ is not an element of $\mathscr{B}^{\prime}$, since $a_{\ell}=$ $a_{\ell-1}+1$.

Proof. The proof is analogous to the proof of Theorem 4.10. By Corollary 3.6, the module $\mathrm{C}_{s_{j} w_{k} z}$ is cyclic. In particular, it is the submodule generated by 1 inside $B \otimes_{\ell} C_{w_{k} z}$. First, let us prove that the polynomials 4.15) lie in $A n n C_{s_{j} w_{k} z}$ or equivalently that they vanish on $B \otimes_{\ell} C_{w_{k} z}$. This is clear for $i \neq \ell$ : in this case, these polynomials commute with the first tensor product, and then they vanish because they lie in $\mathrm{Ann}_{w_{k} z}$ by Theorem 4.10. For the remaining case, we have

$$
\begin{aligned}
h_{a_{\ell}}\left(x_{1}, \ldots, x_{\ell}\right) \cdot(1 \otimes 1) & \\
& =1 \otimes\left(P_{\ell}\left(h_{a_{\ell}}\left(x_{1}, \ldots, x_{\ell}\right)\right) \cdot 1\right)+x_{\ell} \otimes\left(\partial_{\ell}\left(h_{a_{\ell}}\left(x_{1}, \ldots, x_{\ell}\right)\right) \cdot 1\right) .
\end{aligned}
$$

By (2.6) all terms contain $h_{a_{\ell}}\left(x_{1}, \ldots, x_{\ell}\right)$ or $h_{a_{\ell}-1}\left(x_{1}, \ldots, x_{\ell+1}\right)$, which both lie in Ann $\mathrm{C}_{w_{k} z}$, and we are done.

It remains to prove that the polynomials (4.15) are a set of generators. Let $I$ be the ideal generated by them. We know that $C_{s_{j} w_{k} z}$ is a quotient of $R / I$. As for Lemma 2.2, the polynomials (4.15) are a basis of $I$. As for Proposition 2.3. the quotient $R / I$ has dimension $a_{1} \cdots a_{n}$. By Corollary 3.6 and an argument similar to the proof of Lemma 4.6, this coincides with the dimension of $\mathrm{C}_{s_{j} w_{k} z}$, and we are done.

4.3. Morphisms between Soergel modules. In each basis set (4.14) there is exactly one morphism of minimal degree, which we call the minimal degree morphism $\mathrm{C}_{w_{k} z} \rightarrow \mathrm{C}_{w_{k} z^{\prime}}$. For each $z \in D$, the vector space $\operatorname{Hom}_{R}\left(\mathrm{C}_{w_{k} z}, \mathrm{C}_{w_{k} z}\right)$ is a ring that is naturally isomorphic to $\mathrm{C}_{w_{k} z}$. Moreover, for $z, z^{\prime} \in D$ the vector space $\operatorname{Hom}_{R}\left(\mathrm{C}_{w_{k} z}, \mathrm{C}_{w_{k} z^{\prime}}\right)$ is naturally a $\left(\mathrm{C}_{w_{k} z^{\prime}}, \mathrm{C}_{w_{k} z}\right)$-bimodule. It follows directly from Corollary 4.11 that this bimodule is cyclic (even more, it is cyclic both as a left and as a right module), generated by the minimal degree morphism. In what follows, we will often refer to this fact saying that the minimal degree morphisms divide all other morphisms.

We let $D^{\prime}$ be the set of shortest coset representatives for $W_{k}^{\perp} \backslash \mathbb{S}_{n}$. In particular, for every $z \in D$ we have $z, w_{k} z \in D^{\prime}$.

Definition 4.13. For $z, z^{\prime} \in D$ we say that a morphism $C_{w_{k} z} \rightarrow C_{w_{k} z^{\prime}}$ is illicit if it factors through some $C_{y}$, where $y$ is a longest coset representative for $W_{k} \backslash \mathbb{S}_{n}$ with $y \notin D^{\prime}$.

The definition is motivated by the following. Let $\mathrm{W}_{z, z^{\prime}}$ be the subspace of $\operatorname{Hom}_{R}\left(\mathrm{C}_{w_{k} z}, \mathrm{C}_{w_{k} z^{\prime}}\right)$ consisting of all illicit morphisms; since it is a $\left(\mathrm{C}_{w_{k} z^{\prime}}, \mathrm{C}_{w_{k} z}\right)-$ submodule, we can define the quotient bimodule

$$
\mathrm{Z}_{z, z^{\prime}}=\operatorname{Hom}_{R}\left(\mathrm{C}_{w_{k} z}, \mathrm{C}_{w_{k} z^{\prime}}\right) / \mathrm{W}_{z, z^{\prime}} .
$$

This corresponds to the homomorphism space between projective modules in some parabolic category $\mathcal{O}$ (see Section [6). 
Lemma 4.14. Let $z, z^{\prime} \in D$, and suppose that for some index $j$ we have

$$
z_{i}^{\prime \vee}= \begin{cases}z_{i}^{\vee}+1 & \text { for } i=j, j+1, \\ z_{i}^{\vee} & \text { otherwise. }\end{cases}
$$

In particular $z^{\prime}=z s_{\ell} s_{\ell+1}$ for $\ell=j-z_{j}^{\vee}-1$, and the corresponding $\wedge \vee$-sequence in positions $\ell, \ell+1, \ell+2$ are

$$
z=\cdots \wedge \vee \vee \cdots \quad \text { and } \quad z^{\prime}=\cdots \vee \vee \wedge \cdots .
$$

Then $\mathrm{W}_{z, z^{\prime}}=\operatorname{Hom}_{R}\left(\mathrm{C}_{w_{k} z}, \mathrm{C}_{s_{k} z^{\prime}}\right)$ and $\mathrm{W}_{z^{\prime}, z}=\operatorname{Hom}_{R}\left(\mathrm{C}_{w_{k} z^{\prime}}, \mathrm{C}_{s_{k} z}\right)$.

Proof. It is enough to show that $\varphi \in \operatorname{Hom}_{R}\left(\mathrm{C}_{w_{k} z}, \mathrm{C}_{w_{k} z^{\prime}}\right), \varphi: 1 \mapsto x_{j} x_{j+1}$ and $\psi \in \operatorname{Hom}_{R}\left(\mathrm{C}_{w_{k} z^{\prime}}, \mathrm{C}_{w_{k} z}\right), \psi: 1 \mapsto 1$ are illicit, since they divide all other morphisms. First of all, note that by construction

$$
b_{i}^{z^{\prime}}= \begin{cases}b_{i}^{z}+1 & \text { for } i=\ell, \ell+1, \\ b_{i}^{z} & \text { otherwise. }\end{cases}
$$

Let $y=s_{j} z=z s_{\ell+1}$, and note that $y \notin D^{\prime}$. We know $\mathrm{C}_{w_{k} y}$ by Proposition 4.12 , Since $\operatorname{Ann}\left(C_{w_{k} z}\right) \subset \operatorname{Ann}\left(C_{w_{k} y}\right) \subset \operatorname{Ann}\left(C_{w_{k} z^{\prime}}\right)$, the morphism $\psi$ can be written as the composition of the natural quotient maps

$$
\mathrm{C}_{w_{k} z^{\prime}} \stackrel{1}{\longrightarrow} \mathrm{C}_{w_{k} y} \stackrel{1}{\longrightarrow} \mathrm{C}_{w_{k} z}
$$

hence it is illicit.

On the other side, $x_{\ell+1} \operatorname{Ann}\left(\mathrm{C}_{w_{k} z}\right) \subseteq \operatorname{Ann}\left(\mathrm{C}_{w_{k} y}\right)$ because by (2.3)

$$
x_{\ell+1} h_{b_{\ell+1}^{z}}\left(x_{1}, \ldots, x_{\ell+1}\right)=h_{b_{\ell+1}^{z}+1}\left(x_{1}, \ldots, x_{\ell+1}\right)-h_{b_{\ell+1}^{z}+1}\left(x_{1}, \ldots, x_{\ell}\right)
$$

and $h_{b_{\ell+1}^{z}+1}\left(x_{1}, \ldots, x_{\ell}\right) \in \operatorname{Ann}\left(C_{w_{k} y}\right)$ by the arguments of the proof of Lemma 2.7 Moreover, $x_{\ell} \operatorname{Ann}\left(\mathrm{C}_{w_{k} y}\right) \subseteq \operatorname{Ann}\left(\mathrm{C}_{w_{k} z^{\prime}}\right)$ because by (2.3) we have

$$
x_{\ell} h_{b_{\ell}^{z}}\left(x_{1}, \ldots, x_{\ell}\right)=h_{b_{\ell}^{z}+1}\left(x_{1}, \ldots, x_{\ell}\right)-h_{b_{\ell}^{z}+1}\left(x_{1}, \ldots, x_{\ell-1}\right),
$$

and this is in $\operatorname{Ann}\left(\mathrm{C}_{w_{k} z^{\prime}}\right)$ by Lemma2.7. Hence $\varphi$ can be written as the composition

$$
\mathrm{C}_{w_{k} z} \stackrel{x_{\ell+1}}{\longrightarrow} \mathrm{C}_{w_{k} y} \stackrel{x_{\ell}}{\longrightarrow} \mathrm{C}_{w_{k} z^{\prime}}
$$

and therefore is illicit.

Lemma 4.15. Let $z \in D$ and suppose $z_{j}^{\vee}=z_{j+1}^{\vee}$ for some index $j$. Let $\ell=j-z_{j}^{\vee}$, so that $s_{j} z=z s_{\ell}$. Then the endomorphism $1 \mapsto x_{\ell}$ of $\mathrm{C}_{w_{k} z}$ is illicit.

Proof. Let $y=s_{j} w_{k} z \notin D^{\prime}$. We claim that $x_{\ell} \operatorname{Ann}\left(\mathrm{C}_{w_{k} z}\right) \subseteq \operatorname{Ann}\left(\mathrm{C}_{y}\right)$ and hence that $1 \mapsto x_{\ell}$ defines a morphism $\mathrm{C}_{w_{k} z} \rightarrow \mathrm{C}_{y}$. By Theorem 4.10 and Proposition 4.12 the only thing to check is that $x_{\ell} h_{b_{\ell}^{z}}\left(x_{1}, \ldots, x_{\ell}\right) \in \operatorname{Ann}\left(\mathrm{C}_{y}\right)$. By (2.3) we have

$$
x_{\ell} h_{b_{\ell}^{z}}\left(x_{1}, \ldots, x_{\ell}\right)=h_{b_{\ell}^{z}+1}\left(x_{1}, \ldots, x_{\ell}\right)-h_{b_{\ell}^{z}+1}\left(x_{1}, \ldots, x_{\ell-1}\right) \in \operatorname{Ann}\left(C_{y}\right) .
$$

On the other side, again by Theorem 4.10 and Proposition 4.12, it is clear that $1 \mapsto 1$ defines a morphism $\mathrm{C}_{y} \rightarrow \mathrm{C}_{w_{k} z}$. Hence the endomorphism $1 \mapsto x_{\ell}$ of $\mathrm{C}_{w_{k} z}$ factors through $\mathrm{C}_{y}$ and is therefore illicit.

Lemma 4.16. Let $z \in D$. For every $j$ between $k+1$ and $n-1$ the morphism

$$
1 \longmapsto x_{\ell} x_{\ell+1} \cdots x_{\ell^{\prime}}
$$

where $\ell=j-z_{j}^{\vee}$ and $\ell^{\prime}=(j+1)-z_{j+1}^{\vee}-1$, is illicit. 
Proof. Let $y \in D$ be defined by $y_{i}^{\vee}=z_{i}^{\vee}$ for $i \neq j$, while $y_{j}^{\vee}=z_{j+1}^{\vee}$. From Corollary 4.11 we have that $1 \mapsto 1$ and $1 \mapsto x_{\ell} x_{\ell+1} \cdots x_{\ell^{\prime}-1}$ define morphisms $\mathrm{C}_{w_{k} z} \rightarrow \mathrm{C}_{w_{k} y}$ and $\mathrm{C}_{w_{k} y} \rightarrow \mathrm{C}_{w_{k} z}$ respectively. By Lemma 4.15 the endomorphism $1 \rightarrow x_{\ell^{\prime}}$ of $\mathrm{C}_{w_{k} y}$ is illicit, and so is (4.26), since it can be expressed as the composition of these three morphisms.

Theorem 4.17. For all $z, z^{\prime} \in D$ define a subbimodule $\widetilde{\mathbf{W}}_{z, z^{\prime}}$ of the homomorphism space $\operatorname{Hom}_{R}\left(\mathrm{C}_{w_{k} z}, \mathrm{C}_{w_{k} z^{\prime}}\right)$ as follows:

(i) if for some index $1 \leq j \leq n-k-1$ we have $\vee_{j}^{z} \geq \vee_{j+1}^{z^{\prime}}$ or $\vee_{j}^{z^{\prime}} \geq \vee_{j+1}^{z}$, then we set $\widetilde{\mathrm{W}}_{z, z^{\prime}}=\operatorname{Hom}\left(\mathrm{C}_{w_{k} z}, \mathrm{C}_{w_{k} z^{\prime}}\right)$;

(ii) otherwise we define $\widetilde{\mathrm{W}}_{z, z^{\prime}}$ to be the subbimodule generated by the morphisms

$$
1 \mapsto\left(x_{\vee_{j}^{z}} x_{\vee_{j}^{z}+1} \cdots x_{\beta(j)}\right)\left(x_{1}^{c_{1}} \cdots x_{n-1}^{c_{n-1}}\right) \quad \text { for } 1 \leq j \leq n-k
$$

where $c_{i}=\max \left\{b_{i}^{z^{\prime}}-b_{i}^{z}, 0\right\}$ and

$$
\beta(j)= \begin{cases}\min \left\{\vee_{j+1}^{z}, \vee_{j+1}^{z^{\prime}}\right\}-1 & \text { if } j<n-k, \\ n & \text { if } j=n-k .\end{cases}
$$

Then we have $\widetilde{\mathrm{W}}_{z, z^{\prime}}=\mathrm{W}_{z, z^{\prime}}$.

Example 4.18. Let us consider the following example:

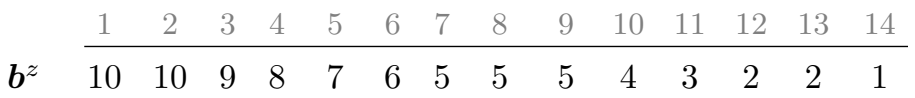

$$
\begin{aligned}
& z \wedge V_{1} \wedge \wedge \wedge \wedge \wedge V_{2} \bar{V}_{3} \wedge \wedge \wedge 1 \bar{V}_{4} \wedge \\
& z^{\prime} \vee_{1} \wedge \wedge \wedge \vee_{2} \wedge \wedge \wedge \wedge \wedge \vee_{3} \wedge \wedge\left(\bar{V}_{4}^{\prime} ;\right.
\end{aligned}
$$

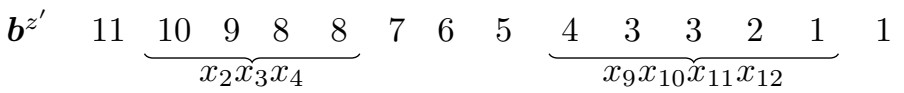

For convenience we have written the subscripts of the $\vee^{\prime}$ 's, indicating their progressive number. We are in case (ii) and the generating morphisms (4.27) of $\widetilde{W}_{z, z^{\prime}}$ are

\begin{tabular}{l|l}
$j$ & morphism \\
\hline 1 & $1 \longmapsto\left(x_{2} x_{3} x_{4}\right)\left(x_{1} x_{5} x_{6} x_{7}\right)$ \\
2 & $1 \longmapsto\left(x_{8}\right)\left(x_{1} x_{5} x_{6} x_{7}\right)$ \\
3 & $1 \longmapsto\left(x_{9} x_{10} x_{11} x_{12}\right)\left(x_{1} x_{5} x_{6} x_{7}\right)$ \\
4 & $1 \longmapsto\left(x_{13}\right)\left(x_{1} x_{5} x_{6} x_{7}\right)$
\end{tabular}

In the picture, the case $j=1$ is highlighted in solid and the case $j=3$ is highlighted in dashed.

Proof of Theorem 4.17. First, we assume that $\vee_{j}^{z} \geq \vee_{j+1}^{z^{\prime}}$ for some index $1 \leq j<$ $n-k$. Pick $j$ minimal with this property. Notice that by the minimality of $j$ we have $\vee_{j-1}^{z}<\vee_{j}^{z^{\prime}}$ (if $j>1$ ), and hence on the left of the $j$-th $\vee$ of $z$ there is a $\wedge$ (this remains true also if $j=1$, since in this case $\vee_{1}^{z} \geq \vee_{2}^{z^{\prime}}>\vee_{1}^{z^{\prime}} \geq 1$ ). Let $\alpha=\vee_{j}^{z}$ 
and $\ell=\vee_{j+1}^{z}-\vee_{j}^{z}$, and define $z^{(1)}$ and $z^{(2)}$ as

$$
\begin{aligned}
z & \\
z^{(1)} & =z s_{\alpha+\ell-1} s_{\alpha+\ell-2} \cdots s_{\alpha+1} \\
z^{(2)} & =z^{(1)} s_{\alpha-1} s_{h}
\end{aligned}
$$

$$
\begin{aligned}
& \wedge \vee \overbrace{\wedge \cdots \wedge \vee}^{\ell}, \\
& \wedge \vee \vee \cdots \wedge \wedge, \\
& \vee \vee \wedge \cdots \wedge \wedge,
\end{aligned}
$$

where on the right we pictured the corresponding $\wedge \vee$-sequences between positions $\alpha-1$ and $\alpha+\ell$ (and we included $z$ for clarity). The composition

$$
\mathrm{C}_{w_{k} z} \stackrel{1}{\longrightarrow} \mathrm{C}_{w_{k} z^{(1)}} \stackrel{x_{\ell-1} x_{\ell}}{\longrightarrow} \mathrm{C}_{w_{k} z^{(2)}}
$$

is illicit by Lemma 4.14. Composing with the minimal degree morphism $C_{w_{k} z^{(2)}} \rightarrow$ $\mathrm{C}_{w_{k} z^{\prime}}$ we obtain the minimal degree morphism $\mathrm{C}_{w_{k} z} \rightarrow \mathrm{C}_{w_{k} z^{\prime}}$, which is therefore illicit. It follows that $\operatorname{Hom}_{R}\left(\mathrm{C}_{w_{k} z}, \mathrm{C}_{w_{k} z^{\prime}}\right)=\mathrm{W}_{z, z^{\prime}}$.

A straightforward dual argument (cf. \$2.4) proves that $\operatorname{Hom}_{R}\left(\mathrm{C}_{w_{k} z^{\prime}}, \mathrm{C}_{w_{k} z}\right)=$ $\mathrm{W}_{z^{\prime}, z}$. Swapping $z$ and $z^{\prime}$ it follows that $\operatorname{Hom}_{R}\left(\mathrm{C}_{w_{k} z}, \mathrm{C}_{s_{k} z^{\prime}}\right)=\mathrm{W}_{z, z^{\prime}}$ if $\vee_{j}^{z^{\prime}} \geq \vee_{j+1}^{z}$.

Now assume we are in case (ii) and fix an index $j$. First, let us consider the case $\vee_{j+1}^{z^{\prime}}<\bigvee_{j+1}^{z}$, so that $\beta(j)=\bigvee_{j+1}^{z^{\prime}}$. Let $\gamma=\bigvee_{j}^{z}, \delta=\bigvee_{j+1}^{z^{\prime}}, \varepsilon=\bigvee_{j+1}^{z}$. Define $z^{(1)}$, $z^{(2)}$ and $z^{(3)}$ by

$$
\begin{aligned}
& \text { z } \\
& z^{(1)}=z s_{\gamma} s_{\gamma+1} \cdots s_{\delta-1} \\
& \vee \wedge \cdots \wedge \wedge \cdots \wedge \vee \\
& z^{(2)}=z^{(1)} s_{\varepsilon-1} s_{\varepsilon-2} \cdots s_{\delta+1} \\
& \wedge \cdots \wedge \vee \wedge \cdots \wedge \vee \\
& z^{(3)}=z^{(2)} s_{\delta-1} s_{\delta} \\
& \wedge \cdots \wedge \vee \vee \wedge \cdots \wedge \text {, } \\
& \wedge \cdots \vee \vee \wedge \wedge \cdots \wedge,
\end{aligned}
$$

where on the right we pictured the corresponding $\wedge \vee$-sequences between positions $\gamma$ and $\varepsilon$. The composition

$$
\mathrm{C}_{w_{k} z} \stackrel{1}{\rightarrow} \mathrm{C}_{w_{k} z^{(1)}} \stackrel{x_{\delta+1} x_{\delta+2} \cdots x_{\varepsilon-1}}{\longrightarrow} \mathrm{C}_{w_{k} z^{(2)}} \stackrel{x_{\delta-1} x_{\delta}}{\longrightarrow} \mathrm{C}_{w_{k} z^{(3)}}
$$

is illicit by Lemma 4.14. By construction, the composition of (4.37) with the minimal degree morphism $C_{w_{k} z^{(3)}} \rightarrow C_{w_{k} z^{\prime}}$ equals the morphism (4.27) from $C_{w_{k} z}$ to $\mathrm{C}_{w_{k} z^{\prime}}$, which is therefore illicit.

Let us now consider the other case $\vee_{j+1}^{z} \leq \vee_{j+1}^{z^{\prime}}$. By Lemma 4.16 the endomorphism of $\mathrm{C}_{w_{k} z}$ defined by

$$
1 \mapsto x_{\vee_{j}^{z}} x_{\vee_{j}^{z}+1} \cdots x_{\vee_{j+1}^{z}}
$$

is illicit. This morphism divides the morphism (4.27), which is therefore illicit.

To conclude the proof we are left to check that in case (ii) $\mathrm{W}_{z, z^{\prime}} \subseteq \widetilde{\mathrm{W}}_{z, z^{\prime}}$. Unfortunately we cannot check this directly. Instead, by Lemma 5.9 in the next section we have that the dimensions of the quotients of $\operatorname{Hom}_{R}\left(\mathrm{C}_{w_{k} z}, \mathrm{C}_{z_{k} z^{\prime}}\right)$ by $\mathrm{W}_{z, z^{\prime}}$ and $\widetilde{W}_{z, z^{\prime}}$ agree. This implies that $\mathrm{W}_{z, z^{\prime}}=\widetilde{\mathrm{W}}_{z, z^{\prime}}$.

4.4. Grading. In order to keep the computations more transparent, we decided to postpone the introduction of the grading until now. The ring $R$ is graded with $\operatorname{deg} x_{i}=2$. Since the ideal $J_{n}$ is homogeneous, $B$ is also graded, and the graded definition of the module $B_{i}$ is $B_{i}=B \otimes_{i} B\langle-1\rangle$. By Soergel theorems all $C_{w}$ for 
$w \in \mathbb{S}_{n}$ are graded. In the graded version of the module $\mathbf{C}_{w_{k} z}$ the cyclic generator is in degree $-\ell\left(w_{k} z\right)$. Then (4.1) has the following graded version:

$$
\operatorname{gr} \operatorname{dim}_{\mathbb{C}} C_{z}=v^{-\ell\left(w_{k} z\right)} \sum_{w^{\prime} \preceq z} \mathcal{P}_{w^{\prime}, z}\left(v^{2}\right) .
$$

The spaces $\mathrm{W}_{z, z^{\prime}}$ are homogeneous submodules, and the quotients $Z_{z, z^{\prime}}$ are then graded modules.

By our discussion in 2.4 and with the opportune degree shifting we put on the modules $\mathrm{C}_{w_{k} z}$, it follows that all modules $\mathrm{C}_{w_{k} z}$ are graded self-dual. In particular

$$
\operatorname{Hom}_{R}\left(\mathrm{C}_{w_{k} z}, \mathrm{C}_{w_{k} z^{\prime}}\right) \cong \operatorname{Hom}_{R}\left(\mathrm{C}_{w_{k} z^{\prime}}, \mathrm{C}_{w_{k} z}\right)
$$

is an isomorphism of graded vector spaces for all $z, z^{\prime} \in D$. The explicit isomorphism was described in (2.19).

\section{The DiAgram ALGEBRA}

We now want to define some diagram algebras over $\mathbb{C}$, analogous to the generalized Khovanov algebras defined in BS11a. We will use some diagrams which represent morphisms between the Soergel modules we studied in the previous section.

We point out that the major difficulty is the definition of the multiplication of two diagrams, which is not simply stacking one on top of the other (as in many other diagram algebras), but instead a quite involved process. Brundan and Stroppel use Khovanov's TQFT to define this multiplication. Since there is not an analogue of such a TQFT in our case, we construct the multiplication in an indirect way using composition of morphisms between Soergel modules. A drawback of our definition of the multiplication is that it is not clear how one can define diagrammatically bimodules for the diagram algebra, as in [BS10].

Using the same techniques as BS11a we will describe explicitly the graded cellular structure $(\$ 5.3)$ and the graded properly stratified structure $(\$ 5.4)$.

5.1. Diagrams. We start introducing the diagrams on which our algebras will be built. We will redefine some keywords that are commonly used in Lie theory (such as weight and block) in a diagrammatic sense.

Weights. A number line $\mathbf{L}$ is a horizontal line containing a finite number of vertices indexed by a set of consecutive integers in increasing order from left to right. Given a number line, a weight is obtained by labeling each of the vertices by $\wedge$ or $\vee$.

On the set of weights there is the partial order called Bruhat order, generated by $\wedge \vee \succ \vee \wedge$. For weights $\lambda, \mu$ declare $\lambda \sim \mu$ if $\mu$ can be obtained from $\lambda$ by permuting $\Lambda$ 's and $\vee$ 's.

Blocks. A block $\Gamma$ is a $\sim$-equivalence class of weights. From now on, let us fix a block $\Gamma$. Also let $k$ be the number of $\wedge$ 's and $n-k$ be the number of $\vee$ 's of any weight of $\Gamma$. The weights of $\Gamma$ can be identified with $\wedge \vee$-sequences in the sense of $\$ 3.2$, and hence with elements of $D_{n, k}$. For a weight $\lambda$, we can then define as in $\$ 3.2$ the position sequences $\left(\wedge_{1}^{\lambda}, \ldots, \wedge_{k}^{\lambda}\right)$ and $\left(\vee_{1}^{\lambda}, \ldots, \vee_{n-k}^{\lambda}\right)$ and the $\boldsymbol{b}$-sequence $\boldsymbol{b}^{\lambda}$. 
Enhanced weights. An enhanced weight $\lambda^{\sigma}$ is a weight $\lambda$ together with a bijection $\sigma$ between the vertices labeled $\wedge$ in $\lambda$ and the set $\{1, \ldots, k\}$. By numbering the $\Lambda$ 's from the left to the right we may view $\sigma$ as an element in $\mathbb{S}_{k}$ and call it the underlying permutation. We call $\lambda$ the underlying weight. We will also say that we obtain the enhanced weight $\lambda^{\sigma}$ by enhancing the weight $\lambda$ with the permutation $\sigma$. Notice that there are exactly $k$ ! enhanced weights with the same underlying weight.

We define a partial order on the set of enhanced weights by the following rule:

$$
\lambda^{\sigma} \preceq \mu^{\tau} \Longleftrightarrow \lambda \prec \mu \text { or }(\lambda=\mu \text { and } \ell(\sigma) \leq \ell(\tau)) .
$$

Fork diagrams. An $m$-fork is a tree with a unique branching point (the root) of valency $m$; the other $m$ vertices of the tree are called the leaves. A 1-fork will also be called a ray. This is an example of a 5 -fork:

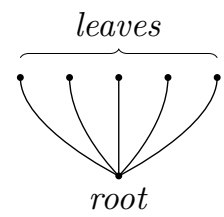

Let $\mathbf{V}$ be the set of vertices of the number line $\mathbf{L}$, and let $\mathbf{H}_{-}\left(\right.$resp. $\left.\mathbf{H}_{+}\right)$be the half-plane below (resp. above) L. A lower fork diagram is a diagram made by the number line $\mathbf{L}$ together with some forks contained in $\mathbf{H}_{-}$, such that the leaves of each $m$-fork are $m$ distinct consecutive vertices in $\mathbf{V}$; we require each vertex of $\mathbf{V}$ to be a leaf of some fork. The forks and rays of a lower fork diagram will also be called lower forks and lower rays.

Upper rays, upper forks and upper fork diagrams are defined in an analogous way. If $c$ is a lower fork diagram, the mirror image $c^{*}$ through the horizontal number line is an upper fork diagram, and vice versa. The following are examples of a lower fork diagram $c$ and its mirror image $c^{*}$ :
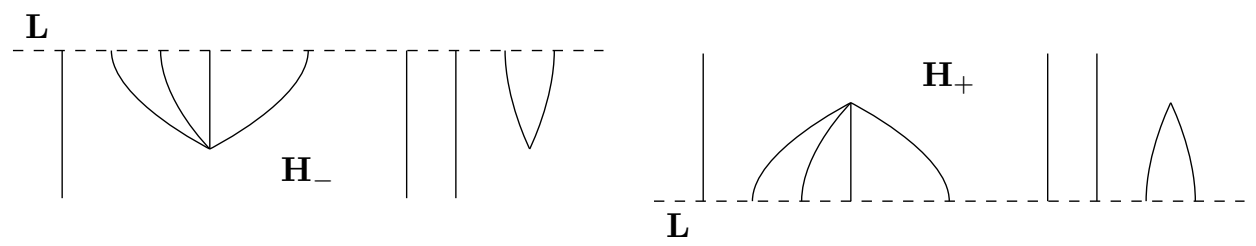

Oriented diagrams. If $c$ is a lower fork diagram and $\lambda$ is a weight with the same underlying number line, we can glue them to obtain a diagram $c \lambda$. We call $c \lambda$ an unenhanced oriented lower fork diagram if:

- each $m$-fork for $m \geq 1$ is labeled with exactly one $\vee$ and $m-1 \wedge$ 's;

- the diagram begins at the left with a (possibly empty) sequence of rays labeled $\wedge$, and there are no other rays labeled $\wedge$ in $c$.

Notice that by definition each $\wedge$ and $\vee$ of $\lambda$ labels some fork of $c$. Analogously, we call $\mu d$ an unenhanced oriented upper fork diagram if $d^{*} \mu$ is an unenhanced oriented lower fork diagram. The orientation of an unenhanced oriented lower (or upper) fork diagram is the corresponding weight.

An (enhanced) oriented lower fork diagram $c \lambda^{\sigma}$ is an unenhanced oriented lower fork diagram $c \lambda$ together with a permutation $\sigma \in \mathbb{S}_{k}$ such that $\lambda^{\sigma}$ is an enhanced 
weight. Similarly we define an (enhanced) oriented upper fork diagram. If not explicitly specified, our oriented lower/upper fork diagrams will always be enhanced.

For $m \geq 1$ and $1 \leq i \leq m$ we define $\lambda(m, i)$ to be the weight formed by one $\vee$ and $m-1 \wedge$ 's, where the $\vee$ is at the $i$-th place. Note that a lower fork diagram $c$ consisting of only a lower $m$-fork admits exactly $m$ ! orientations, and they are exactly the $\lambda(m, i)^{\sigma}$ for $i \in\{1, \ldots, m\}, \sigma \in \mathbb{S}_{m-1}$.

By a fork diagram we mean a diagram of the form $a b$ obtained by gluing a lower fork diagram $a$ underneath an upper fork diagram $b$, assuming that they have the same underlying number lines. An unenhanced oriented fork diagram is a fork diagram $a \lambda b$ obtained by gluing an oriented lower fork diagram $a \lambda$ and an oriented upper fork diagram $\lambda b$, as in the picture:

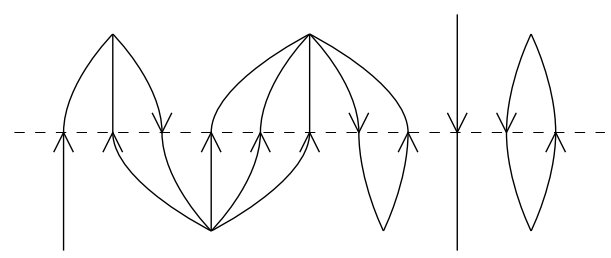

An (enhanced) oriented fork diagram is obtained by additionally enhancing the corresponding weight.

Degrees. Define the degree of an unenhanced oriented lower (or upper) $m$-fork by setting $\operatorname{deg}(c \lambda(m, i))=\operatorname{deg}\left(\lambda(m, i) c^{*}\right)=(i-1)$. Define then the degree of an unenhanced oriented lower (resp. upper) fork diagram to be the sum of the degrees of all the lower (resp. upper) forks. Finally, the degree of an unenhanced oriented fork diagram $a \lambda b$ is

$$
\operatorname{deg}(a \lambda b)=\operatorname{deg}(a \lambda)+\operatorname{deg}(\lambda b) .
$$

Moreover, define the degree of a permutation $\sigma$ as $\operatorname{deg}(\sigma)=2 \ell(\sigma)$. Then we define the degree of enhanced oriented diagrams by

$$
\begin{aligned}
\operatorname{deg}\left(a \lambda^{\sigma}\right) & =\operatorname{deg}(a \lambda)+\operatorname{deg}(\sigma), \\
\operatorname{deg}\left(\lambda^{\sigma} b\right) & =\operatorname{deg}(\lambda b)+\operatorname{deg}(\sigma), \\
\operatorname{deg}\left(a \lambda^{\sigma} b\right) & =\operatorname{deg}(a \lambda b)+\operatorname{deg}(\sigma)=\operatorname{deg}(a \lambda)+\operatorname{deg}(\lambda b)+\operatorname{deg}(\sigma) .
\end{aligned}
$$

In particular, enhancing with the neutral element $e \in \mathbb{S}_{k}$ preserves the degree.

Example 5.1. Consider the fork diagram $a \lambda b$ given by:

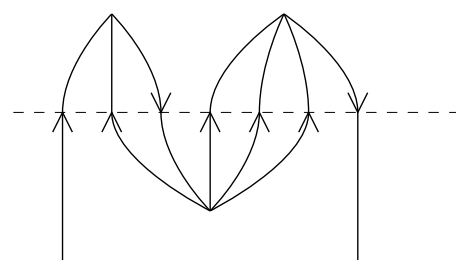

We have $\operatorname{deg}(a \lambda)=1$ and $\operatorname{deg}(\lambda b)=2+3=5$, so that $\operatorname{deg}(a \lambda b)=6$. We can enhance the diagram with any permutation $\sigma \in \mathbb{S}_{5}$, and then $\operatorname{deg}\left(a \lambda^{\sigma} b\right)=6+2 \ell(\sigma)$. 
The lower fork diagram associated to a weight. There is a natural way to associate a lower fork diagram to a weight $\lambda$ :

Lemma 5.2. For each weight $\lambda$ there is a unique lower fork diagram, denoted $\underline{\lambda}$, such that $\underline{\lambda} \lambda^{e}$ is an oriented lower fork diagram of degree 0 .

Proof. Suppose that some oriented lower fork diagram $c \lambda^{e}$ of degree 0 exists. Recall that, by the definition of orientation, each fork of $c$ is labeled by at most one $V$ of $\lambda$; by the assumption on the degree, this $\vee$ has to be the leftmost label of the corresponding fork. As a consequence, each $m$-fork of $c$, with the exception only of some initial rays labeled by $\wedge$, has to be labeled by the weight $\lambda(m, 1)$. In other words, the lower fork diagram $c$ is obtained in the following way: examine the weight $\lambda$ from the left to the right and find all maximal subsequences consisting of a $\vee$ followed by some (eventually empty) set of $\Lambda$ 's; draw a lower fork under each of these subsequences, and then draw lower rays under the remaining $\wedge$ 's which are at the beginning of $\lambda$. Hence $c$ exists and is uniquely determined.

Analogously we let $\bar{\lambda}=(\underline{\lambda})^{*}$ be the unique upper fork diagram such that $\lambda^{e} \bar{\lambda}$ is an oriented upper fork diagram of degree 0 .

Example 5.3. As an example, let us illustrate the procedure of constructing $\underline{\lambda}$ for $\lambda=\wedge \wedge \vee \wedge \wedge \wedge \vee \vee \wedge \vee$. First, we circle all maximal subsequences consisting of a $\vee$ followed by $\wedge$ 's:

$$
\wedge \wedge \vee \wedge \wedge \wedge \vee \vee \wedge \vee
$$

Then we draw a lower fork under each of such subsequences and lower rays under the remaining $\wedge$ 's at the beginning of $\lambda$ :

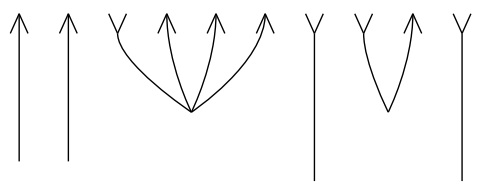

The resulting lower fork diagram is then

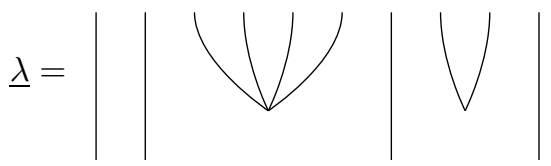

For weights $\mu$ and $\lambda$, we use the notation $\mu \subset \lambda$ to indicate that $\mu \sim \lambda$ and $\mu \lambda^{e}$ is an oriented lower fork diagram.

Lemma 5.4. Let $\lambda, \mu$ be two weights in the same block $\Gamma$. If $\underline{\lambda}=\mu$, then $\lambda=\mu$. If $\underline{\mu} \lambda$ is oriented, then $\mu \preceq \lambda$ in the Bruhat order.

Proof. Being in the same block, the weights $\lambda$ and $\mu$ have the same number of $\wedge$ 's and V's; let $h$ be the number of V's. Consider the $h$ rightmost forks of $\underline{\lambda}$ and let $a_{1}, \ldots, a_{h}$ be their initial positions; then $\lambda$ is uniquely determined by the condition of having $\vee$ 's in the positions $a_{1}, \ldots, a_{h}$ and $\wedge$ 's elsewhere. Hence the first claim follows.

Now, given the lower fork diagram $\mu$, let $F_{1}, \ldots, F_{h}$ denote its $h$ rightmost forks. Let also $\Gamma_{\mu}=\{\lambda \in \Gamma \mid \underline{\mu} \lambda$ is oriented $\}$. Then $\lambda \in \Gamma_{\mu}$ if and only if each $\vee$ of $\lambda$ labels exactly one of the $F_{i}$ 's. Since $\mu$ is the weight of $\Gamma_{\mu}$ with the $\vee$ 's in the leftmost positions, it follows that $\mu$ is the minimal element in $\Gamma_{\mu}$ with respect to the Bruhat order. 
In particular, given our fixed block $\Gamma$, it follows that every lower fork diagram $a$ (such that $a \mu$ is oriented for some $\mu \in \Gamma$ ) determines a unique weight $\lambda$ with $\underline{\lambda}=a$. In what follows, we will sometime interchange $a$ and $\lambda$ in the notation: for example, we will write $\vee_{j}^{a}$ for $\vee_{j}^{\lambda}$ or $\boldsymbol{b}^{a}$ for $\boldsymbol{b}^{\lambda}$ and so on.

We now collect some lemmas that we will need later.

Lemma 5.5. Let $\lambda, \mu$ be two weights in the same block $\Gamma$.

(i) The lower fork diagram $\underline{\lambda} \mu$ is oriented if and only if

$$
\vee_{i}^{\lambda} \leq \vee_{i}^{\mu}<\vee_{i+1}^{\lambda} \quad \text { for all } i \in 1, \ldots, n-k-1 .
$$

(ii) There exists an oriented fork diagram $\underline{\lambda} \eta \bar{\mu}$ for some $\eta \in \Gamma$ if and only if

$$
\vee_{i}^{\lambda}<\vee_{i+1}^{\mu} \quad \text { and } \quad \vee_{i}^{\mu}<\vee_{i+1}^{\lambda} \quad \text { for all } i \in 1, \ldots, n-k-1 \text {. }
$$

Proof. It is clear that (ii) follows from (i) so let us prove(i), It is easy to see that the lower fork diagram $\underline{\lambda} \mu$ is oriented if and only if each lower fork of $\underline{\lambda}$ is labeled by exactly one $\vee$ : this is exactly the same as (5.6).

Lemma 5.6. Consider weights $\lambda, \mu \in \Gamma$ with the corresponding $\boldsymbol{b}$-sequences $\boldsymbol{b}^{\lambda}, \boldsymbol{b}^{\mu}$.

(a) If $\mu \succeq \lambda$, then $b_{i}^{\mu} \leq b_{i}^{\lambda}$ for all $i=1, \ldots, n$.

(b) If $\underline{\lambda} \mu$ is oriented, then $b_{i}^{\lambda}-b_{i}^{\mu} \leq 1$ for all $i=1, \ldots, n$.

(c) If $\underline{\lambda} \eta^{e} \bar{\mu}$ is oriented (for some weight $\eta \in \Gamma$ ), then $\left|b_{i}^{\lambda}-b_{i}^{\mu}\right| \leq 1$ for all $i=1, \ldots, n$.

Proof. If $\mu \succeq \lambda$, then the $i$-th $\vee$ of $\mu$ is not on the right of the $i$-th $\vee$ of $\lambda$, and the first claim follows.

Let $\underline{\lambda} \mu$ be oriented. By Lemma 5.5 we have $\vee_{i}^{\lambda} \leq \vee_{i}^{\mu}<\vee_{i+1}^{\lambda}$. This means that for every vertex $v \in \mathbf{V}$ there is at most one $\wedge$ more to the right of $v$ in $\lambda$ than in $\mu$. This is exactly (b)

The last claim follows from the second: if $\lambda \eta^{\sigma} \bar{\mu}$ is oriented (for some weight $\eta$ with $\boldsymbol{b}$-sequence $\left.\boldsymbol{b}^{\eta}\right)$, then $b_{i}^{\lambda}-b_{i}^{\mu}=b_{i}^{\lambda}-b_{i}^{\eta}+b_{i}^{\eta}-b_{i}^{\mu} \in\{1-1,1+0,0-1,0+0\}$.

Since we have identified $\Gamma$ with $D_{n, k}$, we can define the length $\ell(\lambda)$ of any weight $\lambda \in \Gamma$ to be the length of the corresponding permutation in $D_{n, k}$.

Lemma 5.7. Consider weights $\lambda, \eta$ in the same block $\Gamma$. Then

$$
\operatorname{deg}\left(\underline{\lambda} \eta^{\sigma}\right)=\ell(\lambda)-\ell(\eta)+2 \ell(\sigma)
$$

Proof. Since $\underline{\lambda} \eta$ is oriented, the weight $\eta$ is obtained from $\lambda$ permuting the $\wedge$ 's and $\checkmark$ 's on each lower fork of $\underline{\lambda}$. The degree of $\underline{\lambda} \eta$ is the sum of how much each $\vee$ of $\lambda$ has been moved to the right to reach the corresponding $V$ of $\eta$; hence it is just the length of this permutation. In other words, if we let $z, z^{\prime} \in D_{n, k}$ be the permutations corresponding to $\lambda, \eta$ respectively, then we have $z=z^{\prime} y$ for some $y \in \mathbb{S}_{n}$ with $\ell\left(z^{\prime}\right)=\ell(z)+\ell(y)$, and $\operatorname{deg}(\underline{\lambda} \eta)=\ell(y)$.

5.2. The algebra structure. We connect now our diagrams with the commutative algebra from Section 4. Let us fix a block $\Gamma$ with $k \wedge$ 's and $n-k \vee$ 's. 
Relations with polynomial rings. We associate to the weight $\lambda$ the $\operatorname{ring} R_{\lambda}=R_{b^{\lambda}}=$ $R / I_{b^{\lambda}}$ (defined in 2.2), and we want to describe $Z_{z, z^{\prime}}$ from (4.17) diagrammatically.

Given an oriented lower fork diagram $\underline{\lambda} \eta^{\sigma}$, we define the polynomial

$$
p_{\underline{\lambda} \eta^{\sigma}}=\mathfrak{S}_{\sigma}^{\prime}\left(x_{\wedge_{1}^{\eta}}, \ldots, x_{\wedge_{k}^{\eta}}\right) \cdot \prod_{j=1}^{n-k} x_{\vee_{j}^{\lambda}} x_{\vee_{j}^{\lambda}+1} \cdots x_{\vee_{j}^{\eta}-1} \in R
$$

with $\mathfrak{S}_{\sigma}^{\prime}\left(x_{\wedge_{1}^{\eta}}, \ldots, x_{\wedge_{k}^{\eta}}\right)$ as defined in $\$ 2.5$. Notice that the terms on the right always make sense because, since $\underline{\lambda} \eta^{\sigma}$ is oriented, $\vee_{j}^{\eta} \geq \vee_{j}^{\lambda}$ for all indices $j$ (cf. Lemma 5.5). Often we will consider $p_{\underline{\lambda} \varepsilon^{\sigma}}$ as a polynomial in the quotient $R_{\lambda}$, but it will be convenient to have a chosen lift in $R$. Notice that we have

$$
\operatorname{deg}\left(p_{\underline{\lambda} \eta^{\sigma}}\right)=2(\ell(\sigma)+\ell(\lambda)-\ell(\eta)) .
$$

Proposition 5.8. Let $\lambda, \mu \in \Gamma$ be weights, and let $z, z^{\prime}$ be the corresponding elements of $D$. Let $\mathcal{Z}_{\mu, \lambda}$ be the graded vector space with homogeneous basis

$$
\left\{\underline{\mu} \eta^{\sigma} \bar{\lambda} \mid \underline{\mu} \eta^{\sigma} \bar{\lambda} \text { is an oriented fork diagram }\right\} \text {. }
$$

With $\widetilde{\mathrm{W}}_{z, z^{\prime}}$ as defined in Theorem 4.17 we have an isomorphism of graded vector spaces

$$
\begin{aligned}
\Psi: \mathcal{Z}_{\mu, \lambda} & \longrightarrow \operatorname{Hom}_{R}\left(\mathrm{C}_{w_{k} z}, \mathrm{C}_{w_{k} z^{\prime}}\right) / \widetilde{\mathrm{W}}_{z, z^{\prime}}, \\
\underline{\mu} \eta^{\sigma} \bar{\lambda} \longmapsto & \left(1 \mapsto p_{\underline{\mu} \eta^{\sigma}}\right)+\widetilde{\mathrm{W}}_{z, z^{\prime}} .
\end{aligned}
$$

Proof. First, note that $p_{\underline{\mu} \eta^{\sigma}}=p_{\underline{\mu} \eta^{e}} \mathfrak{S}_{\sigma}^{\prime}\left(x_{\wedge_{1}^{\eta}}, \ldots, x_{\wedge_{k}^{\eta}}\right)$. By definition we have $p_{\underline{\mu} \eta^{e}}=$ $x_{1}^{\varepsilon_{1}} \cdots x_{n}^{\varepsilon_{n}}$ with $\varepsilon_{j}=b_{j}^{\mu}-b_{j}^{\eta}$. By Lemma 5.6. $b_{j}^{\lambda} \geq b_{j}^{\eta}$ for every $j$; hence $\varepsilon_{j} \geq$ $b_{j}^{\mu}-b_{j}^{\lambda}$. By Corollary 4.11, the map $1 \mapsto p_{\mu \eta^{\sigma}}$ induces a well-defined morphism in $\operatorname{Hom}_{R}\left(\mathrm{C}_{w_{k} z}, \mathrm{C}_{w_{k} z^{\prime}}\right)$, hence also in the quotient.

Let us show that (5.12) is homogeneous of degree 0 . The degree of the morphism $1 \mapsto p_{\mu \eta^{\sigma}}$ in $\operatorname{Hom}_{R}\left(\mathrm{C}_{w_{k} z}, \mathrm{C}_{w_{k} z^{\prime}}\right)$ is $\operatorname{deg}\left(p_{\mu \eta^{\sigma}}\right)-\ell\left(w_{k} z^{\prime}\right)+\ell\left(w_{k} z\right)$, that is, the same as $\operatorname{deg}\left(p_{\underline{\mu} \eta^{\sigma}}\right)-\ell\left(z^{\prime}\right)+\ell(z)=\operatorname{deg}\left(p_{\underline{\mu} \eta^{\sigma}}\right)-\bar{\ell}(\mu)+\ell(\lambda)$. By (5.10) this is $\ell(\lambda)+\ell(\mu)-$ $2 \ell(\eta)+2 \ell(\sigma)$. By Lemma 5.7, this is the same as $\operatorname{deg}\left(\mu \eta^{\sigma} \bar{\lambda}\right)$.

Next, we want to see that $p_{\mu \eta^{\sigma}}$ is always a monomial of the basis (4.14). For that, note that by definition $\varepsilon_{j}=1$ exactly when $b_{j}^{\mu}=b_{j}^{\eta}+1$. Moreover, the monomial $\mathfrak{S}_{\sigma}^{\prime}\left(x_{\wedge_{1}^{\eta}}, \ldots, x_{\wedge_{k}^{\eta}}\right)=x_{1}^{i_{1}} \cdots x_{n}^{i_{n}}$ is by construction in the basis of $R_{\eta}$; that means that $i_{j}<b_{j}^{\eta}$ for every $j$. It follows that $i_{j}+\varepsilon_{j}<b_{j}^{\mu}$; hence $p_{\mu} \eta^{\sigma}$ is a monomial of the basis (4.14).

We claim now that none of the $p_{\mu \eta^{\sigma}}$ is in $\widetilde{\mathrm{W}}_{z, z^{\prime}}$. Note that by construction the indeterminate $x_{\vee_{j}^{\eta}}$ does not appear in $p_{\underline{\mu} \eta^{\sigma}}$. By Lemma 5.5 we have $\vee_{j}^{\lambda} \leq \vee_{j}^{\eta}$ and both $\vee_{j}^{\eta}<\vee_{j+1}^{\lambda}$ and $\vee_{j}^{\eta}<\vee_{j+1}^{\mu}$. This means that both $x_{\vee_{j}^{\lambda}} \cdots x_{\vee_{j+1}^{\lambda}-1}$ and $x_{\vee_{j}^{\lambda}} \cdots x_{\vee_{j+1}^{\mu}-1}$ do not divide $p_{\underline{\mu} \eta^{\sigma}}$.

To conclude the proof, we need to construct an inverse of $\Psi$. Take a basis monomial $\mathfrak{m}=x_{1}^{i_{1}} \cdots x_{1}^{i_{n}} \in \operatorname{Hom}_{R}\left(\mathrm{C}_{w_{k} z}, \mathrm{C}_{w_{k} z^{\prime}}\right)$ that does not lie in $\widetilde{\mathbf{W}}_{z, z^{\prime}}$. For every $j$, let $\ell_{j}$ be the maximum such that $x_{\vee_{j}^{\mu}} x_{\vee_{j}^{\mu}+1} \cdots x_{\ell_{j}-1}$ divide $\mathfrak{m}$. As $\mathfrak{m}$ does not lie in $\widetilde{W}_{z, z^{\prime}}$, it should be $\ell_{j}<\vee_{j+1}^{\lambda}$ and $\ell_{j}<\vee_{i+1}^{\mu}$. Form a weight $\eta$ in the same block of $\lambda$ and $\mu$ with the $V^{\prime}$ 's in positions $\ell_{1}, \ldots, \ell_{n-k}$. By Lemma 5.5] the diagram $\underline{\mu} \eta \bar{\lambda}$ is oriented. Let $\mathfrak{m}^{\prime}$ be the quotient of $\mathfrak{m}$ by $p_{\mu} \eta^{e}$. By construction, $b_{j}^{\mu}=b_{j}^{\eta}$ if $x_{j}$ does not appear in $p_{\underline{\mu}} \eta^{e}$, and $b_{j}^{\mu}=b_{j}^{\eta}+1$ if $x_{j}$ appears (with coefficient 1) in 
$p_{\underline{\mu} \eta^{e}}$. Hence, it is clear that $\mathfrak{m}^{\prime}$ is a monomial $\mathfrak{S}_{\sigma}^{\prime}\left(x_{\wedge_{1}^{\eta}}, \ldots, x_{\wedge_{k}^{\eta}}\right)$. By construction, it follows that in this way we get an inverse of the map (5.12), which hence is an isomorphism.

As a consequence we obtain the following result, which completes the proof of Theorem 4.17

Lemma 5.9. For all $z, z^{\prime} \in D$ we have

$$
\operatorname{dim}_{\mathbb{C}} \operatorname{Hom}_{R}\left(C_{w_{k} z}, C_{w_{k} z^{\prime}}\right) / \widetilde{W}_{z, z^{\prime}}=\operatorname{dim}_{\mathbb{C}} Z_{z, z^{\prime}} .
$$

Proof. By Proposition 5.8 the dimension of $\operatorname{Hom}_{R}\left(\mathrm{C}_{w_{k} z}, \mathrm{C}_{w_{k} z^{\prime}}\right) / \widetilde{\mathrm{W}}_{z, z^{\prime}}$ is the same as $\operatorname{dim}_{\mathbb{C}} \mathcal{Z}_{\mu, \lambda}$, where $\lambda, \mu \in \Gamma$ are the weights corresponding to $z, z^{\prime}$. This dimension is simply $k$ ! times the number of unenhanced weights $\eta$ such that $\underline{\mu} \eta \bar{\lambda}$ is oriented. By Lemma 6.6 this is the same as $\operatorname{dim} Z_{z, z^{\prime}}$.

Being $\Gamma$ and $D_{n, k}$ identified, we will often write $C_{\lambda}$ for $C_{w_{k} z}$, where $z \in D_{n, k}$ is the element corresponding to $\lambda$. If $a=\underline{\lambda}$ and $b=\bar{\lambda}$ we will even write $C_{a}$ or $C_{b}$ instead of $C_{\lambda}$. We will do similarly for $\mathrm{W}_{z, z^{\prime}}$ and $\mathrm{Z}_{z, z^{\prime}}$.

The algebra structure. Thanks to Proposition 5.8, we can define a graded algebra $A=A_{\Gamma}$ over $\mathbb{C}$. As a graded vector space, a homogeneous basis is given by

$$
\left\{\left(\underline{\alpha} \lambda^{\sigma} \bar{\beta}\right) \mid \text { for all } \alpha, \lambda, \beta \in \Gamma, \sigma \in \mathbb{S}_{k} \text { such that } \alpha \supset \lambda \subset \beta\right\},
$$

which is the same as

$$
\left\{\left(a \lambda^{\sigma} b\right) \mid \text { for all oriented fork diagrams } a \lambda b \text { with } \lambda \in \Gamma\right\} .
$$

The degree on this basis is given by the degrees on fork diagrams. For $\lambda \in \Gamma$ we write $e_{\lambda}$ for $(\underline{\lambda} \lambda \bar{\lambda})$. Note that the vectors $e_{\lambda}$ give a basis for the degree 0 component of $A$.

Example 5.10. Let us consider a block $\Gamma$ of weights with $2 \wedge$ 's and $1 \vee$, that is,

$$
\Gamma=\left\{\lambda_{1}=\wedge \wedge \vee, \lambda_{2}=\wedge \vee \wedge, \lambda_{3}=\vee \wedge \wedge\right\}
$$

Then the basis $\left\{e_{\lambda_{i}}\right\}$ of the degree 0 component is given by

$$
e_{\lambda_{1}}=\uparrow \uparrow \psi, \quad e_{\lambda_{2}}=\uparrow \psi \lambda, \quad e_{\lambda_{3}}=\psi \lambda \lambda
$$

From Proposition 5.8 we get the following:

Corollary 5.11. There is an isomorphism of graded vector spaces

$$
A \cong \bigoplus_{z, z^{\prime} \in D} \operatorname{Hom}\left(\mathrm{C}_{w_{k} z}, \mathrm{C}_{w_{k} z^{\prime}}\right) / \mathrm{W}_{z, z^{\prime}}
$$

This defines a graded algebra structure on $A$.

The product of two basis vectors of $A$ can be computed explicitly using the isomorphism (5.18) as explained in detail in Remark 5.12, Unfortunately, we are not able to describe the multiplication in the algebra $A$ purely in terms of diagrams. Nevertheless, the diagrammatic description proves useful to find other properties of the algebra $A$, as we will explain in the following. 
Remark 5.12. Explicitly, the multiplication of the basis vectors $\left(a \lambda^{\sigma} b\right)$ and $\left(c \mu^{\sigma^{\prime}} d\right)$ can be computed in the following way. First, if $b^{*} \neq c$, then set it to be zero. Now suppose $b=c^{*}$. Then take $p_{c \mu^{\sigma^{\prime}}}$ and $p_{a \lambda^{\sigma}}$ in $R$ and multiply them. By construction, the result gives a well-defined morphism of the corresponding Soergel modules: write it as a linear combination of the basis (4.14) and translate it in the diagrammatic algebra $A$ using the isomorphism of Proposition 5.8 .

Example 5.13. Let
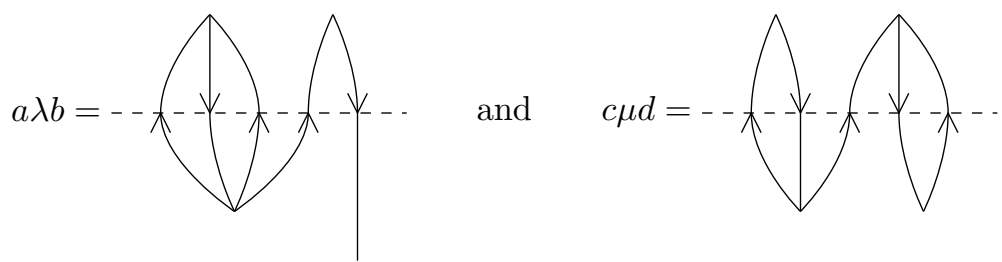

Let also $\sigma=s_{1} \in \mathbb{S}_{3}, \tau=e \in \mathbb{S}_{3}$. We want to compute the product $\left(a \lambda^{\sigma} b\right)\left(c \mu^{\tau} d\right)$. First notice that $b^{*}=c$ (otherwise the product would be trivially zero). By (5.9) we have

$$
\begin{aligned}
p_{a \lambda^{\sigma}} & =x_{1} \cdot x_{1} x_{4}, \\
p_{c \mu^{\tau} d} & =1 \cdot x_{1}
\end{aligned}
$$

(for the computation of the polynomials $\mathfrak{S}_{\sigma}^{\prime}$ and $\mathfrak{S}_{\tau}^{\prime}$ we refer to Example 2.13). The product is $p_{a \lambda^{\sigma}} p_{c \mu^{\tau}}=x_{1}^{3} x_{4}$. The $\boldsymbol{b}$-sequence of $a$ is $(4,3,2,1,1)$; hence $x_{1}^{3} x_{4}$ is not an element of the monomial basis (4.14) of $R_{a}$. We need to do some computations in the ring $R_{a}$ : using the relations $x_{1}+x_{2}+x_{3}+x_{4} \equiv 0$ and $x_{1}^{4} \equiv 0$ we have

$$
x_{1}^{3} x_{4} \equiv-x_{1}^{4}-x_{1}^{3} x_{2}-x_{1}^{3} x_{3} \equiv-x_{1}^{3} x_{2}-x_{1}^{3} x_{3} .
$$

This is now a linear combination of monomials of the basis (4.14). The monomial $-x_{1}^{3} x_{2}$, although not zero in $R_{a}$, is of type (4.27), hence defines an illicit morphism and is zero in the quotient. We are left only with the monomial $\mathfrak{m}=x_{1}^{3} x_{3}$. This is an element of (4.14) and, according to Theorem 4.17, does not define an illicit morphism. We need to translate it into a diagram via Proposition 5.8, The $\wedge \vee-$ sequence corresponding to $a$ is $\vee \wedge \wedge \wedge \vee$; in particular, the indices of the $\vee$ 's are 1,5 . Now, $x_{5}$ does not divide $\mathfrak{m}$, and the biggest index $i$ such that $x_{1} x_{2} \cdots x_{i} \mid \mathfrak{m}$ is 1 . Hence the monomial $\mathfrak{m}$ corresponds to a diagram $a \eta^{\pi} d$ where $\eta$ has $\vee$ 's in positions 2,5 . Moreover, the permutation $\pi$ is determined by $\mathfrak{S}_{\pi}^{\prime}\left(x_{1}, x_{3}, x_{4}\right)=x_{1}^{2} x_{3}$. By Example 2.13, $\pi$ is the longest element of $\mathbb{S}_{3}$. Hence $\left(a \lambda^{\sigma} b\right)\left(c \mu^{\tau} d\right)=-\left(a \eta^{\pi} d\right)$, where

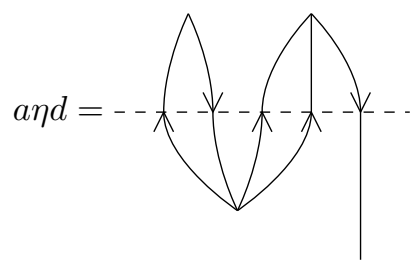

By construction, $p_{\underline{\lambda} \lambda^{e}}=1$ for any $\lambda \in \Gamma$. Under the isomorphism of Proposition 5.8, the element $e_{\lambda}$ is sent to $\operatorname{id}_{\mathrm{C}_{w_{k} z}} \in \operatorname{End}_{R}\left(\mathrm{C}_{w_{k} z}\right)$, where $z \in D$ corresponds to $\lambda$; hence the elements $e_{\lambda}$ satisfy

$$
e_{\lambda}\left(a \mu^{\sigma} b\right)=\left\{\begin{array}{ll}
a \mu^{\sigma} b & \text { if } a=\bar{\lambda}, \\
0 & \text { otherwise, }
\end{array} \quad\left(a \mu^{\sigma} b\right) e_{\lambda}= \begin{cases}a \mu^{\sigma} b & \text { if } b=\underline{\lambda} \\
0 & \text { otherwise }\end{cases}\right.
$$


for any basis element $a \mu^{\sigma} b \in A$. That is, the vectors $\left\{e_{\lambda} \mid \lambda \in \Gamma\right\}$ are mutually orthogonal idempotents whose sum is the identity $1 \in A$. The decomposition (5.18) can be written as

$$
A=\bigoplus_{\lambda, \mu \in \Gamma} e_{\lambda} A e_{\mu}
$$

A basis of the summand $e_{\lambda} A e_{\mu}$ is

$$
\left\{\underline{\lambda} \eta^{\sigma} \bar{\mu} \mid \text { for all } \eta \in \Gamma, \sigma \in \mathbb{S}_{k} \text { such that } \lambda \supset \eta \subset \mu\right\} .
$$

Duality. Recall from $\$ 2.4$ that for every $z, z^{\prime} \in D$ we have an isomorphism

$$
\begin{aligned}
\Theta: \operatorname{Hom}_{R}\left(\mathrm{C}_{w_{k} z}, \mathrm{C}_{w_{k} z^{\prime}}\right) & \longrightarrow \operatorname{Hom}_{R}\left(\mathrm{C}_{w_{k} z^{\prime}}, \mathrm{C}_{w_{k} z}\right), \\
(1 \mapsto p) & \longmapsto\left(1 \mapsto \boldsymbol{x}^{\boldsymbol{b}-\boldsymbol{b}^{\prime}} p\right),
\end{aligned}
$$

where $\boldsymbol{b}$ and $\boldsymbol{b}^{\prime}$ are the $\boldsymbol{b}$-sequences of $z$ and $z^{\prime}$, respectively, $\boldsymbol{b}-\boldsymbol{b}^{\prime}=\left(b_{1}-b_{1}^{\prime}, \ldots\right.$, $\left.b_{n}-b_{n}^{\prime}\right)$ and the notation is as in (2.19).

Lemma 5.14. Let $\lambda, \mu \in \Gamma$ and let $z, z^{\prime}$ be the corresponding elements of $D_{n, k}$. We have $\Theta\left(\mathrm{W}_{z, z^{\prime}}\right)=\mathrm{W}_{z^{\prime}, z}$. Therefore the isomorphism $\Theta$ descends to an isomorphism $\Theta: Z_{z, z^{\prime}} \rightarrow Z_{z^{\prime}, z}$ that fits with the duality on diagrams:

$$
\Theta\left(\Psi\left(\underline{\mu} \eta^{\sigma} \bar{\lambda}\right)\right)=\Psi\left(\underline{\lambda} \eta^{\sigma} \bar{\mu}\right)
$$

for every enhanced weight $\eta^{\sigma}$ such that $\underline{\mu} \eta^{\sigma} \bar{\lambda}$ is oriented.

Proof. Let $\boldsymbol{b}, \boldsymbol{b}^{\prime}$ be the $\boldsymbol{b}$-sequences of $\lambda$ and $\mu$, respectively. Note that

$$
\frac{\boldsymbol{x}^{\boldsymbol{b}-\boldsymbol{1}}}{\boldsymbol{x}^{\boldsymbol{b}^{\prime}-1}}=\boldsymbol{x}^{\boldsymbol{b}-\boldsymbol{b}^{\prime}}=\prod_{\vee_{j}^{\lambda}<\vee_{j}^{\mu}}\left(x_{\vee_{j}^{\lambda}} \cdots x_{\vee_{j}^{\mu}-1}\right) \prod_{\vee_{j}^{\mu}<\vee_{j}^{\lambda}}\left(x_{\vee_{j}^{\mu}}^{-1} \cdots x_{\vee_{j}^{\lambda}-1}^{-1}\right)
$$

as an element in $\mathbb{C}\left[x_{1}^{ \pm 1}, \ldots, x_{n}^{ \pm 1}\right]$. If $(1 \mapsto \mathfrak{m})$ is a monomial morphism of the basis (4.14) of $\operatorname{Hom}_{R}\left(\mathrm{C}_{w_{k} z^{\prime}}, \mathrm{C}_{w_{k} z}\right)$, it follows immediately that $(1 \mapsto \mathfrak{m}) \in \mathrm{W}_{z^{\prime}, z}$ if and

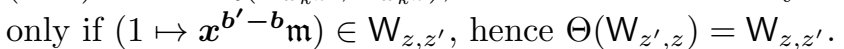

Moreover, it follows from equation (5.9) for the polynomials $p_{\underline{\lambda} \eta^{\sigma}}$ and $p_{\mu} \eta^{\sigma}$ that $p_{\underline{\mu} \eta^{\sigma}}=x^{\boldsymbol{b}-\boldsymbol{b}^{\prime}} p_{\underline{\lambda} \eta^{\sigma}}$, hence $\Theta\left(\Psi\left(\underline{\mu} \eta^{\sigma} \bar{\lambda}\right)\right)=\Psi\left(\underline{\lambda} \eta^{\sigma} \bar{\mu}\right)$.

As a corollary, we have that the linear map $\star: A \rightarrow A$ defined by

$$
(a \lambda b)^{\star}=\left(b^{*} \lambda a^{*}\right)
$$

is an algebra anti-isomorphism.

As follows from the definition, the algebra $A$ depends only on the number of $\wedge$ 's and $\vee$ 's in the block $\Gamma$.

Definition 5.15. We define $A_{n, k}=A_{\Gamma}$ for some block $\Gamma$ with $k \wedge$ 's and $n-k \vee$ 's.

5.3. Graded cellular structure. We construct here explicitly the graded cellular structure of the algebra $A$. The construction follows the one in BS11a]. 
Proposition 5.16. Let $(a \lambda b)$ and $(c \mu d)$ be basis vectors of $A$. Then the product $\left(a \lambda^{\sigma} b\right)\left(c \mu^{\tau} d\right)$ is equal to:

$$
\begin{cases}0 & \text { if } b \neq c^{*}, \\
\left(a \mu^{\tau} d\right) & \begin{array}{l}
\text { if } b=c^{*}=\bar{\lambda}, \sigma=e \text { and } \\
(a \mu d) \text { is oriented, }
\end{array} \\
\sum_{\ell\left(\tau^{\prime}\right)>\ell(\tau)} t_{\left(a \lambda^{\sigma} c\right)}^{\tau^{\prime}}\left(\mu^{\tau}\right) \cdot\left(a \mu^{\tau^{\prime}} d\right)+(\dagger) & \begin{array}{l}
\text { if } b=c^{*},(a \mu d) \text { is oriented, } \\
\text { and either } b \neq \bar{\lambda} \text { or } \sigma \neq e, \\
(\dagger)
\end{array} \\
\text { otherwise, }\end{cases}
$$

where:

(i) the scalars $t_{\left(a \lambda^{\sigma} c\right)}^{\tau^{\prime}}\left(\mu^{\tau}\right)$ are independent of $d$;

(ii) $(\dagger)$ denotes a linear combination of basis vectors of $A$ of the form $\left(a \nu^{\chi} d\right)$ with $\nu \succ \mu$.

Proof. If $b \neq c^{*}$ the claim is obvious, so let us suppose $b=c^{*}$. Suppose moreover that there is some weight $\nu$ such that $a \nu d$ is oriented (or equivalently that $Z_{d, a}$ is not trivial); otherwise the claim is also obvious.

Of course we have

$$
\left(a \lambda^{\sigma} b\right)\left(c \mu^{\tau} d\right)=\sum_{\nu \in \Gamma, \chi \in \mathbb{S}_{k}} C\left(\nu^{\chi}\right)\left(a \nu^{\chi} d\right)
$$

for some coefficients $C\left(\nu^{\chi}\right) \in \mathbb{C}$. Let us first prove that only terms with $\nu^{\chi} \succeq \mu^{\tau}$ occur in the sum; i.e., if $C\left(\nu^{\chi}\right) \neq 0$, then $\nu^{\chi} \succeq \mu^{\tau}$.

Before continuing, let us stress the subtlety in the argument. We want to understand which element of $A$ corresponds to the morphism $1 \mapsto p_{a \lambda^{\sigma}} p_{c \mu^{\tau}}$ : in general this morphism is not a monomial morphism of the basis (4.14), and we have to use the relations defining $R_{a}$ to rewrite it as a linear combination of the monomial morphisms (4.14).

Let us fix some $\nu^{\chi}$ such that $C\left(\nu^{\chi}\right) \neq 0$. First, let us prove that $\nu \succeq \mu$. By definition, $\nu \succeq \mu$ is equivalent to $\vee_{j}^{\nu} \geq \vee_{j}^{\mu}$ for all $j=1, \ldots, n-k$. Fix an index $j$. If $\vee_{j}^{a} \geq \vee_{j}^{\mu}$, then also $\vee_{j}^{\nu} \geq \vee_{j}^{\mu}$ by Lemma 5.5. (i). Hence suppose $\vee_{j}^{a}<\vee_{j}^{\mu}$. By construction, the monomial

$$
\left(x_{\vee_{j}^{a}} x_{\vee_{j}^{a}+1} \cdots x_{\vee_{j}^{\lambda}-1}\right)\left(x_{\vee_{j}^{c}} x_{\bigvee_{j}^{c}+1} \cdots x_{\bigvee_{j}^{\mu}-1}\right)
$$

divides $p_{a \lambda^{\sigma}} p_{c \mu^{\tau}}$. In particular, since $\vee_{j}^{\lambda} \geq \vee_{j}^{b}=\vee_{j}^{c}$, also $x_{\vee_{j}^{a}} x_{\vee_{j}^{a}+1} \cdots x_{\vee_{j}^{\mu}-1}$ divides $p_{a \lambda^{\sigma}} p_{c \mu^{\tau}}$. Hence, if $p_{a \lambda^{\sigma}} p_{c \mu^{\tau}}$ is a monomial of the basis (4.14), we can conclude that $\vee_{j}^{\nu} \geq \vee_{j}^{\mu}$. Otherwise, we get the same conclusion using the technical Lemma 5.17 below.

Now to check that $\nu^{\chi} \succeq \mu^{\tau}$ we have to show that in the case $\nu=\mu$ we have $\ell(\chi) \geq \ell(\tau)$. So let us suppose $\nu=\mu$. Since the multiplication is graded, we must have

$$
\operatorname{deg}\left(a \lambda^{\sigma} b\right)+\operatorname{deg}\left(c \mu^{\tau} d\right)=\operatorname{deg}\left(a \mu^{\chi} d\right) .
$$

If $a=\rho$ we write $\ell(a)$ for $\ell(\rho)$, and similarly for $b, c, d$. Then, using Lemma 5.7, we get from (5.32)

$$
2 \ell(\chi)=2 \ell(\tau)+2 \ell(\sigma)+2 \ell(b)-2 \ell(\lambda) .
$$


Since $\lambda^{\sigma} b$ is oriented, by Lemma 5.4 the diagram $b$ corresponds to some weight that is smaller than or equal to $\lambda$ in the Bruhat order. This implies that $\ell(\lambda) \leq \ell(b)$ (notice that under the identification of $\Gamma$ with $D_{n, k}$, the Bruhat order on weights corresponds to the opposite of the usual Bruhat order on permutations). It follows that $\ell(\chi) \geq \ell(\tau)$. Hence we have shown that

$$
\left(a \lambda^{\sigma} b\right)\left(c \mu^{\tau} d\right)=\sum_{\ell\left(\tau^{\prime}\right) \geq \ell(\tau)} C\left(\mu^{\tau^{\prime}}\right)\left(a \mu^{\tau^{\prime}} d\right)+\sum_{\nu \succ \mu, \chi \in \mathbb{S}_{k}} C\left(\nu^{\chi}\right)\left(a \nu^{\chi} d\right) .
$$

Now suppose that $C\left(\mu^{\xi}\right) \neq 0$ for some $\xi \in \mathbb{S}_{k}$ with $\ell(\xi)=\ell(\tau)$. If we substitute in (5.33) $\chi=\xi$, we get $2 \ell(\sigma)+2 \ell(b)-2 \ell(\lambda)=0$. Since $\ell(b) \geq \ell(\lambda)$, we must have $\ell(\sigma)=0$ and $\ell(b)=\ell(\lambda)$. This implies $\sigma=e$ and $b=\bar{\lambda}$. It is easy to see that in this case the morphism $1 \mapsto p_{a \lambda^{\sigma}} p_{c \mu^{\tau}}$ is an element of the monomial basis (4.14), and hence we have exactly $\left(a \lambda^{\sigma} b\right)\left(c \mu^{\tau} d\right)=\left(a \mu^{\tau} d\right)$. This shows the second case of (5.29) and also that if either $b \neq \bar{\lambda}$ or $\sigma \neq e$, then we can rewrite (5.34) as

$$
\left(a \lambda^{\sigma} b\right)\left(c \mu^{\tau} d\right)=\sum_{\ell\left(\tau^{\prime}\right)>\ell(\tau)} C\left(\mu^{\tau^{\prime}}\right)\left(a \mu^{\tau^{\prime}} d\right)+\sum_{\nu \succ \mu, \chi \in \mathbb{S}_{k}} C\left(\nu^{\chi}\right)\left(a \nu^{\chi} d\right) .
$$

Since $C\left(\mu^{\tau^{\prime}}\right)$ is automatically zero unless $a \mu d$ is oriented, this concludes the proof of (5.29) and (ii).

We are left to show (i), In order to determine the coefficients of (5.30), consider the expression of the polynomial $p_{a \lambda^{\sigma}} p_{c \mu^{\tau}}$ in the basis (4.13) of $R_{a}$ :

$$
p_{a \lambda \sigma} p_{c \mu^{\tau}}=\sum_{\boldsymbol{j} \in J} \alpha_{\boldsymbol{j}} \boldsymbol{x}^{\boldsymbol{j}}
$$

Define $J^{\prime \prime} \subseteq J$ to be the subset of tuples $\boldsymbol{j}$ such that the morphism $\left(1 \mapsto \boldsymbol{x}^{\boldsymbol{j}}\right) \in$ $\operatorname{Hom}_{R}\left(\mathrm{C}_{d}, \mathrm{C}_{a}\right)$ dies in the quotient $\mathrm{Z}_{d, a}$, since it is divided by some morphism of the type (ii) of Theorem 4.17. Let also $J^{\prime}=J \backslash J^{\prime \prime}$. Fix some $j \in J^{\prime}$. By Proposition 5.8, the basis morphism $\left(1 \mapsto \boldsymbol{x}^{\boldsymbol{j}}\right) \in \mathrm{Z}_{d, a}$ corresponds to a diagram $a \nu^{\chi} d$; then we have $C\left(\nu^{\chi}\right)=\alpha_{j}$. Notice that the unique dependence on $d$ is in determining the subset $J^{\prime \prime} \subseteq J$.

Now suppose $a \mu d$ is oriented, fix some $\tau^{\prime} \in \mathbb{S}_{k}$ and let $\left(1 \mapsto \boldsymbol{x}^{j}\right) \in \mathrm{Z}_{d, a}$ be the morphism of the basis Lemma 4.14 corresponding to the diagram $a \mu^{\tau^{\prime}} d$. By the definition of orientation, for all $i$ we have $\vee_{i}^{d} \leq \vee_{i}^{\mu}<\vee_{i+1}^{d}$ and $\vee_{i}^{a} \leq \vee_{i}^{\mu}<\vee_{i+1}^{a}$. Since $\boldsymbol{x}^{j} \in \mathbb{C}\left[x_{\wedge_{1}^{\mu}}, \ldots, x_{\wedge_{k}^{\mu}}\right]$, neither $x_{\vee_{i}^{d}} x_{\vee_{i}^{d}+1} \cdots x_{\vee_{i+1}^{d}}$ nor $x_{\vee_{i}^{d}} x_{\vee_{i}^{d}+1} \cdots x_{\vee_{i+1}^{a}}$ can divide $\boldsymbol{x}^{j}$. Hence for all $d$ such that $a \mu d$ is oriented we have $\left(1 \mapsto \boldsymbol{x}^{\boldsymbol{j}}\right) \notin \mathrm{W}_{d, a}$ and with the notation of the preceding paragraph $j \in J^{\prime}$. Hence $C\left(\mu^{\tau^{\prime}}\right)$ is independent of $d$, proving (i).

Lemma 5.17. Fix some $\boldsymbol{b} \in \mathscr{B}$ and let $m$ be an index such that $b_{m-1}=b_{m}$. Suppose that $x_{m} x_{m+1} \cdots x_{m+\ell}$ divides some polynomial $p \in R$. Write $p=\sum_{\boldsymbol{i}} c_{\boldsymbol{i}} \boldsymbol{x}^{\boldsymbol{i}}$ in $R_{\boldsymbol{b}}$, where $\boldsymbol{x}^{\boldsymbol{i}}$ are monomials of the basis (4.14). Then $x_{m} x_{m+1} \cdots x_{m+\ell}$ divides all monomials $x^{\boldsymbol{i}}$ for which $c_{\boldsymbol{i}} \neq 0$.

Proof. We will use the relations defining the ideal $I_{\boldsymbol{b}}$ to write the expression of $p$ as a linear combination of basis monomials. Of course, it is sufficient to examine the case in which $p=\boldsymbol{x}^{\boldsymbol{j}}$ is a monomial.

Consider the maximum $r$ for which $j_{r} \geq b_{r}$ : if there is no such $r$, then $p$ is a monomial of the basis (4.14) and we are done. If $r<m$ or $r>m+\ell$, then using the relation $h_{b_{r}}\left(x_{1}, \ldots, x_{r}\right)$ we can rewrite $p$ as a linear combination of monomials 
$\boldsymbol{x}^{\boldsymbol{j}^{\prime}}$ with $j_{r}^{\prime}<j_{r}$ and $x_{m} x_{m+1} \cdots x_{m+\ell} \mid \boldsymbol{x}^{\boldsymbol{j}^{\prime}}$, so by an induction argument we may suppose $m<r<m+\ell$. If $\ell \geq 1$ we can write

$$
x_{r-1} x_{r}^{j_{r}}=x_{r-1} h_{j_{r}}\left(x_{1}, \ldots, x_{r}\right)-\sum_{s=0}^{j_{\ell}-1} x_{\ell-1} x_{r}^{s} h_{j_{r}-s}\left(x_{1}, \ldots, x_{r-1}\right) .
$$

Since $h_{j_{r}}\left(x_{1}, \ldots, x_{r}\right) \in I_{\boldsymbol{b}}$ because $j_{r} \geq b_{r}$, and also $x_{r-1} h_{j_{r}}\left(x_{1}, \ldots, x_{r-1}\right) \in I_{\boldsymbol{b}}$ by (2.3), the expression (5.37) gives in $R_{\boldsymbol{b}}$

$$
x_{r-1} x_{r}^{j_{r}} \equiv \sum_{s=1}^{j_{r}-1} x_{r-1} x_{r}^{s} h_{j_{r}-s}\left(x_{1}, \ldots, x_{r-1}\right) \bmod I_{\boldsymbol{b}} .
$$

In the special case $\ell=0, r=m$, we write instead

$$
x_{m}^{j_{m}}=h_{j_{m}}\left(x_{1}, \ldots, x_{m}\right)-\sum_{s=0}^{j_{m}-1} x_{m}^{s} h_{j_{m}-s}\left(x_{1}, \ldots, x_{m-1}\right),
$$

which in $R_{b}$ is

$$
x_{m}^{j_{m}} \equiv-\sum_{s=1}^{j_{m}-1} x_{m}^{s} h_{j_{m}-s}\left(x_{1}, \ldots, x_{m-1}\right) \quad \bmod I_{\boldsymbol{b}},
$$

since $j_{m} \geq b_{m-1}, b_{m}$. Both in (5.38) and (5.40), on the r.h.s. we have a sum of monomials $\boldsymbol{x}^{j^{\prime}}$ with $1 \leq j_{r}^{\prime}<j_{r}$ : by an induction argument on $j_{r}$, the claim follows.

The main result of this subsection is the graded cellular algebra structure of $A$ in the sense of [GL96], HM10. A graded cellular algebra is an associative unital algebra $H$ together with a cell datum $(X, I, C$, deg) such that:

(GC1) $X$ is a finite partially ordered set;

(GC2) $I(\lambda)$ is a finite set for each $\lambda \in X$;

(GC3) $C: \dot{U}_{\lambda \in X} I(\lambda) \times I(\lambda) \rightarrow H,(i, j) \mapsto C_{i, j}^{\lambda}$ is an injective map whose image is a basis of $H$;

(GC4) the map $H \rightarrow H, C_{i, j}^{\lambda} \mapsto C_{j, i}^{\lambda}$ is an algebra anti-automorphism;

(GC5) if $\lambda \in X$ and $i, j \in I(\lambda)$, then for any $x \in H$ we have that

$$
x C_{i, j}^{\lambda} \equiv \sum_{i^{\prime} \in I(\lambda)} r_{x}\left(i^{\prime}, i\right) C_{i^{\prime}, j}^{\lambda} \quad\left(\bmod H_{>\lambda}\right),
$$

where the scalar $r_{x}\left(i^{\prime}, i\right)$ is independent of $j$ and $H_{>\lambda}$ is the subspace of $H$ spanned by $\left\{C_{h, l}^{\mu} \mid \mu>\lambda\right.$ and $\left.k, l \in I(\mu)\right\}$;

(GC6) deg : $\dot{\bigcup}_{\lambda \in X} I(\lambda) \rightarrow \mathbb{Z}, i \mapsto \operatorname{deg}_{i}^{\lambda}$ is a function such that the $\mathbb{Z}$-grading on $H$ defined by declaring $\operatorname{deg} C_{i, j}^{\lambda}=\operatorname{deg}_{i}^{\lambda}+\operatorname{deg}_{j}^{\lambda}$ makes $H$ into a graded algebra.

We have:

Proposition 5.18. The algebra $A$ is a graded cellular algebra with cell datum $\left(\left(\Gamma \times \mathbb{S}_{k}, \preceq\right), I, C, \operatorname{deg}\right)$ where:

(a) $I\left(\lambda^{\sigma}\right)=\{\alpha \in \Gamma \mid \alpha \subset \lambda\}$;

(b) $C$ is defined by setting $C_{\alpha, \beta}^{\lambda^{\sigma}}=\left(\underline{\alpha} \lambda^{\sigma} \bar{\beta}\right)$;

(c) $\operatorname{deg}_{\alpha}^{\lambda^{\sigma}}=\operatorname{deg}\left(\underline{\alpha} \lambda^{\sigma}\right)-\ell(\sigma)$. 
Proof. Conditions (GQ1] 3) and (GC6) are direct consequences of the definitions. Condition (GC4) follows from Lemma 5.14, Condition (GC5) follows from Proposition 5.16 .

5.4. Properly stratified structure. As before, let us fix a block $\Gamma$ and let $A=A_{\Gamma}$. We construct now explicitly a properly stratified structure on $A$. The construction is similar to the one of BS11a.

An $A$-module will always be a finite dimensional graded left $A$-module. Let $A$-gmod be the category of such modules. If $M=\bigoplus M_{i}$ is a graded $A$-module, then we will write $M\langle j\rangle$ for the same module structure but with new grading defined by $(M\langle j\rangle)_{i}=M_{i-j}$. If $M, N$ are graded $A$-modules, then $\operatorname{Hom}_{A}(M, N)$ is a graded vector space.

Irreducible and projective $A$-modules. As we already noticed, the algebra $A$ is unital with $1=\sum_{\lambda \in \Gamma} e_{\lambda}$. Let $A_{>0}$ be the sum of all components of $A$ of strictly positive degree. Then

$$
A / A_{>0}=\bigoplus_{\lambda} e_{\lambda} \mathbb{C} e_{\lambda} \cong \bigoplus_{\lambda \in \Gamma} \mathbb{C}
$$

is a split semisimple algebra, with a basis given by the images of the idempotents $e_{\lambda}$. The image of $e_{\lambda}$ spans a one dimensional $A / A_{>0}$-modules, and hence also a one dimensional $A$-module which we denote $L(\lambda)$. Thus $L(\lambda)$ is a copy of the field concentrated in degree 0 , and $\left(a \mu^{\sigma} b\right) \in A$ acts on it as 1 if $\left(a \mu^{\sigma} b\right)=\left(\underline{\lambda} \lambda^{e} \bar{\lambda}\right)$ and as 0 otherwise. The modules

$$
\{L(\lambda)\langle j\rangle \mid \lambda \in \Gamma, j \in \mathbb{Z}\}
$$

give a complete set of isomorphism classes of irreducible graded $A$-modules.

For any finite dimensional graded $A$-module $M$, let $M^{*}$ denote its graded dual. That is, $\left(M^{*}\right)_{j}=\operatorname{Hom}_{\mathbb{C}}\left(M_{-j}, \mathbb{C}\right)$ and $x \in A$ acts on $f \in M^{*}$ by $x f(m)=f\left(x^{\star} m\right)$. As $e_{\lambda}^{\star}=e_{\lambda}$ we have that

$$
L(\lambda)^{*} \cong L(\lambda)
$$

for each $\lambda \in \Gamma$.

For each $\lambda \in \Gamma$ also let $P(\lambda)=A e_{\lambda}$. This is a graded $A$-module with basis

$$
\left\{\left(\underline{\nu} \mu^{\sigma} \bar{\lambda}\right) \mid \text { for all } \nu, \mu \in \Gamma \text { and } \sigma \in \mathbb{S}_{k} \text { with } \nu \subset \mu \supset \lambda\right\} \text {. }
$$

The module $P(\lambda)$ is a projective module; in fact, it is the projective cover of $L(\lambda)$ in $A$-gmod. The modules

$$
\{P(\lambda)\langle j\rangle \mid \lambda \in \Gamma, j \in \mathbb{Z}\}
$$

give a complete set of isomorphism classes of indecomposable projective $A$-modules.

Cell modules and standard modules. We introduce now standard modules. The terminology will be motivated at the end of the section. For $\mu \in \Gamma$, define $\Delta(\mu)$ to be the vector space with basis

$$
\left\{\left(\underline{\lambda} \mu^{\tau}|| \text { for all } \lambda \in \Gamma, \tau \in \mathbb{S}_{k} \text { such that } \lambda \subset \mu\right\}\right.
$$

or, equivalently,

$$
\left\{\left(c \mu^{\tau}|| \text { for all oriented lower fork diagrams } c \mu^{\tau}\right\} .\right.
$$


We put a grading on $\Delta(\mu)$ by defining the degree of $\left(c \mu^{\tau} \mid\right.$ to be $\operatorname{deg}\left(c \mu^{\tau}\right)$, and we make it into an $A$-module through

$$
\left(a \lambda^{\sigma} b\right)\left(c \mu^{\tau} \mid= \begin{cases}\sum_{\tau^{\prime} \in \mathbb{S}_{k}} t_{\left(a \lambda^{\sigma} b\right)}^{\tau^{\prime}}\left(\mu^{\tau}\right)\left(a \mu^{\tau^{\prime}} \mid\right. & \text { if } b=c^{*} \text { and }(a \mu) \text { is oriented } \\ 0 & \text { otherwise }\end{cases}\right.
$$

where $t_{\left(a \lambda^{\sigma} b\right)}^{\tau^{\prime}}\left(\mu^{\tau}\right)$ is the scalar defined by Proposition [5.16. Note that $t_{\left(a \lambda^{\sigma} b\right)}^{\tau^{\prime}}\left(\mu^{\tau}\right)$ was defined only for $\tau^{\prime}=\tau$ or for $\ell\left(\tau^{\prime}\right)>\ell(\tau)$; otherwise we set $t_{\left(a \lambda^{\sigma} b\right)}^{\tau^{\prime}}\left(\mu^{\tau}\right)=0$.

Proposition 5.19. For $\lambda \in \Gamma$ enumerate the distinct elements of the set $\{\mu \in \Gamma \mid \mu \supset \lambda\}$ as $\mu_{1}, \mu_{2}, \ldots, \mu_{m}=\lambda$ so that if $\mu_{i} \prec \mu_{j}$, then $i>j$. Set $M(0)=\{0\}$ and for $i=1, \ldots, m$ define $M(i)$ to be the subspace of $P(\lambda)$ generated by $M(i-1)$ and the vectors

$$
\left\{\left(c \mu_{i}^{\tau} \bar{\lambda}\right) \mid \text { for all oriented lower fork diagrams } c \mu_{i}^{\tau}\right\} .
$$

Then

$$
\{0\}=M(0) \subset M(1) \subset \cdots \subset M(m)=P(\lambda)
$$

is a filtration of $P(\lambda)$ as an $A$-module such that

$$
M(i) / M(i-1) \cong \Delta\left(\mu_{i}\right)\left\langle\operatorname{deg} \mu_{i} \bar{\lambda}\right\rangle
$$

for each $i=1, \ldots, m$.

Proof. It follows from Proposition 5.16 that $M(i)$ is indeed a submodule of $P(\lambda)$. The map

$$
\begin{aligned}
f_{i}: \Delta\left(\mu_{i}\right)\left\langle\operatorname{deg} \mu_{i} \bar{\lambda}\right\rangle & \longrightarrow M(i) / M(i-1) \\
\left(c \mu_{i}^{\tau} \mid\right. & \longmapsto\left(c \mu_{i}^{\tau} \bar{\lambda}\right)+M(i-1)
\end{aligned}
$$

gives an isomorphism of graded vector spaces. This map is of degree zero because

$$
\operatorname{deg}\left(c \mu_{i}^{\tau} \bar{\lambda}\right)=\operatorname{deg}\left(c \mu_{i}^{\tau}\right)+\operatorname{deg}\left(\mu_{i} \bar{\lambda}\right) .
$$

Through this vector space isomorphism we can transport the $A$-module structure of $M(i) / M(i-1)$ to $\Delta\left(\mu_{i}\right)$. Using Proposition 5.16 we see that the module structure we get on $\Delta\left(\mu_{i}\right)$ is given by (5.49). Hence (5.49) defines indeed an $A$-module structure on $\Delta\left(\mu_{i}\right)$ and (5.53) is an isomorphism of $A$-modules. Since any weight $\mu$ arises as $\mu_{i}$ for some $\lambda$ as in the statement of the theorem (take for example $\lambda=\mu$, $i=m$ ), we conclude also that (5.49) defines an $A$-module structure for every $\mu$.

Remark 5.20. In particular, it follows from Proposition 5.19 that each projective module $P(\lambda)$ has a standard filtration, that is, a filtration with standard modules. The function $\operatorname{ht}(\lambda)=\ell(\lambda)$, where we consider $\lambda$ as an element of $D_{n, k}$ via our bijection, plays the role of a height function, as in DM07. All subquotients of the standard filtration (5.51) of $P(\lambda)$ have the form $\Delta(\mu)\langle h\rangle$, where $h \geq 0$ and $\operatorname{ht}(\mu)=\operatorname{ht}(\lambda)-h$, and $h=0$ is possible only if $\mu=\lambda$.

Let us now define cell modules. Let $\mu^{\tau} \in \Gamma \times \mathbb{S}_{k}$ be an enhanced weight and define $V\left(\mu^{\tau}\right)$ to be the vector space with basis

$$
\left\{\left(\underline{\lambda} \mu^{\tau}\right\rfloor \mid \text { for all } \lambda \in \Gamma \text { such that } \lambda \subset \mu\right\}
$$

or, equivalently,

$\left\{\left(c \mu^{\tau}\right\rfloor \mid\right.$ for all oriented lower fork diagrams $\left.c \mu^{\tau}\right\}$. 
Here the symbol 」 has no particular meaning (as also | in (5.47)) and is used only as a symbol to distinguish the basis of cell modules. We remark that the difference from (5.47) and (5.48) is that now the permutation $\tau$ is fixed. As before, we put a grading on $V\left(\mu^{\tau}\right)$ by defining the degree of $\left(c \mu^{\tau}\right\rfloor$ to be $\operatorname{deg}\left(c \mu^{\tau}\right)$, and we make it into an $A$-module through

$$
\left(a \lambda^{\sigma} b\right)\left(c \mu^{\tau}\right\rfloor= \begin{cases}t_{\left(a \lambda^{\sigma} b\right)}^{\tau}\left(\mu^{\tau}\right) \cdot\left(a \mu^{\tau}\right\rfloor & \text { if } b=c^{*} \text { and }(a \mu) \text { is oriented, } \\ 0 & \text { otherwise. }\end{cases}
$$

From Proposition 5.16 we have that $t_{\left(a \lambda^{\sigma} b\right)}^{\tau}\left(\mu^{\tau}\right)$ does not depend on $\tau$. Hence (5.57) is the same as

$$
\left(a \lambda^{\sigma} b\right)\left(c \mu^{\tau}\right\rfloor= \begin{cases}\left(a \mu^{\tau}\right\rfloor & \text { if } b=c^{*}=\bar{\lambda}, \sigma=e \text { and }(a \mu) \text { is oriented, } \\ 0 & \text { otherwise. }\end{cases}
$$

It will follow from Proposition 5.21 that this indeed defines an $A$-module structure. It is clear from (5.58) that all cell modules $V\left(\mu^{\tau}\right)$ for a fixed $\mu$ are isomorphic (up to a degree shift). Explicitly we have $V\left(\mu^{\tau}\right) \cong V\left(\mu^{e}\right)\langle\operatorname{deg}(\tau)\rangle$. We recall that $\operatorname{deg}(\tau)=2 \ell(\tau)$. Therefore for a weight $\mu \in \Gamma$ we define the proper standard module $\bar{\Delta}(\mu)$ to be the vector space with basis

$$
\{(\underline{\lambda} \mu\rfloor \mid \text { for all } \lambda \in \Gamma \text { such that } \lambda \subset \mu\}
$$

or, equivalently,

$$
\{(c \mu\rfloor \mid \text { for all unenhanced oriented lower fork diagrams } c \mu\} \text {. }
$$

We put a grading on $\bar{\Delta}(\mu)$ by defining the degree of $(c \mu\rfloor$ to be $\operatorname{deg}(c \mu)$, and we make it into an $A$-module through

$$
\left(a \lambda^{\sigma} b\right)(c \mu\rfloor= \begin{cases}(a \mu\rfloor & \text { if } b=c^{*}=\bar{\lambda}, \sigma=e \text { and }(a \mu) \text { is oriented, } \\ 0 & \text { otherwise. }\end{cases}
$$

Of course we have an isomorphism $\bar{\Delta}(\mu) \cong V\left(\mu^{e}\right)$.

Proposition 5.21. Let $\mu \in \Gamma$. Enumerate the elements of $\mathbb{S}_{k}$ as $\sigma_{1}, \sigma_{2}, \ldots, \sigma_{k !}=e$ in such a way that if $\ell\left(\sigma_{i}\right)>\ell\left(\sigma_{j}\right)$, then $i<j$. Let $N(0)=\{0\}$ and for $i=1, \ldots, k$ ! define $N(i)$ to be the subspace of $\Delta(\mu)$ generated by $N(i-1)$ and the vectors

$$
\left\{\left(c \mu^{\sigma_{i}}|| \text { for all oriented lower fork diagrams } c \mu^{\sigma_{i}}\right\}\right. \text {. }
$$

Then

$$
\{0\}=N(0) \subset N(1) \subset \cdots \subset N(k !)=\Delta(\mu)
$$

is a filtration of $\Delta(\mu)$ as an $A$-module such that

$$
N(i) / N(i-1) \cong \bar{\Delta}(\mu)\left\langle 2 \ell\left(\sigma_{i}\right)\right\rangle .
$$

Proof. It follows from Proposition 5.16 that $N(i)$ is indeed a submodule of $\Delta(\mu)$. The map

$$
\begin{aligned}
f_{i}: \bar{\Delta}(\mu)\left\langle 2 \ell\left(\sigma_{i}\right)\right\rangle & \longrightarrow N(i) / N(i-1) \\
(c \mu\rfloor & \longmapsto\left(c \mu^{\sigma_{i}} \mid+N(i-1)\right.
\end{aligned}
$$

gives an isomorphism of graded vector spaces. The degree shift comes from

$$
\operatorname{deg}\left(c \mu^{\sigma_{i}}\right)=\operatorname{deg}(c \mu)+2 \ell\left(\sigma_{i}\right) .
$$


Through $f_{i}$ we can transport the module structure of $N(i) / N(i-1)$ to $\bar{\Delta}(\mu)$. The module structure on $N(i) / N(i-1)$ is described by (5.49). It follows that $\bar{\Delta}(\mu)\left\langle 2 \ell\left(\sigma_{i}\right)\right\rangle$ is endowed with the module structure of $V\left(\mu^{\sigma_{i}}\right)$ described by (5.57); this shows in particular that (5.57) defines indeed an $A$-module structure. We have already argued that this is the same as the module structure described by (5.61) on $\bar{\Delta}(\mu)$.

Proposition 5.22. For $\mu \in \Gamma$, let $Q(j)$ be the submodule of $\bar{\Delta}(\mu)$ spanned by all homogeneous vectors of degree $\geq j$. Then

$$
\bar{\Delta}(\mu)=Q(0) \supseteq Q(1) \supseteq Q(2) \supseteq \cdots
$$

is a (finite) filtration of $\bar{\Delta}(\mu)$ as an $A$-module such that

$$
Q(j) / Q(j+1) \cong \bigoplus_{\substack{\lambda \subset \mu \text { with } \\ \operatorname{deg}(\underline{\lambda} \mu)=j}} L(\lambda)\langle j\rangle
$$

for all $j \geq 0$.

Proof. Since $A$ is positively graded, it is clear that each $Q(j)$ is a submodule. The quotient $Q(j) / Q(j+1)$ has basis

$$
\{(\underline{\lambda} \mu\rfloor+Q(j+1) \mid \text { for all } \lambda \in \Gamma \text { such that } \lambda \subset \mu \text { and } \operatorname{deg}(\underline{\lambda} \mu)=j\} \text {. }
$$

We need to show that for each $\lambda$ which occurs the one dimensional subspace $Q^{\prime}(\lambda)$ of $Q(j) / Q(j+1)$ spanned by $(\underline{\lambda} \mu\rfloor+Q(j+1)$ is an $A$-module isomorphic to $L(\lambda)\langle j\rangle$. It is clear where the degree shift comes from. If $x \in A$ has $\operatorname{deg}(x)>0$, then obviously $x$ vanishes on $Q(j) / Q(j+1)$. So let us consider $e_{\nu} \in A$. It follows from (5.61) that

$$
e_{\nu} \cdot(\underline{\lambda} \mu\rfloor= \begin{cases}(\underline{\lambda} \mu\rfloor & \text { if } \nu=\lambda, \\ 0 & \text { otherwise. }\end{cases}
$$

Hence $Q^{\prime}(\lambda)$ is isomorphic to $L(\lambda)$ after the opportune degree shift.

The Grothendieck group. The Grothendieck group $K(A$-gmod) of $A$-gmod is a free $\mathbb{Z}$-module with basis given by equivalence classes of simple modules. The group $K(A$-gmod $)$ becomes a $\mathbb{Z}\left[v, v^{-1}\right]$-module if we set $v[M]=[M\langle 1\rangle]$ for all graded $A$-modules $M$. It is also free as a $\mathbb{Z}\left[v, v^{-1}\right]$-module, with basis $\{[L(\lambda)] \mid \lambda \in \Gamma\}$.

For $\lambda, \mu \in \Gamma$, define

$$
d_{\lambda, \mu}= \begin{cases}v^{\operatorname{deg}(\underline{\lambda} \mu)} & \text { if } \lambda \subset \mu, \\ 0 & \text { otherwise. }\end{cases}
$$

By Propositions 5.19, 5.22 and 5.21 respectively we have that

$$
\begin{aligned}
& {[P(\lambda)]=\sum_{\mu \in \Gamma} d_{\lambda, \mu}[\Delta(\mu)],} \\
& {[\bar{\Delta}(\mu)]=\sum_{\lambda \in \Gamma} d_{\lambda, \mu}[L(\lambda)],} \\
& {[\Delta(\mu)]=[k]_{0} ! \cdot[\bar{\Delta}(\mu)],}
\end{aligned}
$$

where we set

$$
[k]_{0}=\frac{v^{2 k}-1}{v^{2}-1} \quad \text { and } \quad[k]_{0} !=[k]_{0}[k-1]_{0} \cdots[1]_{0} .
$$


Since $d_{\lambda, \lambda}=1$, the matrix $\left(d_{\lambda, \mu}\right)$ is upper triangular with determinant 1 ; hence it is invertible over $\mathbb{Z}\left[v, v^{-1}\right]$. In particular, the proper standard modules give also a $\mathbb{Z}\left[v, v^{-1}\right]$-basis of $[A$-gmod $]$. On the other side, notice that the matrix $[k]_{0}$ ! Id is not invertible over $\mathbb{Z}\left[v, v^{-1}\right]$ unless $k=0,1$. In particular, standard and projective modules do not give a basis of the Grothendieck group in general.

We recall the definition of a graded properly stratified algebra in the sense of [Maz04] (see also [FKM02, Fri07]).

Definition 5.23. Let $B$ be a finite dimensional associative graded algebra over a field $\mathbb{K}$ with a simple preserving duality and with equivalence classes of simple modules $\{\mathbb{L}(\lambda)\langle j\rangle \mid \lambda \in \Lambda, j \in \mathbb{Z}\}$ where $(\Lambda, \prec)$ is a partially ordered finite set. For each $\lambda \in \Lambda$ let

(i) $\mathbb{P}(\lambda)$ denote the projective cover of $\mathbb{L}(\lambda)$,

(ii) $\Delta(\lambda)$ be the maximal quotient of $\mathbb{P}(\lambda)$ such that $[\Delta(\lambda): \mathbb{L}(\mu)]=0$ for all $\mu \succ \lambda$

(iii) $\bar{\Delta}(\lambda)$ be the maximal quotient of $\Delta(\lambda)$ such that $[\operatorname{rad} \bar{\Delta}(\lambda): \mathbb{L}(\mu)]=0$ for all $\mu \succeq \lambda$.

Then $B$ is properly stratified if the following conditions hold for every $\lambda \in \Lambda$ :

(PS1) the kernel of the canonical epimorphism $\mathbb{P}(\lambda) \rightarrow \Delta(\lambda)$ has a filtration with subquotients isomorphic to graded shifts of $\Delta(\mu), \mu \succ \lambda$;

(PS2) the kernel of the canonical epimorphism $\Delta(\lambda) \rightarrow \bar{\Delta}(\lambda)$ has a filtration with subquotients isomorphic to graded shifts of $\bar{\Delta}(\lambda)$;

(PS3) the kernel of the canonical epimorphism $\bar{\Delta}(\lambda) \rightarrow \mathbb{L}(\lambda)$ has a filtration with subquotient isomorphic to graded shifts of $\mathbb{L}(\mu), \mu \prec \lambda$.

The modules $\Delta(i)$ and $\bar{\Delta}(i)$ are called standard and proper standard modules respectively.

Theorem 5.24. For every block $\Gamma$ the algebra $A_{\Gamma}$ is a graded properly stratified algebra. The partially ordered set indexing the simple modules is $(\Gamma, \prec)$. The modules $\Delta(\mu)$ and $\bar{\Delta}(\mu)$ are the standard and proper standard modules respectively. Moreover, the diagonal matrix of the multiplicity numbers of the proper standard modules in the filtrations of the standard modules is a multiple of the identity.

Proof. We already noticed that $A=A_{\Gamma}$ is a finite dimensional associative unital graded algebra over $\mathbb{C}$ with a duality with respect to which the simple modules are self-dual. For $\lambda \in \Gamma$ let $\mathbb{L}(\lambda)=L(\lambda)$ and define $\mathbb{P}(\lambda), \triangle(\lambda)$ and $\bar{\Delta}(\lambda)$ as in Definition 5.23 (i), (ii), (iii) above. By the uniqueness of the projective cover we have $P(\lambda) \cong \mathbb{P}(\lambda)$. From (5.74) and (5.73) we have that $\Delta(\lambda)$ is a quotient of $P(\lambda)$ such that $[\Delta(\lambda): L(\mu)]=0$ for every $\mu \succ \lambda$; from Proposition 5.19 it follows that it is maximal with this property, hence $\Delta(\lambda) \cong \triangle(\lambda)$. By the same argument using (5.73) and Proposition 5.21 we get that $\bar{\Delta}(\lambda) \cong \bar{\Delta}(\lambda)$. Hence we need to show that properties (PS1] 3 ) are satisfied. But this follows immediately from Propositions 5.19, 5.21 and 5.22,

5.5. A bilinear form and self-dual projective modules. We define a bilinear form on $A$ and we determine which projective modules are self-dual.

Defect. Let $\lambda$ be a weight in some block $\Gamma$. We say that a $\wedge$ of $\lambda$ is initial if it has no $\vee$ 's on its left. Let us define the defect of $\lambda$ to be

$$
\operatorname{def}(\lambda)=\#\left\{\text { non-initial } \wedge^{\prime} \text { 's of } \lambda\right\} \text {. }
$$


We have the following elementary result:

Lemma 5.25. The maximal degree of $e_{\lambda} A e_{\lambda}$ is $k(k-1)+2 \operatorname{def}(\lambda)$, and the homogeneous subspace of maximal degree of $e_{\lambda} A e_{\lambda}$ is one dimensional.

Proof. It is straightforward to notice that the homogeneous subspace of maximal degree of $e_{\lambda} A e_{\lambda}$ is one dimensional: the diagram of maximal degree is $\underline{\lambda} \eta^{\sigma} \bar{\lambda}$, where $\eta$ orients every fork of $\underline{\lambda}$ with maximal degree (that is, each $\vee$ is at the rightmost position) and $\sigma$ is the longest element of $\mathbb{S}_{k}$. By definition, the degree of this diagram is obtained by adding $2 \ell(\sigma)$ to the sum of $2(m-1)$ for every $m$-fork of $\underline{\lambda}$. Hence, this degree is $2 \ell(\sigma)$ plus twice the number of non-initial $\wedge$ 's of $\lambda$.

Lemma 5.26. Consider $\lambda, \mu \in \Gamma$ and suppose that $e_{\lambda} A e_{\mu}$ is not trivial. Then the homogeneous subspaces of minimal and maximal degree of $e_{\lambda} A e_{\mu}$ are one dimensional. The minimal and the maximal degrees are, respectively,

$$
\sum_{i=1}^{n-k}\left|\vee_{i}^{\lambda}-\vee_{i}^{\mu}\right| \quad \text { and } \quad k(k-1)+\sum_{i=1}^{n-k}\left|\vee_{i+1}^{\min }-1-\vee_{i}^{\lambda}\right|+\left|\vee_{i+1}^{\min }-1-\vee_{i}^{\mu}\right| \text {, }
$$

where we set $\vee_{i}^{\min }=\min \left\{\vee_{i}^{\lambda}, \vee_{i}^{\mu}\right\}$ and $\vee_{n-k+1}^{\lambda}=n+1$. If $\operatorname{def}(\lambda) \geq \operatorname{def}(\mu)$, then the sum of the two expressions of (5.77) is equal to the maximal degree of $e_{\lambda} A e_{\lambda}$.

Proof. We use the condition (5.6) to determine if a diagram is oriented. The minimal degree diagram is $\lambda \eta^{e} \mu$ where $\vee_{i}^{\eta}=\max \left\{\vee_{i}^{\lambda}, \vee_{i}^{\mu}\right\}$. The maximal degree diagram is $\lambda \eta^{w_{k}} \mu$ where $w_{k} \in \mathbb{S}_{k}$ is the longest element and $\vee_{i}^{\eta}=\min \left\{\vee_{i+1}^{\lambda}, \vee_{i+1}^{\mu}\right\}-1$. Computing their degrees we obtain exactly (5.77).

Let us now check the last assertion. The sum of the two expressions of (5.77) is

$$
k(k-1)+\sum_{i=1}^{n-k} 2\left(\vee_{i+1}^{\min }-1-\vee_{i}^{\min }\right) .
$$

This is the maximal degree of $e_{\eta} A e_{\eta}$ where $\eta \in \Gamma$ is the weight with $\vee_{i}^{\eta}=\vee_{i}^{\text {min }}$. Of course $\operatorname{def}(\eta)=\max \{\operatorname{def}(\lambda), \operatorname{def}(\mu)\}$, and by Lemma 5.25] the maximal degrees of $e_{\lambda} A e_{\lambda}$ and $e_{\eta} A e_{\eta}$ are the same.

Notice that a weight $\lambda$ is of maximal defect if and only if it starts with a $\vee$. If $\lambda$ is not of maximal defect, let $\tilde{\lambda}$ be obtained from $\lambda$ by swapping the first $\vee$ and the first $\wedge$. Otherwise, let $\tilde{\lambda}=\lambda$. In particular, $\tilde{\lambda}$ is always of maximal defect.

Lemma 5.27. For every $\lambda \in \Gamma$ the socle of $P(\lambda)$ contains a degree shift of $L(\tilde{\lambda})$.

In fact, the socle of $P(\lambda)$ is simple; hence it is isomorphic to a degree shift of $L(\tilde{\lambda})$, but we will not need this in what follows.

Proof. It is straightforward to check that the diagram of maximal degree in $A e_{\lambda}$ is of type $\underline{\tilde{\lambda}} \eta^{\sigma} \bar{\lambda}$. The claim follows.

$A$ bilinear form. For every $\lambda \in \Gamma$ of maximal defect, let us choose a non-zero element $\xi_{\lambda}^{\max } \in e_{\lambda} A e_{\lambda}$ of maximal degree (for example, we can choose it to be the diagram $\underline{\lambda} \eta^{\sigma} \bar{\lambda}$ of the previous proof). For every element $z \in A$ write $e_{\lambda} z e_{\lambda}=$ $t \xi_{\lambda}^{\max }+$ terms of lower degree, and set $\Theta_{\lambda}(z)=t$. Moreover, define

$$
\Theta(z)=\sum_{\operatorname{def}(\lambda) \max } \Theta_{\lambda}(z)
$$


Finally, define a bilinear form $\theta: A \times A \rightarrow \mathbb{C}$ by setting $\theta(y, z)=\Theta(y z)$. Obviously, this form is associative in the sense that $\theta(y, z w)=\theta(y z, w)$ for every $y, z, w \in A$.

Lemma 5.28. For every $\lambda$, the form $\theta$ restricted to $e_{\lambda} A e_{\lambda}$ is symmetric and nondegenerate.

Proof. Let $\lambda$ correspond to $z \in D$. Up to a degree shift, $e_{\lambda} A e_{\lambda} \cong \mathrm{Z}_{z, z}$. Since $\mathrm{Z}_{z, z}$ is commutative, note that $\theta$ is symmetric on $e_{\lambda} A e_{\lambda}$. Consider the monomial basis $\left\{1 \mapsto \boldsymbol{x}^{i}\right\}$ that consists of the elements of (4.14) that are not divided by (4.27). It is clear that for every element $\varphi$ in that basis there exists exactly one element $\varphi^{T}$ in the same basis with $\theta\left(\varphi, \varphi^{T}\right) \neq 0$. This proves that the form is non-degenerate.

Let $e_{\mathrm{def}}=\sum_{\operatorname{def}(\lambda) \max } e_{\lambda}$.

Lemma 5.29. The form $\theta$ restricted to $e_{\mathrm{def}} A \times A e_{\mathrm{def}}$ is non-degenerate.

Proof. We may take $t \in e_{\mu} A e_{\lambda}$ for some $\lambda$ of maximal defect and suppose $\theta(y, t)=0$ for every $y \in e_{\lambda} A e_{\mu}$. Let $y_{0}$ be a generator of the minimal-degree subspace of $e_{\lambda} A e_{\mu}$ (which by Lemma 5.26 is one dimensional). In particular, $\theta\left(y^{\prime}, y_{0} t\right)=\theta\left(y^{\prime} y_{0}, t\right)=0$ for every $y^{\prime} \in e_{\lambda} A e_{\lambda}$. By Lemma 5.28, this implies that $y_{0} t=0$. From Lemma 5.30 it follows then that $t=0$.

The reverse follows because $\theta(y, t)=\theta\left(t^{\star}, y^{\star}\right)$.

Lemma 5.30. Suppose $\lambda$ is of maximal defect and let $0 \neq t \in e_{\mu} A e_{\lambda}$. Let also $0 \neq y_{0} \in e_{\lambda} A e_{\mu}$ be of minimal degree. Then $y_{0} t \neq 0$.

Proof. First, let $0 \neq t_{0} \in e_{\mu} A e_{\lambda}$ be of minimal degree, and let us prove that $y_{0} t_{0} \neq 0$. By definition, $y_{0} t_{0}: 1 \mapsto \boldsymbol{x}^{h}$, where $h_{i}=\left|b_{i}^{\lambda}-b_{i}^{\mu}\right| \in\{0,1\}$. First let us suppose that $1 \mapsto \boldsymbol{x}^{\boldsymbol{h}}$ is an element of the basis (4.14), that is, $h_{i}<b_{i}^{\lambda}$ for every $i$. It is quite easy to argue that for every $i$ there exists an index $j$ with $\vee_{i}^{\lambda} \leq j<\vee_{i+1}^{\lambda}$ and $b_{i}^{\lambda}=b_{i}^{\mu}$; in fact it is sufficient to choose $j=\vee_{i}^{\mu}$ if $\vee_{i}^{\mu} \geq \vee_{i}^{\lambda}$ or $j=\vee_{i}^{\lambda}$ otherwise. This means that $1 \mapsto \boldsymbol{x}^{\boldsymbol{h}}$ is not illicit (cf. Theorem 4.17); hence it is not zero.

We should now consider the case in which $1 \mapsto \boldsymbol{x}^{\boldsymbol{h}}$ is not an element of the basis (4.14). This happens if $h_{i}=1$ for some $i$ with $b_{i}^{\lambda}=1$ and $b_{i}^{\mu}=2$. Let $j$ be such that $\vee_{j}^{\lambda}$ is the rightmost $\vee$ in a position $\vee_{j}^{\lambda} \leq i$. It is easy to argue that for $e_{\mu} A e_{\lambda}$ to be non-trivial we must actually have $\vee_{j}^{\lambda}<i$. Let also $i^{\prime}=\vee_{j}^{\max }=\max \left\{\vee_{j}^{\lambda}, \vee_{j}^{\mu}\right\}<i$. Then we have $b_{i^{\prime}}^{\lambda}=b_{i^{\prime}}^{\mu} \geq 2$. Using the relation $h_{1}\left(x_{1}, \ldots, x_{i}\right)=0$ to write $\boldsymbol{x}^{\boldsymbol{h}}$ in our fixed monomial basis we get in particular a term divided by $x_{i^{\prime}}$. Applying the technique of the previous paragraph to this term we get that $y_{0} t_{0} \neq 0$ : the only thing to notice is that $x_{\vee_{j}^{\lambda}} x_{\vee_{j}^{\lambda}+1} \cdots x_{\vee_{j+1}^{\lambda}-1}$ never divides a monomial basis element, since $b_{\vee_{j+1}^{\lambda}-1}^{\lambda}=1$.

Now, it follows from the proof of Lemma 5.28 that there is some element $u \in R$ such that $y_{0} t_{0} u$ generates the maximal degree subspace of $e_{\lambda} A e_{\lambda}$. In particular $y_{0} t_{0} u \neq 0$. By Lemma 5.26, $t_{0} u$ is of maximal degree in $e_{\mu} A e_{\lambda}$. It is then clear by our characterization of $e_{\mu} A e_{\lambda}$ that there exists an element $u^{\prime} \in R$ such that $u^{\prime} t=t_{0} u$. Now $y_{0} t u^{\prime}=y_{0} u^{\prime} t=y_{0} t_{0} u \neq 0$ implies that $y_{0} t \neq 0$.

Self-dual projective modules. Finally, we can determine which indecomposable projective modules are self-dual.

Lemma 5.31. Let $\lambda$ be of maximal defect. Then $P(\lambda)$ is self-dual up to a degree shift. In particular, it is an injective module. 
Proof. By Lemma [5.29, the map $y \longmapsto \theta\left(y^{\star}, \cdot\right)$ defines an isomorphism between $P(\lambda)$ and its dual up to a degree shift.

Theorem 5.32. Let $\lambda \in \Gamma$. Then $P(\lambda)$ is an injective module if and only if $\lambda$ is of maximal defect.

Proof. By Lemma 5.31 if $\lambda$ is of maximal defect, then $P(\lambda)$ is injective. On the other side, suppose $P(\lambda)$ is injective. Then $P(\lambda)$ is a tilting module, and by standard theory it is self-dual (as an ungraded module). In particular, the socle of $P(\lambda)$ is $L(\lambda)$. By Lemma 5.27, $\lambda$ has to be of maximal defect.

Remark 5.33. Let $w^{0}$ be the longest element of $D$. The weights $\lambda$ of maximal defect are exactly the ones that correspond to permutations $w_{k} z, z \in D$, which are in the same right Kazhdan-Lusztig cell of $w_{k} w^{0}$. This can be easily checked using the equivalence between Kazhdan-Lusztig cells and Knuth equivalence (see KL79, §5]) and either applying directly the definition of Knuth equivalence or using its description through the Robinson-Schensted correspondence (cf. Knu73, $\S 5.1 .4$ ] and also Du05]). This gives another proof of a particular case of [MS08, Theorem 5.1] (for the relation with the category $\mathcal{O}$ see Section 6 below).

5.6. Diagrammatic functors $\mathcal{E}_{k}$ and $\mathcal{F}_{k}$. We construct now two functors:

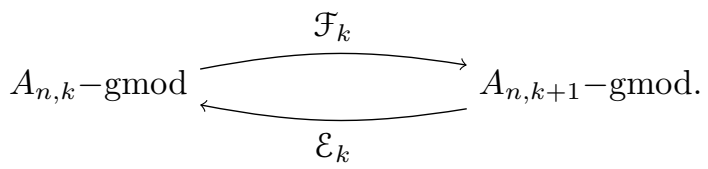

They are important since they are the diagrammatic version of the corresponding functors from Sar13 (see Section 6 below).

Let us fix an integer $n$. For all $k=0, \ldots, n$ let us set in this section $A_{k}=A_{n, k}$. Let $\Gamma_{k}^{\vee}$ be the subset of weights of $\Gamma_{k}$ of maximal defect, and let $\Gamma_{k}^{\wedge}=\Gamma_{k}-\Gamma_{k}^{\vee}$. Notice that given $\lambda \in \Gamma_{k}$ we have $\lambda \in \Gamma_{k}^{\vee}$ if and only if the leftmost symbol of $\lambda$ is a $\vee$, and conversely $\lambda \in \Gamma_{k}^{\wedge}$ if and only if the leftmost symbol of $\lambda$ is a $\wedge$. Let also

$$
e_{k}^{\vee}=\sum_{\lambda \in \Gamma_{k}^{\vee}} e_{\lambda}, \quad e_{k}^{\wedge}=\sum_{\lambda \in \Gamma_{k}^{\wedge}} e_{\lambda} .
$$

In the notation of the previous section, $e_{k}^{\vee}=e_{\mathrm{def}}$.

Consider now $P_{k}^{\vee}=A_{k} e_{k}^{\vee}$, that is, the sum of all indecomposable projectiveinjective $A_{k}$-modules. We want to describe a right $A_{k+1}$ action on it.

For any $\lambda \in \Gamma_{k}^{\vee}$ let $\lambda^{(\wedge)} \in \Gamma_{k+1}^{\wedge}$ be the weight obtained from $\lambda$ by substituting the leftmost symbol, which by assumption is a $\vee$, with a $\wedge$. Conversely, given $\mu \in \Gamma_{k+1}^{\wedge}$ let $\mu^{(\vee)} \in \Gamma_{k}^{\vee}$ be the weight obtained from $\mu$ after substituting the leftmost symbol, which by assumption is a $\wedge$, with a $\vee$. Clearly the map $\lambda \mapsto \lambda^{(\wedge)}$ defines a bijection $\Gamma_{k}^{\vee} \rightarrow \Gamma_{k+1}^{\wedge}$ with inverse $\mu \mapsto \mu^{(\vee)}$.

Lemma 5.34. Let $\lambda, \mu \in A_{k+1}^{\wedge}$. Then we have a natural $R$-module isomorphism

$$
\operatorname{Hom}_{R}\left(\mathrm{C}_{\lambda}, \mathrm{C}_{\mu}\right) \cong \operatorname{Hom}_{R}\left(\mathrm{C}_{\lambda(\mathrm{v})}, \mathrm{C}_{\mu(\mathrm{v})}\right)
$$

that induces a surjective map

$$
e_{\mu} A_{k+1} e_{\lambda} \longrightarrow e_{\mu(\vee)} A_{k} e_{\lambda(\vee)} .
$$


Proof. Since the $\boldsymbol{b}$-sequences of $\lambda$ and $\lambda^{(\vee)}$ are the same, the first claim follows. By Theorem 4.17 the bimodule $\mathrm{W}_{\lambda^{(\vee)}, \mu}(\mathrm{v})$ is generated by $\mathrm{W}_{\lambda, \mu}$ together with the morphism $1 \mapsto x_{1}, \ldots, x_{j}$ where $j=\min \left\{\vee_{1}^{\lambda}, \vee_{1}^{\mu}\right\}$. Hence $e_{\mu(\vee)} A_{k} e_{\lambda(\vee)}$ is a quotient of $e_{\mu} A_{k+1} e_{\lambda}$.

Corollary 5.35. We have a surjective algebra homomorphism

$$
\Psi: e_{k+1}^{\wedge} A_{k+1} e_{k+1}^{\wedge} \rightarrow e_{k}^{\vee} A_{k} e_{k}^{\vee}
$$

Proposition 5.36. We have a well-defined surjective algebra homomorphism

$$
\begin{aligned}
A_{k+1} / A_{k+1} e_{k+1}^{\vee} A_{k+1} & \longrightarrow e_{k}^{\vee} A_{k} e_{k}^{\vee} \\
{[x] } & \longmapsto \Psi\left(e_{k+1}^{\wedge} x e_{k+1}^{\wedge}\right)
\end{aligned}
$$

for $x \in A_{k+1}$, where $\Psi$ is the homomorphism (5.84).

Proof. We need to show that (5.85) does not depend on the particular representative $x$ chosen or equivalently that $\Psi\left(e_{k+1}^{\wedge} x e_{k+1}^{\wedge}\right)=0$ for all $x \in A_{k+1} e_{k+1}^{\vee} A_{k+1}$. By linearity, it suffices to consider the case $x \in A_{k+1} e_{\nu} A_{k+1}$ for $\nu \in \Gamma_{k+1}^{\vee}$. Pick such an $x$ and fix $\lambda, \mu \in \Gamma_{k+1}^{\wedge}$. Choose some morphism $f \in \operatorname{Hom}_{R}\left(\mathrm{C}_{\lambda}, \mathrm{C}_{\mu}\right)$ which corresponds to $e_{\mu} x e_{\lambda}$ in the quotient $\operatorname{Hom}_{R}\left(\mathrm{C}_{\lambda}, \mathrm{C}_{\mu}\right) / \mathrm{W}_{\lambda, \mu}$. Since $x \in A_{k+1} e_{\nu} A_{k+1}$, we can write $f$ as a composition $f_{2} \circ f_{1}$ with $f_{1} \in \operatorname{Hom}_{R}\left(\mathrm{C}_{\lambda}, \mathrm{C}_{\nu}\right)$ and $f_{2} \in \operatorname{Hom}_{r}\left(\mathrm{C}_{\nu}, \mathrm{C}_{\mu}\right)$. By Corollary 4.11. $f_{1}$ is divisible by $x_{1} \cdots x_{\vee_{1}^{\lambda}}$, hence also $f$ is. By Theorem 4.17 (cf. also the proof of Lemma 5.34 above) we have $f \in \mathrm{W}_{\lambda^{(\vee)}, \mu}(\mathrm{v})$, and hence $\Psi\left(e_{\mu} x e_{\lambda}\right)=0$. Since $\lambda$ and $\mu$ were chosen arbitrarily in $\Gamma_{k+1}^{\wedge}$, it follows that $\Psi\left(e_{k+1}^{\wedge} x e_{k+1}^{\wedge}\right)=0$.

The surjectivity of (5.85) is a direct consequence of the surjectivity of (5.84).

The functor $\mathcal{F}_{k}$. Let us now define $\mathbf{F}_{k}$ to be the $\left(A_{k}, A_{k+1}\right)$-bimodule $P_{k}^{\vee}$, where the right $A_{k+1}$-structure is induced by the quotient map $A_{k+1} \rightarrow A_{k+1} / A_{k+1} e_{k+1}^{\vee} A_{k+1}$ composed with (5.85). The bimodule $\mathbf{F}_{k}$ defines a right-exact functor

$$
\mathcal{F}_{k}: A_{k+1}-\operatorname{gmod} \stackrel{\mathbf{F}_{k} \otimes A_{k+1}}{\longrightarrow} A_{k}-\operatorname{gmod} .
$$

For each indecomposable projective module $P(\mu)=A_{k+1} e_{\mu}$ we have

$$
\mathbf{F}_{k} \otimes_{A_{k+1}}\left(A_{k+1} e_{\mu}\right)= \begin{cases}A_{k} e_{\lambda} & \text { if } \lambda^{(\wedge)}=\mu \text { for some } \lambda \in \Gamma_{k}, \\ 0 & \text { otherwise. }\end{cases}
$$

The functor $\mathcal{E}_{k}$. The usual hom-tensor adjunction gives a natural isomorphism

$$
\operatorname{Hom}_{A_{k}}\left(\mathbf{F}_{k} \otimes_{A_{k+1}} M, N\right) \cong \operatorname{Hom}_{A_{k+1}}\left(M, \operatorname{Hom}_{A_{k}}\left(\mathbf{F}_{k}, N\right)\right)
$$

for all $M \in A_{k+1}$-gmod, $N \in A_{k}$-gmod. Notice that we have a natural isomorphism $\operatorname{Hom}_{A_{k}}\left(\mathbf{F}_{k}, N\right) \cong \operatorname{Hom}_{A_{k}}\left(\mathbf{F}_{k}, A_{k}\right) \otimes_{A_{k}} N$, where $\operatorname{Hom}_{A_{k}}\left(\mathbf{F}_{k}, A_{k}\right)$ is regarded as an $\left(A_{k+1}, A_{k}\right)$-bimodule. Let us therefore define $\mathbf{E}_{k}$ to be the $\left(A_{k+1}, A_{k}\right)$ bimodule $\operatorname{Hom}_{A_{k}}\left(\mathbf{F}_{k}, A_{k}\right)$, so that the functor

$$
\varepsilon_{k}: A_{k}-\operatorname{gmod} \stackrel{\mathbf{E}_{k} \otimes_{A_{k}}}{\longrightarrow} A_{k+1}-\operatorname{gmod}
$$

is right adjoint to $\mathcal{F}_{k}$. Since $\mathbf{F}_{k}$ is a projective $A_{k}$-module the functor $\mathcal{E}_{k}$ is exact.

Remark 5.37. Since $\mathbf{F}_{k}=A_{k} e_{k}^{\vee}$ as a left $A_{k}$-module, we have $\operatorname{Hom}_{A_{k}}\left(\mathbf{F}_{k}, A_{k}\right) \cong$ $e_{k}^{\vee} A_{k}$ as a right $A_{k}$-module. Hence $\mathbf{E}_{k}$ is the $\left(A_{k+1}, A_{k}\right)$-bimodule obtained from $\mathbf{F}_{k}$ by turning the left $A_{k}$-action (resp. the right $A_{k+1}$-action) into a right (resp. left) one using the anti-isomorphism $\star$ (5.28) of $A_{k}$ (resp. $A_{k+1}$ ). Specifically, the 
left action of $\alpha \in A_{k+1}$ on $y \in \mathbf{E}_{k}$ is given by $\alpha \cdot y=y \alpha^{\star}$, and the right action of $\beta \in A_{k}$ is given by $y \cdot \beta=\beta^{\star} y$.

\section{Category $\mathcal{O}$}

We recall now the definition of the BGG category $\mathcal{O}\left(\mathfrak{g l}_{n}\right)$ and of its subquotient categories $\mathcal{O}_{0}^{\mathfrak{p}, \mathfrak{q} \text {-pres }}$ from Sar13]. We start with some general facts about Serre subcategories and Serre quotient categories.

6.1. Serre subcategories and Serre quotients. Let $\mathcal{A}$ be some abelian category which is equivalent to the category of finitely generated modules over some finite dimensional $\mathbb{C}$-algebra. Let $L(\lambda)$ for $\lambda \in \Lambda$ be the simple objects of $\mathcal{A}$ up to isomorphism. For all $\lambda \in \Lambda$ let $P(\lambda)$ be the projective cover of $L(\lambda)$. Let $P=$ $\bigoplus_{\lambda \in \Lambda} P(\lambda)$ be a minimal projective generator and let $R=\operatorname{End}_{\mathcal{A}}(P)$. Then we have an equivalence of categories $\mathcal{A} \cong \bmod -R$ via the functor $\operatorname{Hom}_{\mathcal{A}}(P,-)$.

Serre subcategories. For each subset $\Gamma \subseteq \Lambda$ define $\mathcal{S}_{\Gamma}$ to be the Serre subcategory of $\mathcal{A}$ consisting of the modules with all composition factors of type $L(\gamma)$ for $\gamma \in \Gamma$. Let $I_{\Gamma}$ be the two-sided ideal of $R=\operatorname{End}_{\mathcal{A}}(P)$ generated by all endomorphisms which factor through some $P(\eta)$ for $\eta \notin \Gamma$. Then

$$
\mathcal{S}_{\Gamma} \cong \bmod -R / I_{\Gamma}
$$

Notice that if we let $e_{\lambda}$ for $\lambda \in \Lambda$ be the idempotent projecting onto $\operatorname{End}_{\mathcal{A}}(P(\lambda)) \subset$ $R$ and $e_{\Gamma}^{\perp}=\sum_{\eta \notin \Gamma} e_{\eta}$, then $I_{\Gamma}=R e_{\Gamma}^{\perp} R$.

A complete set of pairwise non-isomorphic simple objects in $\mathcal{S}_{\Gamma}$ is given by the $L(\gamma)$ 's for $\gamma \in \Gamma$ and each of them has a projective cover $P_{\mathcal{S}_{\Gamma}}(\gamma)$ in $\mathcal{S}_{\Gamma}$, which is the biggest quotient of $P(\gamma)$ which lies in $\mathcal{S}_{\Gamma}$.

Serre quotients. Let us denote by $\mathcal{A} / \mathcal{S}_{\Gamma}$ the Serre quotient category. Analogously as above, we have an equivalence of categories

$$
\mathcal{A} / \mathcal{S}_{\Gamma} \cong \bmod -\operatorname{End}_{\mathcal{A}}\left(P_{\Gamma}\right),
$$

where $P_{\Gamma}=\bigoplus_{\eta \in \Lambda-\Gamma} P(\eta)$ (see for example [AM11, Prop. 33]). The quotient functor is $Q=\operatorname{Hom}_{\mathcal{A}}\left(P_{\Gamma},-\right)$. Notice that $\operatorname{End}_{\mathcal{A}}\left(P_{\Gamma}\right)=e_{\Gamma} R e_{\Gamma}$ where $e_{\Gamma}=\sum_{\gamma \in \Gamma} e_{\gamma}$.

A complete set of pairwise non-isomorphic simple objects in $\mathcal{A} / \mathcal{S}_{\Gamma}$ is given by the $L(\eta)$ 's for $\eta \in \Lambda-\Gamma$, with projective covers $P(\eta)$.

Remark 6.1 . The category $\mathcal{A} / \mathcal{S}_{\Gamma}$ is also equivalent to the category of $\operatorname{Add}\left(P_{\Gamma}\right)-$ presentable modules, that is, the full subcategory of $\mathcal{A}$ consisting of all modules $M \in \mathcal{A}$ having a presentation

$$
Q_{1} \longrightarrow Q_{2} \longrightarrow M
$$

with $Q_{1}, Q_{2} \in \operatorname{Add}\left(P_{\Gamma}\right)$. Here $\operatorname{Add}\left(P_{\Gamma}\right)$ is the additive full subcategory of $\mathcal{A}$ consisting of all objects which admit a direct sum decomposition with summands being direct summands of $P_{\Gamma}$. 
6.2. Category $\mathcal{O}$. Let $\mathfrak{g l}_{n}$ be the general Lie algebra of complex $n \times n$ matrices. Denote by $\mathfrak{h}$ the Cartan subalgebra of all diagonal matrices and by $\mathfrak{b}=\mathfrak{h} \oplus \mathfrak{n}^{+}$the Borel subalgebra of all upper triangular matrices. Let $\alpha_{1}, \ldots, \alpha_{n-1}$ be the simple roots. We identify the Weyl group with $\mathbb{S}_{n}$.

The BGG category $\mathcal{O}=\mathcal{O}\left(\mathfrak{g l}_{n}\right)=\mathcal{O}\left(\mathfrak{g l}_{n} ; \mathfrak{b}\right)$, introduced in BGG76, is the full subcategory of all $\mathfrak{g l}_{n}$-modules $M$ which are

(O1) finitely generated;

$(\mathcal{O} 2)$ a direct sum of weight spaces for the action of $\mathfrak{h}$;

(O3) locally $\mathfrak{n}^{+}$-finite (that is, for every $x \in M$ the $\mathfrak{n}^{+}$-submodule generated by $x$ is finite dimensional).

The category $\mathcal{O}$ decomposes into blocks. Let $\mathcal{O}_{0}$ be the block containing the trivial representation $L(0)$. Then $\mathcal{O}_{0}$ is a highest weight category with simple modules $L(w \cdot 0)$ for $w \in \mathbb{S}_{n}$. In particular, each simple module $L(w \cdot 0)$ has a projective cover $P(w \cdot 0)$. For each $w$ in $\mathbb{S}_{n}$ there is a universal highest weight module $M(w \cdot 0) \in \mathcal{O}_{0}$ with highest weight $w \cdot 0$, usually called a Verma module. The projective module $P(w \cdot 0)$ is also the projective cover of $M(w \cdot 0)$.

If we let $P=\bigoplus_{w \in \mathbb{S}_{n}} P(w \cdot 0)$ be a minimal projective generator, then we have $\mathcal{O}_{0} \cong \bmod -R$ where $R=\operatorname{End}_{\mathcal{O}}(P)$. For more details about $\mathcal{O}$ we refer to Hum08.

Parabolic category $\mathcal{O}$. Fix a standard parabolic subalgebra $\mathfrak{p} \subseteq \mathfrak{g l}_{n}$ corresponding to a parabolic subgroup $W_{\mathfrak{p}} \subseteq \mathbb{S}_{n}$. Let $W^{\mathfrak{p}}$ denote the shortest coset representatives for $W_{\mathfrak{p}} \backslash \mathbb{S}_{n}$. Then the parabolic category $\mathcal{O}_{0}^{\mathfrak{p}}$ is the Serre subcategory of $\mathcal{O}_{0}$ generated by all simple modules of the type $L(w \cdot 0)$ for $w \in W^{\mathfrak{p}}$. As in (6.1), if we let $I_{\mathfrak{p}}$ be the two-sided ideal of $\operatorname{End}_{\mathcal{O}}(P)$ generated by all morphisms which factor through some $P(z \cdot 0)$ for $z \notin W^{\mathfrak{p}}$, then

$$
\mathcal{O}_{0}^{\mathfrak{p}} \cong \bmod -\operatorname{End}_{\mathcal{O}}(P) / I_{\mathfrak{p}}
$$

$\mathfrak{q}$-presentable category $\mathcal{O}$. Let $\mathfrak{q} \subseteq \mathfrak{g l}_{n}$ also be a standard parabolic subalgebra corresponding to a parabolic subgroup $W_{\mathfrak{q}} \subseteq \mathbb{S}_{n}$. Let as before $W^{\mathfrak{q}}$ denote the shortest coset representatives for $W_{\mathfrak{q}} \backslash \mathbb{S}_{n}$, and let $w_{\mathfrak{q}} \in W_{\mathfrak{q}}$ be the longest element, so that $w_{\mathfrak{q}} W^{\mathfrak{q}}$ is the set of the longest coset representatives. Let $\mathcal{S}^{\mathfrak{q}}$ be the Serre subcategory of $\mathcal{O}_{0}$ generated by all simple modules $L(z \cdot 0)$ for $z \notin w_{\mathfrak{q}} W^{\mathfrak{q}}$. Then the $\mathfrak{q}$-presentable category $\mathcal{O}_{0}^{\mathfrak{q} \text {-pres }}$ is the Serre quotient $\mathcal{O}_{0} / \mathcal{S}^{\mathfrak{q}}$ (the choice of this name is motivated by Remark 6.1). As in (6.2), if we let $P_{\mathfrak{q}}=\bigoplus_{w \in w_{\mathfrak{q}} W_{\mathfrak{q}}} P(w \cdot 0)$, then we have an equivalence of categories

$$
\mathcal{O}_{0}^{\mathfrak{q} \text {-pres }} \cong \bmod -\operatorname{End}_{\mathcal{O}}\left(P_{\mathfrak{q}}\right) .
$$

Remark 6.2. Note that for $w, z \in w_{\mathfrak{q}} W^{\mathfrak{q}}$ we have $\operatorname{Hom}_{\mathcal{O}}(P(w \cdot 0), P(z \cdot 0))=$ $\operatorname{Hom}_{\mathcal{O}_{0}^{\mathfrak{q}-p r e s}}(P(w \cdot 0), P(z \cdot 0))$, since both the head of $P(w \cdot 0)$ and the socle of $P(z \cdot 0)$ are simple modules which are not in $\mathcal{S}^{\mathfrak{q}}$. For this it is essential that we are working with longest coset representatives.

Mixed parabolic and $\mathfrak{q}$-presentable category $\mathcal{O}$. It is possible to mix the two constructions above for two parabolic subalgebras $\mathfrak{p}, \mathfrak{q} \subseteq \mathfrak{g l}_{n}$ which are orthogonal in the sense that the corresponding sets of simple roots are disjoint and hence $W_{\mathfrak{p}} \times W_{\mathfrak{q}}$ is a subgroup of $\mathbb{S}_{n}$. One can consider the Serre subcategory $\mathcal{S}$ of $\mathcal{O}_{0}^{\mathfrak{p}}$ generated by all simple modules of the type $L(z \cdot 0)$ for $z \in W^{\mathfrak{p}}, z \notin w_{\mathfrak{q}} W^{\mathfrak{q}}$ and then define $\mathcal{O}_{0}^{\mathfrak{p}, \mathfrak{q} \text {-pres }}$ to be the Serre quotient $\mathcal{O}_{0}^{\mathfrak{p}} / \mathcal{S}$. Alternatively, one can reverse the construction and define $\mathcal{O}_{0}^{\mathfrak{p}, \mathfrak{q} \text {-pres }}$ to be the Serre subcategory of $\mathcal{O}_{0}^{\mathfrak{q}-\text { pres }}$ generated by 
all simple modules of the form $L(w \cdot 0)$ for $w \in w_{q} W^{\mathfrak{q}} \cap W^{\mathfrak{p}}$. As one expects, the two definitions agree (see [Sar13]).

To get an explicit description of $\mathcal{O}_{0}^{\mathfrak{p}, \mathfrak{q} \text {-pres }}$, let $P_{\mathfrak{q}}^{\mathfrak{p}}=\bigoplus_{w \in w_{q} W^{\mathfrak{q}} \cap W^{\mathfrak{p}}} P^{\mathfrak{p}}(w \cdot 0)$, where $P^{\mathfrak{p}}(w \cdot 0)$ is the projective cover of $L(w \cdot 0)$ in $\mathcal{O}_{0}^{\mathfrak{p}}$. Let moreover $\bar{I}_{\mathfrak{p}}$ be the two-sided ideal of $\operatorname{End}_{\mathcal{O}}\left(P_{\mathfrak{q}}\right)$ generated by all endomorphisms which factor through some $P(z \cdot 0)$ for $z \in w_{\mathfrak{q}} W^{\mathfrak{q}}, z \notin W^{\mathfrak{p}}$. Then we have equivalences of categories

$$
\mathcal{O}_{0}^{\mathfrak{p}, \mathfrak{q} \text {-pres }} \cong \bmod -\operatorname{End}_{\mathcal{O}}\left(P_{\mathfrak{q}}^{\mathfrak{p}}\right) \cong \bmod -\operatorname{End}_{\mathcal{O}}\left(P_{\mathfrak{q}}\right) / \bar{I}_{\mathfrak{p}}
$$

6.3. Soergel's theorems. Fix a positive integer $n$. Using the notation of Section 4. we recall the main results from [Soe90.

Theorem 6.3 (Soe90). Let $w_{n}$ be the longest element of $\mathbb{S}_{n}$. We have a canonical isomorphism $\operatorname{End}_{\mathcal{O}}\left(P\left(w_{n} \cdot 0\right)\right) \cong B$. The functor $\mathbb{V}=\operatorname{Hom}_{\mathcal{O}}\left(P\left(w_{n} \cdot 0\right),-\right): \mathcal{O}_{0} \rightarrow$ $B-\bmod$ is fully faithful on projective modules. Moreover, for each $z \in \mathbb{S}_{n}$ the $B$-module $\mathbb{V P}(z \cdot 0)$ is isomorphic to the Soergel module $\mathrm{C}_{z}$ defined in $\$ 4.1$,

In particular, it follows from Soergel's result and the discussion in 6.2 that $\mathcal{O}_{0} \cong \bmod -\operatorname{End}_{B}\left(\bigoplus_{z \in \mathbb{S}_{n}} C_{z}\right)$. As explained in 4.4 there is a natural way to consider the Soergel modules as graded modules. It then makes sense to define the graded version of the category $\mathcal{O}$ to be

$$
{ }^{\mathbb{Z}} \mathcal{O}_{0}=\operatorname{gmod}-\operatorname{End}_{B}\left(\bigoplus_{z \in \mathbb{S}_{n}} C_{z}\right) .
$$

This is the Koszul grading of $\mathcal{O}_{0}$ BGS96. Analogously one can define graded versions of the categories $\mathcal{O}_{0}^{\mathfrak{p}}, \mathcal{O}_{0}^{\mathfrak{q} \text {-pres }}, \mathcal{O}_{0}^{\mathfrak{p}, \mathfrak{q} \text {-pres }}$.

We prove now a technical lemma we used in Section 4.

Lemma 6.4. The module $\mathrm{C}_{z}$ is cyclic (generated by $1 \otimes \cdots \otimes 1$ ) if and only if $\mathcal{P}_{e, z}=v^{\ell(z)}$, i.e., if and only if $H_{e}$ appears exactly once with coefficient $v^{\ell(z)}$ in the expression of the canonical basis element $C_{z}$.

Proof. Let $\mathcal{P}_{e, z}$ be the Kazhdan-Lusztig polynomial which gives the coefficient of $H_{e}$ in the expression of $C_{z}$ in the standard basis. Let $(P(z \cdot 0): M(0))$ denote the multiplicity of the dominant Verma module $M(0)$ in some Verma flag of the indecomposable projective module $P(z \cdot 0)$ in the category $\mathcal{O}\left(\mathfrak{g l}_{n}\right)$. By the KazhdanLusztig conjecture we have $\left.\mathcal{P}_{e, z}\right|_{v=1}=(P(z \cdot 0): M(0))$. By [Str03, Lemma 7.3], $(P(z \cdot 0): M(0))$ is the cardinality of a minimal system of generators for $\mathrm{C}_{z}$.

6.4. The category corresponding to the algebra $A_{n, k}$. For this section, fix two integers $n \geq 0$ and $0 \leq k \leq n$. Let $W_{k}, W_{k}^{\perp}$ be the parabolic subgroups of $\mathbb{S}_{n}$ defined in 3.2. Let $\mathfrak{q}, \mathfrak{p} \subseteq \mathfrak{g l}_{n}$ be the standard parabolic subalgebras with $W_{\mathfrak{q}}=W_{k}$ and $W_{\mathfrak{p}}=W_{k}^{\perp}$ so that $W_{\mathfrak{q}} \times W_{\mathfrak{p}}=\mathbb{S}_{k} \times \mathbb{S}_{n-k} \subseteq \mathbb{S}_{n}$. We remark that the resulting category ${ }^{\mathbb{Z}} \mathcal{O}_{0}^{\mathfrak{p}, \mathfrak{q} \text {-pres }}$ is the category denoted by $\mathcal{Q}_{k}(\mathfrak{m})$ in Sar13.

As in Section 4 , let $D$ be the set of shortest coset representatives for $\mathbb{S}_{k} \times \mathbb{S}_{n-k} \backslash \mathbb{S}_{n}$, that is, $D=W^{\mathfrak{q}} \cap W^{\mathfrak{p}}$. Recall Definition 4.13 for illicit morphisms.

Proposition 6.5. We have an equivalence of categories

$$
\mathcal{O}_{0}^{\mathfrak{p}, \mathfrak{q} \text {-pres }} \cong \bmod -\left(\text { End }\left(\bigoplus_{z \in D} \mathrm{C}_{w_{k} z}\right) /\{\text { illicit morphisms }\}\right) \text {. }
$$


In particular, we can define the graded version ${ }^{\mathbb{Z}} \mathcal{O}_{0}^{\mathfrak{p}, \mathfrak{q} \text {-pres }}$ of $\mathcal{O}_{0}^{\mathfrak{p}, \mathfrak{q} \text {-pres }}$ to be

$$
{ }^{\mathbb{Z}} \mathcal{O}_{0}^{\mathfrak{p}, \mathfrak{q} \text {-pres }}=\operatorname{gmod}-\left(\text { End }\left(\bigoplus_{z \in D} \mathrm{C}_{w_{k} z}\right) /\{\text { illicit morphisms }\}\right) .
$$

Proof. By the discussion above we have $\mathcal{O}_{0}^{\mathfrak{p}, \mathfrak{q} \text {-pres }} \cong \bmod -\operatorname{End}_{\mathcal{O}}\left(P_{\mathfrak{q}}\right) / \bar{I}_{\mathfrak{p}}$. Here $P_{\mathfrak{q}}=\bigoplus_{w \in w_{\mathfrak{q}} W_{\mathfrak{q}}} P(w \cdot 0)$. Since $\operatorname{End}_{\mathcal{O}}\left(P_{\mathfrak{q}}\right)=\bigoplus_{w, z \in w_{\mathfrak{q}} W_{\mathfrak{q}}} \operatorname{Hom}(P(w \cdot 0), P(z \cdot 0))$ and any morphisms with source or target some $P(y \cdot 0)$ for $y \notin W^{\mathfrak{p}}$ lies in the ideal $\bar{I}_{\mathfrak{p}}$, we have

$$
\mathcal{O}_{0}^{\mathfrak{p}, \mathfrak{q} \text {-pres }} \cong \bmod -\operatorname{End}_{\mathcal{O}}\left(\bigoplus_{w \in D} P\left(w_{\mathfrak{q}} w \cdot 0\right)\right) / \bar{I}_{\mathfrak{p}}
$$

After applying the isomorphism induced by the functor $\mathbb{V}$, the ideal $\bar{I}_{\mathfrak{p}}$ becomes exactly the ideal generated by illicit morphisms. Hence the claim follows from Theorem 6.3 .

If $Q\left(w_{k} z\right)$ is the projective cover of the simple module $L\left(w_{k} z \cdot 0\right)$ for all $z \in D$, it follows also that

$$
\operatorname{Hom}\left(Q\left(w_{k} z\right), Q\left(w_{k} z^{\prime}\right)\right) \cong \operatorname{Hom}_{R}\left(\mathrm{C}_{w_{k} z}, \mathrm{C}_{w_{k} z^{\prime}}\right) / \mathrm{W}_{z, z^{\prime}}=\mathrm{Z}_{z, z^{\prime}} .
$$

We deduce then:

Lemma 6.6. Let $z, z^{\prime} \in D$, and let $\lambda, \mu \in \Gamma$ be the corresponding weights. The dimension of $\mathrm{Z}_{z, z^{\prime}}$ is $k$ ! times the number of unenhanced weights $\eta$ such that $\underline{\mu} \eta \bar{\lambda}$ is oriented.

Proof. We have $\mathbf{Z}_{z, z^{\prime}} \cong \operatorname{Hom}_{\mathcal{O}_{0}^{\mathfrak{p}, \mathfrak{q}-\text { pres }}}\left(Q\left(w_{k} z\right), Q\left(w_{k} z^{\prime}\right)\right)$. The dimension of this homomorphism space is computed in [Sar13, Lemma 7.13] in terms of evaluation of canonical basis diagrams labeled by standard basis diagrams. This translates immediately in terms of oriented fork diagrams (notice that a canonical basis diagram of Sar13 is the same as a lower fork diagram and a standard basis diagram of Sar13] is the same as an unenhanced weight).

Theorem 6.7. Let $Q_{\mathfrak{q}}^{\mathfrak{p}}=\bigoplus_{x \in D} Q\left(w_{k} x\right)$ be the sum of all indecomposable projective modules (up to isomorphism) in ${ }^{\mathbb{Z}} \mathcal{O}_{0}^{\mathfrak{p}, \mathfrak{q} \text {-pres }}$. Then we have an isomorphism of

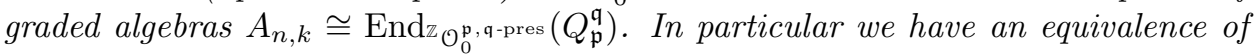
categories

$$
\operatorname{gmod}-A_{n, k} \cong{ }^{\mathbb{Z}} \mathcal{O}_{0}^{\mathfrak{p}, \mathfrak{q} \text {-pres }}
$$

Proof. We just need to identify the quotient of the endomorphism algebra appearing in the r.h.s. of (6.9) with $A_{n, k}$. This follows from Corollary 5.11,

In Section 5 we focused on left $A_{n, k}$-modules, but the whole section could be rewritten for right modules. Alternatively, since the algebra $A_{n, k}$ has an antiautomorphism $\star(5.28)$, the categories of right and left graded $A_{n, k}$-modules are equivalent. Hence we actually have an equivalence

$$
A_{n, k}-\operatorname{gmod} \cong{ }^{\mathbb{Z}} \mathcal{O}_{0}^{\mathfrak{p}, \mathfrak{q} \text {-pres }} .
$$

Although perhaps the equivalence (6.12) is conceptually the right one, we personally prefer to work with left $A_{n, k}$-modules. 
6.5. The functors $\mathcal{F}_{k}$ and $\mathcal{E}_{k}$. We now want to relate the diagrammatic functors $\mathcal{F}_{k}$ and $\mathcal{E}_{k}$ defined in $\$ 5.6$ with their Lie theoretical versions from [Sar13], of which we briefly recall the definition.

Fix two integers $n>0$ and $0 \leq k<n$. Let $W_{k}, W_{k}^{\perp}, W_{k+1}, W_{k+1}^{\perp}$ be the parabolic subgroups of $\mathbb{S}_{n}$ defined in 3.2 Let $\mathfrak{q}, \mathfrak{p} \subseteq \mathfrak{g l}_{n}$ be the standard parabolic subalgebras with $W_{\mathfrak{q}}=W_{k}$ and $W_{\mathfrak{p}}=W_{k}^{\perp}$, and let $\mathfrak{q}^{\prime}, \mathfrak{p}^{\prime} \subseteq \mathfrak{g l}_{n}$ be the standard parabolic subalgebras with $W_{\mathfrak{q}^{\prime}}=W_{k+1}$ and $W_{\mathfrak{p}^{\prime}}=W_{k+1}^{\perp}$. Notice that $\mathfrak{p}^{\prime} \subset \mathfrak{p}$ and $\mathfrak{q} \subset \mathfrak{q}^{\prime}$

Let $H=\operatorname{End}_{\mathcal{O}}\left(P_{\mathfrak{q}}^{\mathfrak{p}^{\prime}}\right)$, where $P_{\mathfrak{q}}^{\mathfrak{p}^{\prime}}$ is a minimal projective generator of $\mathbb{Z}_{\mathcal{O}_{0}^{\mathfrak{p}^{\prime}}, \mathfrak{q} \text {-pres }}$. Let also

- $f_{\mathfrak{q}^{\prime}} \in H$ be the idempotent projecting onto the direct sum of the projective modules $P^{\mathfrak{p}^{\prime}}(x \cdot 0)$ for $x \in w_{\mathfrak{q}^{\prime}} W^{\mathfrak{q}^{\prime}} \cap W^{\mathfrak{p}^{\prime}}$,

- $e^{\mathfrak{p}} \in H$ be the idempotent projecting onto the direct sum of the indecomposable projective modules $P^{\mathfrak{p}^{\prime}}(x \cdot 0) \in{ }^{\mathbb{Z}} \mathcal{O}_{0}^{\mathfrak{p}^{\prime}, \mathfrak{q} \text {-pres }}$ for $x \in w_{\mathfrak{q}^{\prime}} W^{\mathfrak{q}^{\prime}} \cap W^{\mathfrak{p}}$ but $x \notin w_{\mathfrak{q}} W^{\mathfrak{q}}$.

Then we have by the discussion in 86.2 (using the transitive property of taking parabolic subcategories and presentable quotient categories; see [Sar13]):

$$
A_{k} \cong H / H e^{\mathfrak{p}} H \quad \text { and } \quad A_{k+1} \cong f_{\mathfrak{q}^{\prime}} H f_{\mathfrak{q}^{\prime}} .
$$

We have a diagram

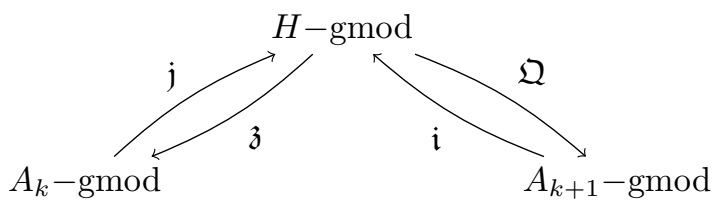

where

- $\mathfrak{j}=\operatorname{Res}_{H}^{A_{k}}$ is the restriction functor induced by the projection map $H \rightarrow$ $H / H e^{\mathfrak{p}} H \cong A_{k}$

- $\mathfrak{z}=\left(H / H e^{\mathfrak{p}} H\right) \otimes_{H}$ is the left adjoint of $\mathfrak{j}$;

- $\mathfrak{Q}=\operatorname{Res}_{A_{k+1}}^{H}$ is the restriction functor induced by the inclusion $A_{k+1} \cong$ $f_{\mathfrak{q}^{\prime}} H f_{\mathfrak{q}^{\prime}} \hookrightarrow H$

- $\mathfrak{i}=H \otimes_{f_{\mathfrak{q}^{\prime}} H f_{\mathfrak{q}^{\prime}}}$ is the left adjoint of $\mathfrak{Q}$.

Let us define $\mathcal{E}_{k}=\mathfrak{Q} \circ \mathfrak{j}$ and $\mathcal{F}_{k}=\mathfrak{z} \circ \mathfrak{i}$. We get then a pair of adjoint functors $\left(\mathcal{F}_{k}, \mathcal{E}_{k}\right)$ :

$$
A_{k}-\operatorname{gmod} \underset{\mathcal{F}_{k}}{\stackrel{\mathcal{E}_{k}}{\longrightarrow}} A_{k+1}-\operatorname{gmod}
$$

Remark 6.8. Under the equivalences of categories

$$
\begin{aligned}
A_{k}-\operatorname{gmod} & \cong{ }^{\mathbb{Z}} \mathcal{O}_{0}^{\mathfrak{p}, \mathfrak{q} \text {-pres }}, \\
H-\operatorname{gmod} & \cong{ }^{\mathbb{Z}} \mathcal{O}_{0}^{\mathfrak{p}^{\prime}, \mathfrak{q} \text {-pres }}, \\
A_{k+1}-\operatorname{gmod} & \cong{ }^{\mathbb{Z}} \mathcal{O}_{0}^{\mathfrak{p}^{\prime}, \mathfrak{q}^{\prime} \text {-pres },}
\end{aligned}
$$


the functors just defined have a Lie theoretical interpretation: $\mathfrak{i}$ and $\mathfrak{j}$ are inclusion of subcategories, $\mathfrak{z}$ is a Zuckermann's functor and $\mathfrak{Q}$ a coapproximation functor; see Sar13.

Proposition 6.9. The functor $\mathcal{F}_{k}$ is naturally isomorphic to the functor $\mathbf{F}_{k} \otimes_{A_{k+1}}$.

Proof. The functor $\mathcal{F}_{k}$ is defined by

$$
M \longmapsto\left(H / H e^{\mathfrak{p}} H\right) \otimes_{f_{\mathfrak{q}^{\prime}} H f_{\mathfrak{q}^{\prime}}} M,
$$

which is the same as

$$
M \longmapsto\left(H / H e^{\mathfrak{p}} H\right) \bar{f}_{\mathfrak{q}^{\prime}} \otimes_{f_{\mathfrak{q}^{\prime}} H f_{\mathfrak{q}^{\prime}}} M,
$$

where $\bar{f}_{\mathfrak{q}^{\prime}}$ is the image of $f_{\mathfrak{q}^{\prime}}$ in $H / H e^{\mathfrak{p}} H$. Obviously $\left(H / H e^{\mathfrak{p}} H\right) \bar{f}_{\mathfrak{q}^{\prime}}=P_{k}^{\vee}$ is a left $A_{k}$-module. It is easy to notice that also the right $A_{k+1}$-module structure is the same, since in both cases it is the natural structure induced by the bigger algebra $\operatorname{End}_{\mathcal{O}}(P)$, where $P$ is a minimal projective generator of ${ }^{\mathbb{Z}} \mathcal{O}_{0}$.

By the uniqueness of the adjoint functor we also get:

Proposition 6.10. The functor $\mathcal{E}_{k}$ is naturally isomorphic to the functor $\mathbf{E}_{k} \otimes_{A_{k}}$.

Consider now $\mathcal{E}_{k}$ as an object in the category of functors between $A_{k}-$ gmod and $A_{k+1}-$ gmod, and $\mathcal{F}_{k}$ as an object in the category of functors between $A_{k+1}-\operatorname{gmod}$ and $A_{k}$-gmod. As such, we can compute their endomorphism rings:

Theorem 6.11. We have $\operatorname{End}\left(\mathcal{E}_{k}\right) \cong \operatorname{End}\left(\mathcal{F}_{k}\right) \cong \mathbb{C}\left[x_{1}, \ldots, x_{n}\right] / I_{k}$ where $I_{k}$ is the ideal generated by the complete symmetric functions

$$
\begin{aligned}
h_{k+1}\left(x_{i_{1}}, \ldots, x_{i_{m}}\right) & \text { for all } & 1 & \leq m \leq n-k, \\
h_{n-m+1}\left(x_{i_{1}}, \ldots, x_{i_{m}}\right) & \text { for all } & n-k+1 & \leq m \leq n .
\end{aligned}
$$

In particular, $\mathcal{E}_{k}$ and $\mathcal{F}_{k}$ are indecomposable functors.

Proof. Let us first compute $\operatorname{End}\left(\mathcal{F}_{k}\right)$. By Proposition $\left[6.9\right.$, we have $\operatorname{End}\left(\mathcal{F}_{k}\right) \cong$ End $_{A_{k} \otimes A_{k+1}^{\text {op }}}\left(\mathbf{F}_{k}\right)$. Since the structure of a right $A_{k+1}-$ module is induced by the

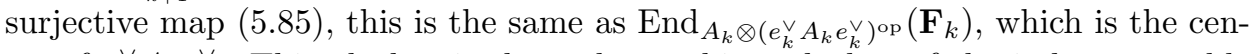
ter of $e_{k}^{\vee} A_{k} e_{k}^{\vee}$. This algebra is the endomorphism algebra of the indecomposable projective-injective modules of ${ }^{\mathbb{Z}} \mathcal{O}_{0}^{\mathfrak{p}, \mathfrak{q} \text {-pres }}$, where as before $\mathfrak{p}, \mathfrak{q} \subseteq \mathfrak{g l}_{n}$ are the parabolic subalgebras corresponding to $k$. Since the projective-injective modules of

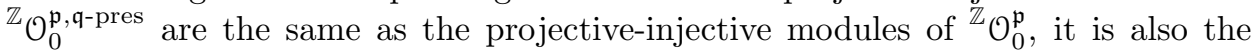
endomorphism algebra of the indecomposable projective-injective modules of $\mathcal{O}_{0}^{\mathfrak{p}}$. By a standard argument using the parabolic version of Soergel's functor $\mathbb{V}$ (see [Str03, Section 10]) it follows that this endomorphism algebra is isomorphic to the center of $\mathcal{O}_{0}^{\mathfrak{p}}$. Brundan [Bru08, Main Theorem] showed that this center is canonically isomorphic to $\mathbb{C}\left[x_{1}, \ldots, x_{n}\right] / I_{k}$, where $I_{k}$ is the ideal generated by

$$
\begin{aligned}
h_{r}\left(x_{i_{1}}, \ldots, x_{i_{m}}\right) & \text { for all } & 1 \leq m \leq n-k, & r>k, \\
h_{r}\left(x_{i_{1}}, \ldots, x_{i_{m}}\right) & \text { for all } & n-k+1 \leq m \leq n, & r>n-m .
\end{aligned}
$$

Notice that this result builds on a conjecture of Khovanov [Kho04, Conjecture 3] (proved in [Bru08, Main Theorem], [Str09, Theorem 1]) that the center of $\mathcal{O}_{0}^{\mathfrak{p}}$ agrees with the cohomology ring of a Springer fiber. Under this identification, the presentation (6.23) can be deduced from Tanisaki presentation Tan82 of the cohomology 
of the Springer fiber. Using (2.3) one can easily prove that the polynomials (6.23) generate the same ideal as (6.22).

For $\mathcal{E}_{k}$, by Proposition 6.10 we have $\operatorname{End}\left(\mathcal{E}_{k}\right) \cong \operatorname{End}_{A_{k+1} \otimes A_{k}^{\text {op }}}\left(\mathbf{E}_{k}\right)$. By Remark 5.37, it follows that

$$
\operatorname{End}\left(\mathcal{E}_{k}\right) \cong \operatorname{End}_{A_{k+1} \otimes A_{k}^{\mathrm{op}}}\left(\mathbf{E}_{k}\right) \cong \operatorname{End}_{A_{k} \otimes A_{k+1}^{\mathrm{op}}}\left(\mathbf{F}_{k}\right) \cong \operatorname{End}\left(\mathcal{F}_{k}\right)
$$

The middle isomorphism can be explained as follows: $\mathbf{E}_{k}$ and $\mathbf{F}_{k}$ have the same underlying vector space $V$; since the action of $A_{k+1} \otimes A_{k}^{\mathrm{op}}$ on $\mathbf{E}_{k}$ is just the action of $A_{k} \otimes A_{k+1}^{\text {op }}$ on $\mathbf{F}_{k}$ twisted (see Remark [5.37), a $\mathbb{C}$-linear endomorphism of $V$ is $A_{k+1} \otimes A_{k}^{\text {op }}$-equivariant (i.e., it is an endomorphism of $\mathbf{E}_{k}$ as an $\left(A_{k+1}, A_{k}\right)$ bimodule) exactly when it is $A_{k} \otimes A_{k+1}^{\text {op }}$-equivariant (i.e., it is an endomorphism of $\mathbf{F}_{k}$ as an $\left(A_{k}, A_{k+1}\right)$-bimodule).

The fact that the functors $\mathcal{E}_{k}$ and $\mathcal{F}_{k}$ are indecomposable follows since $\operatorname{End}\left(\mathcal{E}_{k}\right) \cong$ $\operatorname{End}\left(\mathcal{F}_{k}\right)$ is a graded local ring.

\section{ACKNOWLEDGMENTS}

The present paper is part of the author's Ph.D. thesis. The author would like to thank his advisor, Catharina Stroppel, for her help and support. The author would also like to thank an anonymous referee for many valuable comments and suggestions.

\section{REFERENCES}

[AM11] Troels Agerholm and Volodymyr Mazorchuk, On selfadjoint functors satisfying polynomial relations, J. Algebra 330 (2011), 448-467, DOI 10.1016/j.jalgebra.2011.01.004. MR2774639 (2012e:16041)

[Bac01] Erik Backelin, The Hom-spaces between projective functors, Represent. Theory 5 (2001), 267-283 (electronic), DOI 10.1090/S1088-4165-01-00099-1. MR1857082 (2002f:17007)

[BG80] J. N. Bernstein and S. I. Gel'fand, Tensor products of finite- and infinite-dimensional representations of semisimple Lie algebras, Compositio Math. 41 (1980), no. 2, 245-285. MR581584 (82c:17003)

[BGG76] I. N. Bernšteĭn, I. M. Gel'fand, and S. I. Gel'fand, A certain category of g-modules (Russian), Funkcional. Anal. i Priložen. 10 (1976), no. 2, 1-8. MR0407097 (53 \#10880)

[BGS96] Alexander Beilinson, Victor Ginzburg, and Wolfgang Soergel, Koszul duality patterns in representation theory, J. Amer. Math. Soc. 9 (1996), no. 2, 473-527, DOI 10.1090/S08940347-96-00192-0. MR1322847 (96k:17010)

[BJS93] Sara C. Billey, William Jockusch, and Richard P. Stanley, Some combinatorial properties of Schubert polynomials, J. Algebraic Combin. 2 (1993), no. 4, 345-374, DOI 10.1023/A:1022419800503. MR 1241505 (94m:05197)

[Bru08] Jonathan Brundan, Symmetric functions, parabolic category $\mathcal{O}$, and the Springer fiber, Duke Math. J. 143 (2008), no. 1, 41-79, DOI 10.1215/00127094-2008-015. MR2414744 (2009h:17007)

[BS10] Jonathan Brundan and Catharina Stroppel, Highest weight categories arising from Khovanov's diagram algebra. II. Koszulity, Transform. Groups 15 (2010), no. 1, 1-45, DOI 10.1007/s00031-010-9079-4. MR2600694 (2011b:17014)

[BS11a] Jonathan Brundan and Catharina Stroppel, Highest weight categories arising from Khovanov's diagram algebra I: cellularity (English, with English and Russian summaries), Mosc. Math. J. 11 (2011), no. 4, 685-722, 821-822. MR.2918294

[BS11b] Jonathan Brundan and Catharina Stroppel, Highest weight categories arising from Khovanov's diagram algebra III: category $\mathcal{O}$, Represent. Theory 15 (2011), 170-243, DOI 10.1090/S1088-4165-2011-00389-7. MR2781018 (2012b:17016)

[BS12a] Jonathan Brundan and Catharina Stroppel, Gradings on walled Brauer algebras and Khovanov's arc algebra, Adv. Math. 231 (2012), no. 2, 709-773, DOI 10.1016/j.aim.2012.05.016. MR.2955190 
[BS12b] Jonathan Brundan and Catharina Stroppel, Highest weight categories arising from Khovanov's diagram algebra IV: the general linear supergroup, J. Eur. Math. Soc. (JEMS) 14 (2012), no. 2, 373-419, DOI 10.4171/JEMS/306. MR2881300 (2012m:17009)

[CLO07] David Cox, John Little, and Donal O'Shea, Ideals, varieties, and algorithms, An introduction to computational algebraic geometry and commutative algebra, 3rd ed., Undergraduate Texts in Mathematics, Springer, New York, 2007. MR2290010 (2007h:13036)

[Dem73] Michel Demazure, Invariants symétriques entiers des groupes de Weyl et torsion (French), Invent. Math. 21 (1973), 287-301. MR.0342522 (49 \#7268)

[DM07] Yuriy Drozd and Volodymyr Mazorchuk, Koszul duality for extension algebras of standard modules, J. Pure Appl. Algebra 211 (2007), no. 2, 484-496, DOI 10.1016/j.jpaa.2007.01.014. MR2341265 (2008j:16083)

[Du05] Jie Du, Robinson-Schensted algorithm and Vogan equivalence, J. Combin. Theory Ser. A 112 (2005), no. 1, 165-172, DOI 10.1016/j.jcta.2005.01.008. MR2167481|(2006m:05253)

[EK09] Ben Elias and Mikhail Khovanov, Diagrammatics for Soergel categories, Int. J. Math. Math. Sci. (2010), Art. ID 978635, 58. MR3095655

[EW12] Ben Elias and Geordie Williamson, The Hodge theory of Soergel bimodules, Ann. of Math. (2) 180 (2014), no. 3, 1089-1136, DOI 10.4007/annals.2014.180.3.6. MR 3245013

[FK97] Igor B. Frenkel and Mikhail G. Khovanov, Canonical bases in tensor products and graphical calculus for $U_{q}\left(\mathfrak{s l}_{2}\right)$, Duke Math. J. 87 (1997), no. 3, 409-480, DOI 10.1215/S00127094-97-08715-9. MR1446615 (99a:17019)

[FKM02] V. Futorny, S. König, and V. Mazorchuk, Categories of induced modules and standardly stratified algebras, Algebr. Represent. Theory 5 (2002), no. 3, 259-276, DOI 10.1023/A:1016579318115. MR.1921761 (2003g:17005)

[Fri07] Anders Frisk, Dlab's theorem and tilting modules for stratified algebras, J. Algebra 314 (2007), no. 2, 507-537, DOI 10.1016/j.jalgebra.2006.08.041. MR2344576 (2009b:16028)

[Ful97] William Fulton, Young tableaux, With applications to representation theory and geometry, London Mathematical Society Student Texts, vol. 35, Cambridge University Press, Cambridge, 1997. MR1464693 (99f:05119)

[GL96] J. J. Graham and G. I. Lehrer, Cellular algebras, Invent. Math. 123 (1996), no. 1, 1-34, DOI 10.1007/BF01232365. MR1376244 (97h:20016)

[GR02] V. Gasharov and V. Reiner, Cohomology of smooth Schubert varieties in partial flag manifolds, J. London Math. Soc. (2) 66 (2002), no. 3, 550-562, DOI 10.1112/S0024610702003605. MR1934291 (2003i:14064)

[HM10] Jun $\mathrm{Hu}$ and Andrew Mathas, Graded cellular bases for the cyclotomic KhovanovLauda-Rouquier algebras of type A, Adv. Math. 225 (2010), no. 2, 598-642, DOI 10.1016/j.aim.2010.03.002. MR.2671176 (2011g:20006)

[Hum08] James E. Humphreys, Representations of semisimple Lie algebras in the BGG category $\mathcal{O}$, Graduate Studies in Mathematics, vol. 94, American Mathematical Society, Providence, RI, 2008. MR2428237 (2009f:17013)

[Kho00] Mikhail Khovanov, A categorification of the Jones polynomial, Duke Math. J. 101 (2000), no. 3, 359-426, DOI 10.1215/S0012-7094-00-10131-7. MR.1740682(2002j:57025)

[Kho04] Mikhail Khovanov, Crossingless matchings and the cohomology of $(n, n)$ Springer varieties, Commun. Contemp. Math. 6 (2004), no. 4, 561-577, DOI 10.1142/S0219199704001471. MR2078414 (2005g:14090)

[KL79] David Kazhdan and George Lusztig, Representations of Coxeter groups and Hecke algebras, Invent. Math. 53 (1979), no. 2, 165-184, DOI 10.1007/BF01390031. MR560412 (81j:20066)

[KL80] David Kazhdan and George Lusztig, Schubert varieties and Poincaré duality, Geometry of the Laplace operator (Proc. Sympos. Pure Math., Univ. Hawaii, Honolulu, Hawaii, 1979), Proc. Sympos. Pure Math., XXXVI, Amer. Math. Soc., Providence, R.I., 1980, pp. 185-203. MR573434 (84g:14054)

[Knu73] Donald E. Knuth, The art of computer programming. Volume 3, Sorting and searching; Addison-Wesley Series in Computer Science and Information Processing, AddisonWesley Publishing Co., Reading, Mass.-London-Don Mills, Ont., 1973. MR0445948 (56 \#4281)

[Mac91] I. G. Macdonald, Schubert polynomials, Surveys in combinatorics, 1991 (Guildford, 1991), London Math. Soc. Lecture Note Ser., vol. 166, Cambridge Univ. Press, Cambridge, 1991, pp. 73-99. MR1161461(93d:05159) 
[Maz04] Volodymyr Mazorchuk, Stratified algebras arising in Lie theory, Representations of finite dimensional algebras and related topics in Lie theory and geometry, Fields Inst. Commun., vol. 40, Amer. Math. Soc., Providence, RI, 2004, pp. 245-260. MR2057398 (2005b:17013)

[MS08] Volodymyr Mazorchuk and Catharina Stroppel, Projective-injective modules, Serre functors and symmetric algebras, J. Reine Angew. Math. 616 (2008), 131-165, DOI 10.1515/CRELLE.2008.020. MR2369489(2009e:16027)

[Sar13] A. Sartori, Categorification of tensor powers of the vector representation of $U_{q}(\mathfrak{g l}(1 \mid 1))$, arXiv e-prints (2013), 1305.6162.

[Sar14] A. Sartori, Ph.D. thesis, Universität Bonn, 2014.

[Soe90] Wolfgang Soergel, Kategorie $\mathcal{O}$, perverse Garben und Moduln über den Koinvarianten zur Weylgruppe (German, with English summary), J. Amer. Math. Soc. 3 (1990), no. 2, 421-445, DOI 10.2307/1990960. MR1029692(91e:17007)

[Soe92] Wolfgang Soergel, The combinatorics of Harish-Chandra bimodules, J. Reine Angew. Math. 429 (1992), 49-74, DOI 10.1515/crll.1992.429.49. MR1173115 (94b:17011)

[Soe97] Wolfgang Soergel, Kazhdan-Lusztig-Polynome und eine Kombinatorik für Kipp-Moduln (German, with English summary), Represent. Theory 1 (1997), 37-68 (electronic), DOI 10.1090/S1088-4165-97-00006-X. MR.1445511 (99d:17023)

[Str03] Catharina Stroppel, Category $\mathcal{O}$ : quivers and endomorphism rings of projectives, Represent. Theory 7 (2003), 322-345 (electronic), DOI 10.1090/S1088-4165-03-00152-3. MR2017061 (2004h:17007)

[Str09] Catharina Stroppel, Parabolic category $\mathcal{O}$, perverse sheaves on Grassmannians, Springer fibres and Khovanov homology, Compos. Math. 145 (2009), no. 4, 954-992, DOI 10.1112/S0010437X09004035. MR2521250(2011a:17014)

[SW12] Catharina Stroppel and Ben Webster, 2-block Springer fibers: convolution algebras and coherent sheaves, Comment. Math. Helv. 87 (2012), no. 2, 477-520, DOI 10.4171/CMH/261. MR 2914857

[Tan82] Toshiyuki Tanisaki, Defining ideals of the closures of the conjugacy classes and representations of the Weyl groups, Tôhoku Math. J. (2) 34 (1982), no. 4, 575-585, DOI 10.2748/tmj/1178229158. MR685425 (84g:14049)

[Wil11] Geordie Williamson, Singular Soergel bimodules, Int. Math. Res. Not. IMRN 20 (2011), 4555-4632, DOI 10.1093/imrn/rnq263. MR2844932

Mathematisches Institut, Endenicher Allee 60, Universität Bonn, 53115 Bonn, GerMANY

Current address: Mathematisches Institut, Albert-Ludwigs-Universität Freiburg, Eckerstraße

1, 79104 Freiburg im Breisgau, Germany

E-mail address: antonio.sartori@math.uni-freiburg.de

$U R L:$ http://home.mathematik. uni-freiburg.de/asartori/ 